\title{
\#USGS
}

D.t.

Prepared in cooperation with the U.S. Fish and Wildifife Service

Assessing the Risks Posed by SARS-CoV-2 in and via Nortif American Bats-Decision Framing and Rapid Risk Assessment

Open-File Report 2020-1060

U.S. Department of the Interior

U.S. Geological Survey 
Cover. A single Myotis lucifugus (little brown bat; black nose) in a cluster of M. sodalis (Indiana bats; pink noses). Photo by Riley Bernard, University of Tennessee. 


\section{Assessing the Risks Posed by SARS-CoV-2 in and via North American Bats- Decision Framing and Rapid Risk Assessment}

By Michael C. Runge, Evan H. Campbell Grant, Jeremy T. H. Coleman, Jonathan D. Reichard, Samantha E. J. Gibbs, Paul M. Cryan, Kevin J. Olival, Daniel P. Walsh, David S. Blehert, M. Camille Hopkins, and Jonathan M. Sleeman

Prepared in cooperation with the U.S. Fish and Wildlife Service

Open-File Report 2020-1060 


\title{
U.S. Department of the Interior DAVID BERNHARDT, Secretary
}

\author{
U.S. Geological Survey \\ James F. Reilly II, Director
}

U.S. Geological Survey, Reston, Virginia: 2020

For more information on the USGS - the Federal source for science about the Earth, its natural and living resources, natural hazards, and the environment-visit https://www.usgs.gov or call 1-888-ASK-USGS.

For an overview of USGS information products, including maps, imagery, and publications, visit https://store.usgs.gov.

Any use of trade, firm, or product names is for descriptive purposes only and does not imply endorsement by the U.S. Government.

Although this information product, for the most part, is in the public domain, it also may contain copyrighted materials as noted in the text. Permission to reproduce copyrighted items must be secured from the copyright owner.

Suggested citation:

Runge, M.C., Grant, E.H.C., Coleman, J.T.H., Reichard, J.D., Gibbs, S.E.J., Cryan, P.M., Olival, K.J., Walsh, D.P., Blehert, D.S., Hopkins, M.C., and Sleeman, J.M., 2020, Assessing the risks posed by SARS-CoV-2 in and via North American bats-Decision framing and rapid risk assessment: U.S. Geological Survey Open-File Report 2020-1060, 43 p., https://doi.org/10.3133/ofr20201060. 


\section{Acknowledgments}

A guidance committee, composed of representatives from several State and Federal wildlife agencies, was instrumental in helping us frame the decision context for this risk assessment. We are grateful to Jenny Dickson of the Connecticut Department of Energy and Environmental Protection, Owen Boyle of the Wisconsin Department of Natural Resources, Colin Gillin of the Oregon Department of Fish and Wildlife, Carl Herzog of the New York State Department of Environmental Conservation, Richard Reynolds of the Virginia Department of Game and Inland Fisheries, Michelle Verant of the National Park Service, and Patrice Klein of the Forest Service.

The members of the expert panel were instrumental in helping us estimate the underlying parameters in this risk assessment. For their expertise, thoughtfulness, and patience, we thank Raina Plowright from Montana State University, DeeAnn Reeder from Bucknell University, Daniel Streicker from the University of Glasgow (United Kingdom), Jonathan Epstein from EcoHealth Alliance, Christine Kreuder Johnson from the University of California at Davis, Winifred Frick from Bat Conservation International, Jonathan Towner and Brian Amman from the U.S. Centers for Disease Control and Prevention, Alison Peel from Griffith University (Australia), Rebekah Kading from Colorado State University, Amy Gilbert from the U.S. Department of AgricultureAPHIS, Kevin Castle from Wildlife Veterinary Consulting LLC, and Kate Jones from University College London (UK).

Matthew Ferrari from the Pennsylvania State University and Jennifer Schimmel from Baystate Health Division of Infectious Diseases provided insights into the human epidemiology of SARSCoV-2. Justin Stevenson of RD Wildlife Management and Consulting provided additional context for wildlife control activities. We are grateful to LeAnn White, Katherine Richgels, and Karen Jenni of the U.S. Geological Survey, who carefully reviewed this report and offered suggestions that have improved it substantially.

The findings and conclusions in this article are those of the authors and do not necessarily represent the views of the U.S. Fish and Wildlife Service. 


\section{Contents}

Acknowledgments .............................................................................................................ii

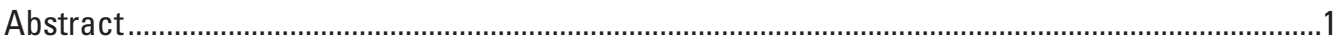

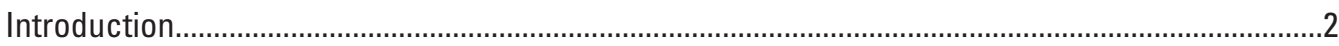

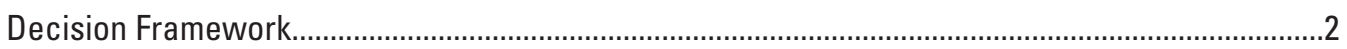

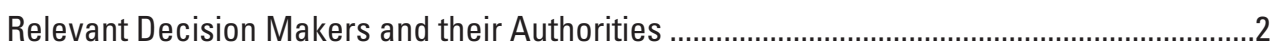

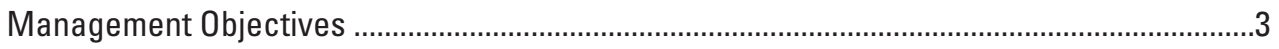

Potential Mitigation Measures ..............................................................................................

Causal Linkages between Actions and Objectives ....................................................................

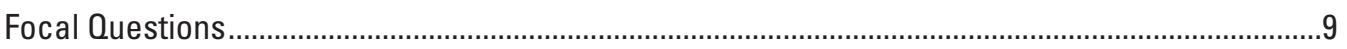

Model for the Direct Transmission Pathways ………….........................................................

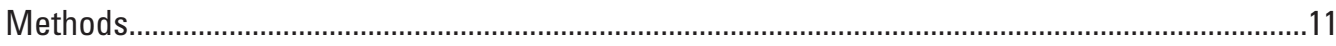

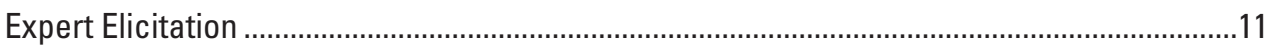

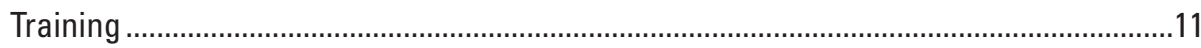

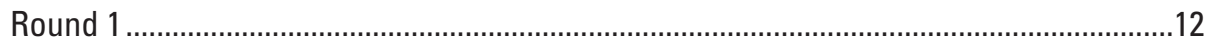

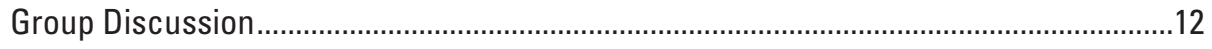

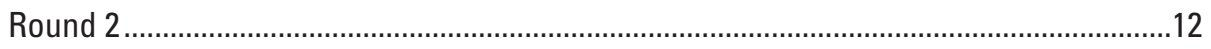

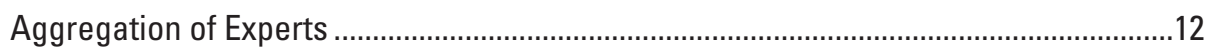

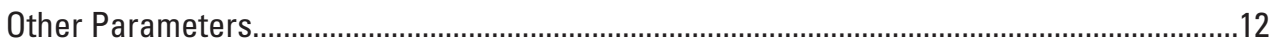

Bat Handling Data ............................................................................................12

Probability an Individual Human is Shedding SARS-CoV-2 …....................................12

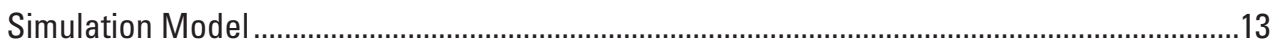

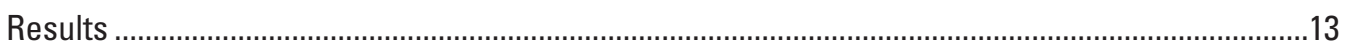

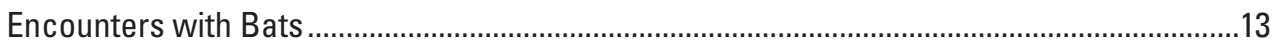

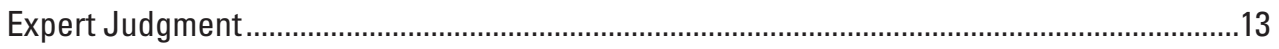

Probability a Worker is Shedding Virus .............................................................................

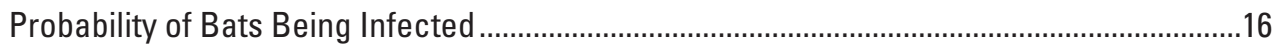

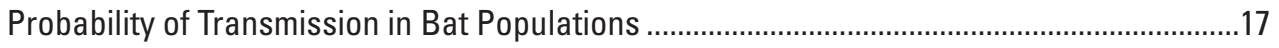

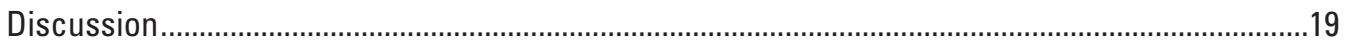

Summary of Results .....................................................................................................

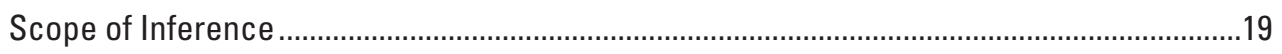

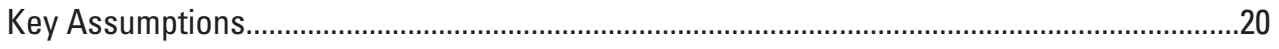

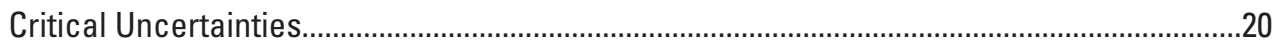

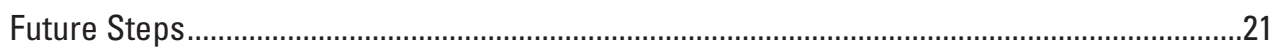

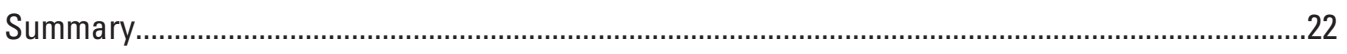

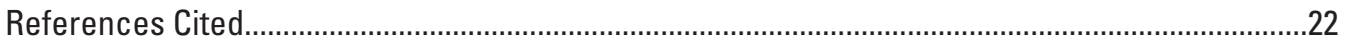

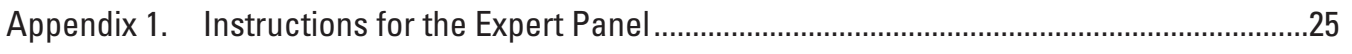

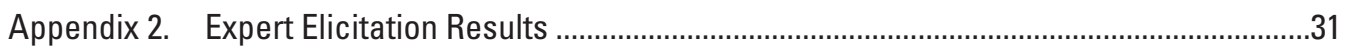




\section{Figures}

1. Influence diagram showing the causal linkages between actions and outcomes motivated by the risk of SARS-CoV-2 entering North American bat populations..

2. The part of the influence diagram that focuses on the routes of initial transmission of SARS-CoV-2 from humans to bats, with different levels of potential mitigation strategies

3. Graph showing aggregate probability distributions for the probability of exposure of bats from a SARS-CoV-2-positive research, survey, monitoring, and management worker, referenced in expert judgment questions 01,02 , and 03 .

4. Graph showing aggregate probability distributions for the effectiveness of proper use of personal protective equipment in reducing exposure of bats to SARS-CoV-2 by a research, survey, monitoring, and management worker, referenced in expert judgment questions 09,010 , and 011 , expressed as the percent reduction in exposure

5. Boxplot showing probability that a worker is shedding SARS-CoV-2 during an encounter with a bat

6. Boxplots of the number of bats per 1,000 exposed to and infected by virus from a SARS-CoV-2-positive worker, by transmission pathway, under status quo working conditions.

7. Boxplots of the number of bats per 1,000 exposed to and infected by SARS-CoV-2 from an average worker, by transmission pathway, without and with the proper use of personal protective equipment.

8. Boxplots of the number of bats per 1,000 exposed to and infected by SARS-CoV-2 from an average scientist in the RSM pathway, by encounter type, without and with proper use of personal protective equipment.

9. Boxplot of the basic reproduction number, $R_{0^{\prime}}$ for SARS-CoV-2 in little brown bats, as estimated by an expert panel

\section{Tables}

1. Number of bats encountered during the 2019 active bat season 


\title{
Conversion Factors
}

U.S. customary units to International System of Units

\begin{tabular}{|c|c|c|}
\hline Multiply & By & To obtain \\
\hline \multicolumn{3}{|c|}{ Length } \\
\hline inch (in.) & 2.54 & centimeter $(\mathrm{cm})$ \\
\hline inch (in.) & 25.4 & millimeter $(\mathrm{mm})$ \\
\hline foot (ft) & 0.3048 & meter (m) \\
\hline mile (mi) & 1.609 & kilometer $(\mathrm{km})$ \\
\hline mile, nautical (nmi) & 1.852 & kilometer $(\mathrm{km})$ \\
\hline yard (yd) & 0.9144 & meter (m) \\
\hline
\end{tabular}

\section{Abbreviations}

\author{
AFWA Association of Fish and Wildlife Agencies \\ CDC Centers for Disease Control and Prevention \\ CoV+ Actively shedding SARS-CoV-2 virus \\ DOI U.S. Department of the Interior \\ ESA U.S. Endangered Species Act, 16 U.S.C. \$1531 et seq. \\ FWS U.S. Fish and Wildlife Service \\ IACUC Institutional Animal Care and Use Committee \\ IDEA The Investigate-Discuss-Estimate-Aggregate Protocol \\ IQR Interquartile range \\ NGO Non-governmental organization \\ NPS National Park Service \\ OIE World Organisation for Animal Health (Office International des Epizooties) \\ PDF Probability density function \\ PPE Personal protective equipment \\ RSM Research, Survey, Monitoring, and Management \\ USFS U.S. Department of Agriculture, Forest Service \\ USGS U.S. Geological Survey \\ WC Wildlife Control \\ WCO Wildlife Control Officer \\ WR Wildlife Rehabilitation \\ WNS White-nose syndrome
}




\title{
Assessing the Risks Posed by SARS-CoV-2 in and via North American Bats-Decision Framing and Rapid Risk Assessment
}

\author{
By Michael C. Runge ${ }^{1}$, Evan H. Campbell Grant ${ }^{1}$, Jeremy T. H. Coleman², Jonathan D. Reichard², \\ Samantha E. J. Gibbs'ㄹ, Paul M. Cryan'1, Kevin J. Olival', Daniel P. Walsh', David S. Blehert', \\ M. Camille Hopkins', and Jonathan M. Sleeman' ${ }^{1}$
}

\section{Abstract}

The novel $\beta$-coronavirus, SARS-CoV-2, may pose a threat to North American bat populations if bats are exposed to the virus through interaction with humans, if the virus can subsequently infect bats and be transmitted among them, and if the virus causes morbidity or mortality in bats. Further, if SARS-CoV-2 became established in bat populations, it could possibly serve as a source for new infection in humans, domesticated animals, or other wild animals. Wildlife management agencies in the United States are concerned about these potential risks and have begun to issue guidance regarding work that brings humans into contact with bats, but decision making is difficult because of the high degree of uncertainty about many of the relevant processes that could lead to virus transmission and establishment. The risk assessment described in this report was undertaken to provide management agencies with an understanding of the likelihood that the various steps in the causal pathways would lead to SARS-CoV-2 infection of North American bats from people. This assessment focused on the active season for bats in the temperate zone of North America (April 15 through November 15), and used Myotis lucifugus (little brown bats) as a surrogate species. At the time of this work (April 2020), no empirical data about the effects of SARS-CoV-2 on North American bats were available, so a formal process of expert judgment was used to elicit estimates of the underlying parameters. Twelve experts in bat ecology, epidemiology, virology, and wildlife disease from the United States, United Kingdom, and Australia participated in the elicitation. A Monte Carlo simulation model was used to integrate the parameter estimates elicited from the experts and to predict the likelihood of exposure and infection in bats through a

\footnotetext{
'U.S. Geological Survey.

${ }^{2}$ U.S. Fish and Wildlife Service.

${ }^{3}$ EcoHealth Alliance.
}

series of transmission pathways, with particular attention to capturing uncertainty in the predictions.

Given the current state of knowledge as expressed by the expert panel, the results of this assessment indicate that there is a non-negligible risk of transmission of SARS-CoV-2 from humans to bats. For example, if a research scientist were shedding SARS-CoV-2 virus while handling bats under the field protocols used in North America prior to the COVID-19 pandemic, the risk model indicates that 50 percent (uncertainty, 15-84 percent) of those bats could be exposed to virus, and 17 percent (uncertainty, 3-51 percent) could become infected. Use of personal protective equipment, especially a respirator, is expected to reduce the exposure risk. The expert panel estimated that exposure risk from research scientists could be reduced 94-96 percent (uncertainty, 86-99 percent) through proper use of appropriate N95 respirators (a type of mechanical filter worn over the nose and mouth), dedicated clothing (such as Tyvek coveralls), and gloves. Should any North American bats become infected with SARS-CoV-2, the expert panel estimated that there is an approximately 33-percent chance the virus could spread within a bat population.

This study, conducted by the U.S. Geological Survey in cooperation with the U.S. Fish and Wildlife Service, identified several critical uncertainties that could affect the estimate of risks associated with SARS-CoV-2 entering bat populationsnotably, the underlying probability that a human would be shedding virus while working with bats, the likelihood of the virus replicating in bat tissue, and the likelihood of transmission of the virus within bat populations. Ongoing empirical work during May-October 2020 may shed light on these issues. Follow-up work is needed to better understand the probability of transmission of SARS-CoV-2 to bats from the general public; the manner in which the probabilities of exposure, infection, and transmission would differ during hibernation compared to the breeding season; and the likelihood of important effects, like morbidity and mortality in bats, the possibility of zoonosis from a North American bat reservoir, and effects of and on other wildlife. 


\section{Introduction}

The novel $\beta$-coronavirus, SARS-CoV-2, that has caused a pandemic disease (COVID-19) in humans arose from a mammalian host, possibly an Old World bat in the family Rhinolophidae. The closest known virus discovered in wildlife was found in a Rhinolophus affinis (horseshoe bat) from Yunnan province in China (Zhou and others, 2020b), although the similarity is not an exact match. No SARS-related $\beta$-coronaviruses have yet been identified in New World bats, but a different type of $\beta$-coronavirus has been identified in New World species of bats from Mexico (Anthony and others, 2013; Anthony and others, 2017; Góes and others, 2016). This raises an important question about whether North and South American bats could be vulnerable to infection with SARS$\mathrm{CoV}-2$ via contact with humans, which in turn raises questions about whether there could be reciprocal spread to humans via a bat reservoir. This inquiry was designed to be a rapid assessment of the risk of transmission of SARS-CoV-2 from humans to North American bats, the management contexts in which this risk might be relevant, and possible mitigation actions that may be implemented by those who come into contact with bats or their habitats. The structure of this study could also serve as a model to rapidly assess the risk to bats in other geographic regions (for example, Europe or Latin America) or the risk to other wildlife taxa of concern (for example, felids and mustelids which may be susceptible to SARS-CoV-2; Shi and others, 2020).

The purpose of this report is to describe the risk assessment conducted by the U.S. Geological Survey, in cooperation with the U.S. Fish and Wildlife Service, to evaluate the potential for transmission of SARS-CoV-2 from humans to bats. This assessment focuses on potential activities undertaken by research scientists, wildlife rehabilitators, and wildlife control operators in North America during the summer field season (April 15 to November 15, 2020), with and without new protocols for such work.

\section{Decision Framework}

In late March 2020, State, Federal, and tribal wildlife management agencies in the United States began expressing concern about the possible transmission of SARS-CoV-2 from humans to bats and requested that the U.S. Geological Survey (USGS) lead a risk assessment that could inform their decision making. Prior to designing the risk assessment, the authors worked with a guidance committee composed of representatives from State and Federal wildlife management agencies (see Acknowledgments) to frame the decision context in which risk assessment would be used. We recognized that the motivation, statutory requirements, and authority to address the problem may stem from human health and wildlife conservation interests and needs, and that decisions involve a mixture of conservation and human health objectives, where tradeoffs are likely to occur. The construction of the decision framework was instrumental in informing the focus and structure of the risk assessment. The decision framework constructed in consultation with the representatives from decision-making agencies is described below.

\section{Relevant Decision Makers and their Authorities}

In the United States, there are many decision makers with authority to make decisions that affect bats and the interactions of bats and people. For most terrestrial mammal species that are not listed under the U.S. Endangered Species Act (ESA; 16 U.S.C. $\S 1531$ et seq.) and are not on Federal land, the State wildlife agencies have management jurisdiction. The status of bats under current State laws and regulations differs from State to State (O'Shea and others, 2018), but existing statutory and regulatory authorities generally involve several types of activity. First, States have authority to direct the activities of their own staff, such as conducting bat surveys or habitat and population management. Second, States permit the work of wildlife rehabilitators and can prescribe conditions of that work. Third, States permit nuisance wildlife control operators who perform such activities as removing bats from human dwellings. Fourth, States provide permits for a variety of research activities by scientists and environmental consultants. Fifth, States sometimes collaborate with educational institutions that may keep captive bats for purposes of exhibition. Sixth, States often require permits or registration for private citizens or groups who wish to hold bats. Seventh, State wildlife agencies, in conjunction with many partner agencies, often undertake public communication about the benefits of wildlife and healthy ways for humans to interact with wildlife.

Several U.S. Department of the Interior (DOI) agencies have management responsibilities for bats. The U.S. Fish and Wildlife Service (FWS) has authority under the ESA for any listed bat species; this authority includes permitting the activities of other Federal agencies that may affect listed species. The FWS Office of Law Enforcement is responsible for managing the importation of wildlife into the country. (The Centers for Disease Control and Prevention (CDC) also require permits for importation of bats). The FWS Wildlife and Sport Fish Restoration Program is a major funding source for State agencies' bat management efforts. The FWS supports the National White-Nose Syndrome Program, which provides funding for research, conservation, and monitoring of bats, and issues guidance to partners on matters related to white-nose syndrome (WNS). The FWS, through the National Wildlife Refuge System, manages land, wildlife, staff, and public access at Refuges, some of which provide habitat for bats. Refuge staff conduct research and monitoring activities. Refuges also issue special use permits for outside scientists to conduct research. Similarly, the National Park Service (NPS) has authority over the activities that occur within NPS 
units. These activities include research and management, which involve contact with bats by park staff or cooperators; cave tours and bat viewing opportunities for the public; and permits for recreational caving by individuals or groups. The FWS Refuge System and NPS have extensive communication efforts aimed at educating the public about wildlife, including bats.

Within the U.S. Department of Agriculture, the U.S. Forest Service (USFS) has research and management responsibilities for bats on its Federal lands, often in coordination with other Federal and State partners. Similar to DOI agencies, the USFS manages land, sustains habitat for native fish and wildlife, and provides public access for recreational activities, including use of caves and mines. USFS scientists conduct research and monitoring activities. The USFS issues permits for outside scientists to conduct research on its lands. The National Forest System engages in public outreach and education about wildlife, especially bats.

Many agencies and institutions, including the USGS, FWS, NPS, USFS, universities, and non-governmental organizations, conduct scientific studies on bats. Under the Animal Welfare Act (7 U.S.C. §2131 et seq.) and other Federal and State laws and policies, researchers and agencies are required to consider the welfare of the animals being studied or used for educational or teaching purposes in captivity or the wild. Institutional Animal Care and Use Committees (IACUC) are responsible for review and approval of protocols involving animals used in research or teaching. IACUC review ensures that the welfare of animals is taken into consideration and that all approved activities include appropriate use of animals. Additionally, these entities often conduct internal reviews of scientific research study plans. IACUC and other research plan review mechanisms represent an additional tool with which agencies can manage the activities of their staff that involve interactions with animals.

Public health agencies at Federal, State, and county levels take action to benefit human health, and while they do not directly manage bats, they do play a role in the interactions humans have with bats. Because of the risk of rabies virus transmission, many interactions that members of the general public have with bats are reported to county health agencies, which help to manage the health risk of such exposure. In turn, county health agencies often report rabies statistics to State and Federal public health agencies (for example, CDC), enabling broader understanding of seasonal and geographic patterns and trends in the types of bats and other wildlife coming into contact with the public at a national scale (Pieracci and others, 2020).

Finally, zoos and wildlife parks frequently have captive populations of bats, including species native to North America as well as species from other parts of the world, with which North American bats may not naturally come into contact. Zoos and wildlife parks can control the proximity of humans to captive bats and the way the bats are handled by staff.

\section{Management Objectives}

Each agency with jurisdiction that affects the interactions of bats and humans has its own purposes, as derived from its enabling legislation, mission, or stakeholder input. We worked with representatives from State and Federal wildlife management agencies (see Acknowledgments) to develop a set of long-term outcomes ("fundamental objectives") sought by these agencies through decisions related to bats and SARS-CoV-2. We recognize that some of these objectives may conflict with each other; indeed, that is what makes decisions difficult. By clearly articulating the set of objectives that are important to decision makers, the scientific assessments that are needed to inform difficult deliberations about appropriate and necessary mitigation actions can be better identified. Through discussions with these representatives, 10 objectives were identified. (The order of presentation of these objectives is not meant to imply anything about their relative importance.)

1. Minimize the morbidity and mortality of wild North American bats resulting from infection with SARS$\mathrm{CoV}-2$ or from management actions meant to mitigate transmission. If SARS-CoV-2 is introduced and transmitted to a naïve population of bats, it is possible the novel infection could lead to disease or death. In addition, some management actions meant to reduce disease transmission to bats could directly or indirectly cause mortality. Because many of these bat populations are already threatened by WNS and other stressors, any additional sources of mortality could affect long-term conservation.

2. Minimize the risk of SARS-CoV-2 becoming endemic in any North American bat population through sustained bat-to-bat transmission. We want to avoid anthropogenic establishment of a new endemic disease in bat populations for several reasons. Fundamentally, any anthropogenic change to the ecosystem outside the course of natural events is to be avoided. We are also concerned about this objective as a means to other objectives because a reservoir of SARS-CoV-2 in bats could lead to a reduction in the long-term conservation of bats (Objective 1), a risk to public health (Objective 3), or a risk to the health of other wildlife taxa or domesticated animals (Objective 5).

3. Minimize the risk of new SARS-CoV-2 cases in humans via transmission from North American bats. The longterm aim of public health agencies and other organizations will be to minimize the incidence and transmission of SARS-CoV-2 in humans. However, if a reservoir of SARS-CoV-2 becomes established in North American bats, it could represent a source for new exposure and infection; worse, if such a reservoir provides an opportu- 
nity for evolution or recombination of the virus, the new viral strains could evade existing immune responses or reduce the efficacy of vaccines under development.

4. Minimize the indirect effect on human health from actions designed to mitigate SARS-CoV-2 transmission to bat populations. Particularly at this moment, when the equipment needed to manage the COVID-19 pandemic is in short supply, any use of such equipment (such as personal protective equipment; PPE) for bat-related activities may undermine public health efforts. The supply of PPE for human needs is expected to increase in the near future as manufacturing ramps up, decreasing the gap between demand and supply. Therefore, this may not be a limitation once the human health demand is satisfied.

5. Minimize the risk of SARS-CoV-2 infection in other North American wildlife or domesticated animal populations through a reservoir in North American bats. Other species of mammals and other taxa are known to be susceptible to $\beta$-coronaviruses, specifically SARSrelated viruses. If SARS-CoV-2 becomes established in bat populations, it could possibly spill over into other susceptible wild and domesticated animals.

6. Maintain or maximize the ability of wildlife control operators and wildlife rehabilitators to carry out their functions for the benefit of humans and wildlife. The activities undertaken by wildlife control operators are necessary tools for managing conflict between humans and wildlife; these activities (like humane removal of bats from human dwellings and prevention of ingress) are important for human health (for example, minimizing rabies exposure) as well as for bat conservation. Likewise, the activities undertaken by wildlife rehabilitators may have a positive effect on wildlife, as well as a positive effect on public attitudes toward wildlife.

7. Maintain or maximize recreational activities, such as cave tours, recreational caving, and other activities that occur in bat habitat. Humans derive benefit from outdoor recreational activities; indeed, refuges, parks, and national forests have an important purpose in providing such opportunities.

8. Maximize the opportunities for scientific research on bats and within bat habitat. Research on bats and their habitats contributes to many facets of primary knowledge about the natural world. Conservation measures for other threats to bats, including WNS, benefit from on-going research. Additionally, the status of listed and candidate bat species requires a periodic assessment of population sizes. The fields of geology, hydrology, entomology, and numerous others benefit from on-going research that may overlap with bats and their habitats.
9. Maximize public appreciation for bats and their conservation. Past zoonotic diseases (such as the 2003-10 highly pathogenic avian influenza outbreak and the 2003 SARS outbreak) have created negative public responses to wildlife (wild birds and wild bats, respectively). The risk of SARS-CoV-2 in bats and the response to it could undermine recent gains in public appreciation for bats and bat conservation.

10. Maximize the ability to manage and conserve bat populations. Many of the agencies mentioned above have active programs to conserve bat populations. These programs sometimes require staff to handle or be in proximity to bats. Objectives 6 and 8 also contribute to the long-term conservation of North American bats.

\section{Potential Mitigation Measures}

The central causal chain that was motivating concern from State, Federal, and tribal wildlife agencies has three steps: the possible transmission of SARS-CoV-2 from humans to bats; sustained bat-to-bat transmission of SARS-CoV-2; and subsequent effects, for instance, of transmission from bats back to humans or to other wildlife. The representatives from State and Federal wildlife agencies that guided this work expressed an urgent need to identify actions they could take to interrupt this potential chain of events. We worked with the representatives to identify the types of actions within their jurisdiction that could be employed to minimize the risks associated with SARS-CoV-2 and achieve the objectives described above. Each of the decision-making bodies has a different set of management actions under its jurisdiction, and we did not try to match particular actions with specific agencies. Instead, we worked with them to describe the types of actions that could be taken in an attempt to achieve the objectives described earlier. It is worth noting that these actions are not mutually exclusive; indeed, a full strategy may involve deploying these actions in combination.

Federal and State agencies have a variety of mechanisms by which they may implement mitigation strategies. These mechanisms may come in the form of regulations, guidance, directives, conditions of funding, or permission.

- Various agencies have authority to issue permits (for example, for the take or harassment of Federally Threatened or Endangered bat species, to conduct research on bats, for research activities on National Wildlife Refuges and National Forests, for wildlife holding, for scientific take, for school programs and citizen scientists, for wildlife control operators, and to operate wildlife rehabilitation centers). Agencies may reject or rescind permission for activities that involve handling or proximity to bats or bat habitats. Additionally, permission may be granted so long as a permittee takes a set of risk-mitigation actions (for example, 
from the list below). Permittees include university, tribal, Federal, and State agency researchers; environmental consultants; wildlife rehabilitators; and wildlife control operators.

- Multiple agencies (USGS, FWS, NPS, USFS) have Institutional Animal Care and Use Committees, institutional biosafety committees, and other research approval processes (for example, study plan review and approval) or benefit from these committees housed with non-governmental institutions (universities, professional societies, non-governmental organizations); the committees may compel researchers to comply with risk-mitigation measures as they apply to animal welfare concerns. These committees may suspend animal research that has received prior approval until risk mitigation measures germane to animal welfare are adopted.

- Agencies (for example, USGS science centers and Cooperative Research Units, FWS-WNS National Program, NPS visitor contact, and USFS) may issue guidance outlining voluntary measures, which differs from a permit in that the guidance is not compulsory. Agencies may issue guidance and provide training of wildlife rehabilitation and nuisance animal control entities to promote the adherence to best practices.

Potential mitigation actions may reduce the frequency of contact or the probability of transmission of SARS-CoV-2 from infected humans to susceptible bats or change the behavior of individuals interacting with bat populations.

1. The use of PPE, including appropriate N95 respirators or other face masks or shields, eye protection, latex or nitrile gloves, or dedicated clothing (for example, coveralls, Tyvek) to minimize exposure of bats from COVID-19-infected individuals may be required (for example, via agency permit, occupational safety and health programs, or IACUC).

2. Decontamination protocols, such as those provided by the National WNS Response Team to reduce the threat posed by the fungal pathogen Pseudogymnoascus destructans, may include best-practice protocols for disinfection of persons and equipment prior to and after handling bats or interacting with bat habitats.

3. Various agencies (for example, States, NPS, Association of Fish and Wildlife Agencies [AFWA], FWS-WNS National program, USFS) have avenues for public outreach, which are already in use to improve public understanding and tolerance of bats, and can be used to encourage the public to engage in behavior and adopt protective measures (including the use of PPE and distancing between humans and bats) to reduce the risk of transmission of SARS-CoV-2 to bats. In some circumstances, this public outreach is coordinated with State and local public health agencies or the CDC.
4. Land managers, including NPS, FWS, USFS, States, and tribes may suspend or limit access to bat populations or habitats. This may involve suspending group tours to caves and access to National Wildlife Refuges or requiring or encouraging distancing from bats. This may also involve directing permittees and agency personnel to delay or suspend some kinds of (non-essential) research.

5. Nuisance animal control activities (undertaken by wildlife control operators [WCO], which may include such activities as capturing individual bats in a home, trapping and transporting bats in an attic, and installing an exclusion device to restrict bat access to human dwellings) could be legally limited to currently established best management practices, which prevent the unnecessary human-bat interactions that are characteristic of less effective approaches. WCOs could be advised or required to not release hand-captured bats.

6. Wildlife rehabilitators may be instructed to avoid accepting bats for rehabilitation.

7. Wildlife rehabilitators may be instructed to not release captive bats or to suspend wildlife rehabilitation of bats. As appropriate testing becomes available, bats might only be released after testing negative for coronavirus.

8. Agencies may issue guidance or directives governing conditions for workers coming into contact with bats, such as a minimum amount of time (for example, 2 weeks) after any potential exposure to SARS-CoV-2, a negative test for active coronavirus infection, a positive serological test, or vaccination to SARS-CoV-2 (when it is available).

9. Agencies, universities, and non-governmental organizations (NGOs) may begin research projects on the risks of SARS-CoV-2 transmission between humans and bats and the effectiveness of potential mitigation options.

10. In the long term, if SARS-CoV-2 does become established in North American bat populations, there might be mitigation measures designed to prevent the virus from moving back to humans, wildlife, or domesticated animals. These may differ from actions identified above.

\section{Causal Linkages between Actions and Objectives}

An influence diagram is a graphical representation of a system. The influence diagram in figure 1 describes the causal chains - pathways within the human-bat system - that link the mitigation actions with the desired outcomes. Each arrow represents a process, which is governed by a parameter that describes the effect of an action on an outcome. Figure 1 shows the proposed causal linkages between the potential mitigation actions (orange rectangles) and the long-term objectives 
(blue hexagons) described above. Key system states are represented as nodes in the diagram (green ovals).

The central causal logic that is motivating discussions about SARS-CoV-2 in North American bats can be described with three phases: (1) direct or indirect transmission of SARS-CoV-2 from a human to individual wild bats (dashed portion of fig. 1); (2) following this initial transmission, sustained bat-to-bat transmission of SARS-CoV-2, resulting in endemic disease within one or more bat populations (arrow labelled "Sustained bat-to-bat transmission" in fig. 1); and (3) ensuing consequences of endemic SARS-CoV-2 in bats, such as transmission to humans, other wildlife, or domesticated animals (arrows to hexagons in fig. 1). The influence diagram captures the expectation that many of the actions designed to mitigate the risks posed by SARS-CoV-2 have consequences to other important outcomes. The choice of mitigation measures may require decision makers to balance trade-offs among multiple objectives.

Some of the parameters underlying the central steps in figure 1 are transmission parameters (between humans and bats, among bats, between bats and other species, among locations, and between bats and humans) and bat mortality parameters. Mitigation actions are expected to affect the transmission rates, which may subsequently affect the bat mortality rates; we did not consider any actions that address bat mortality rates directly. We recognize that there is likely some background level of transmission (through the general public node, and possibly through a feral/domestic cat node) that may not be affected by any of the mitigation actions considered.

To estimate the consequences of a set of potential mitigation strategies (combinations of the 10 actions described above), we need estimates of parameter values for many of the arrows in the influence diagram (fig. 1). Given the novelty of SARS-CoV-2, there is a lack of robust scientific information for many of these parameters at this time. Values for the parameters may be borrowed from similar systems reported in the scientific literature, but the manner in which these are applied to North American bat populations requires expert judgment.

This influence diagram helps to identify the key parameters for which we require quantitative estimates to calculate the resulting risk, given the selection of a mitigation strategy (that is, one or more actions). In this first phase, we primarily focus on estimating the risk of transmission to bat populations via direct contact with humans (fig. 2), particularly during the time of year when bats are most active and may be in contact with the most humans, which are in the midst of the COVID19 pandemic (2020). If it is possible to prevent the initial transmission, then all the later steps in the causal pathway are blocked. Further, even if transmission cannot be entirely prevented (for example, because of other pathways of transmission), the reduction in the magnitude of transmission may decrease the risks associated with later steps. We recognized the need to estimate the probability of sustained bat-to-bat transmission. This step is critical to many of the later consequences, so if that risk is very low, the need for mitigation might be less urgent.

The dynamics of initial transmission, although a portion of the larger picture, are nevertheless sufficiently complicated for the first step of our risk analysis. Key factors to consider include potential differences among bat species, regions, and initial population status (including population size and the occurrence of Pseudogymnoascus destructans and WNS); differences among the routes of transmission; and differences among the mitigation actions (for example, using PPE in research settings may include appropriate N95 respirators or less protective masks). 


\section{Mitigation actions}

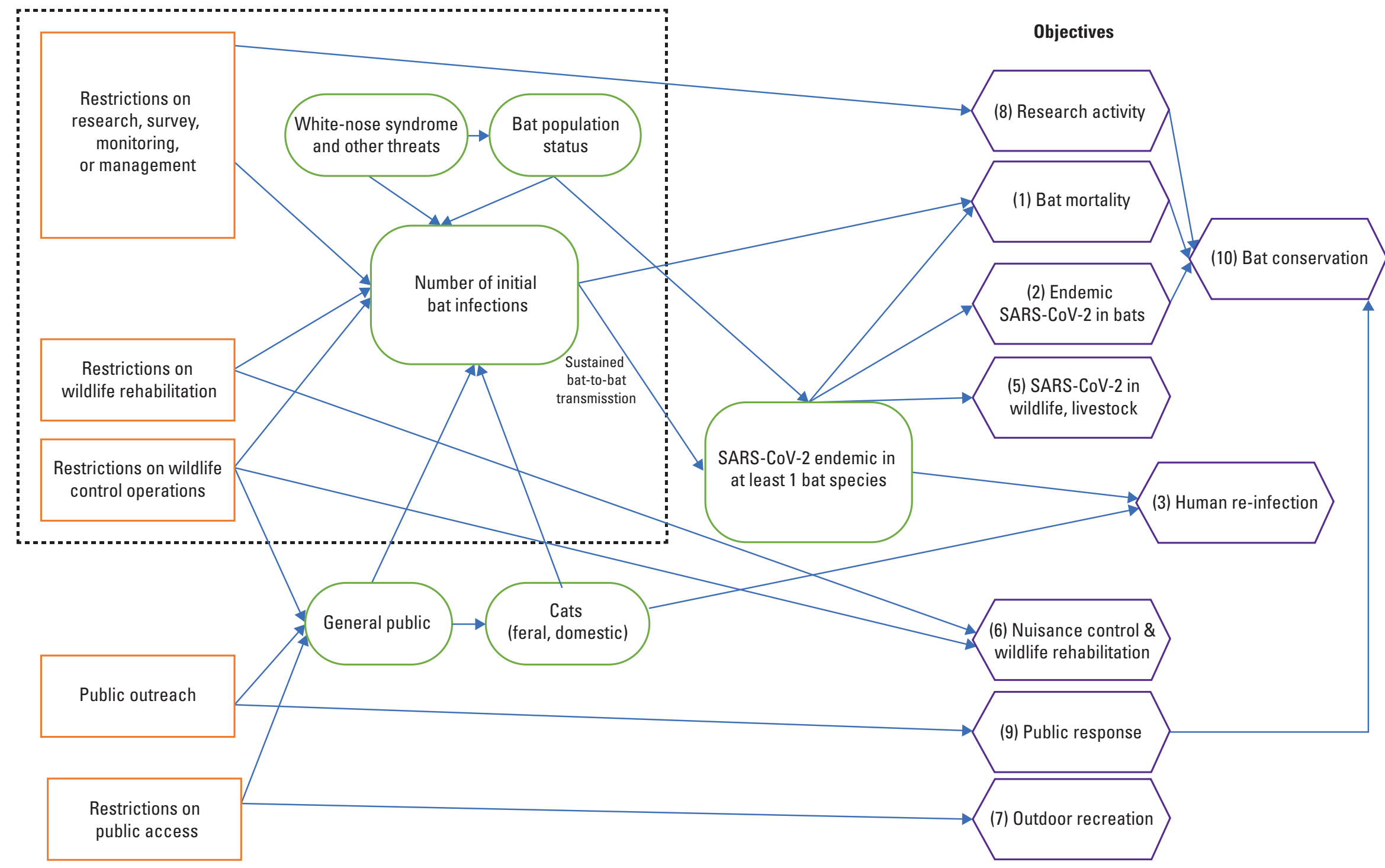

Figure 1. The causal linkages between actions and outcomes motivated by the risk of SARS-CoV-2 entering North American bat populations. The diagram includes potential mitigation actions (orange rectangles), key system states (green ovals), and long-term objectives (blue hexagons). The numbering of the objectives corresponds to the numbering in the "Management Objectives" section of this report. The portion of the diagram within the dashed line emphasizes the initial transmission dynamics analyzed in this assessment. 


\section{Mitigation actions}

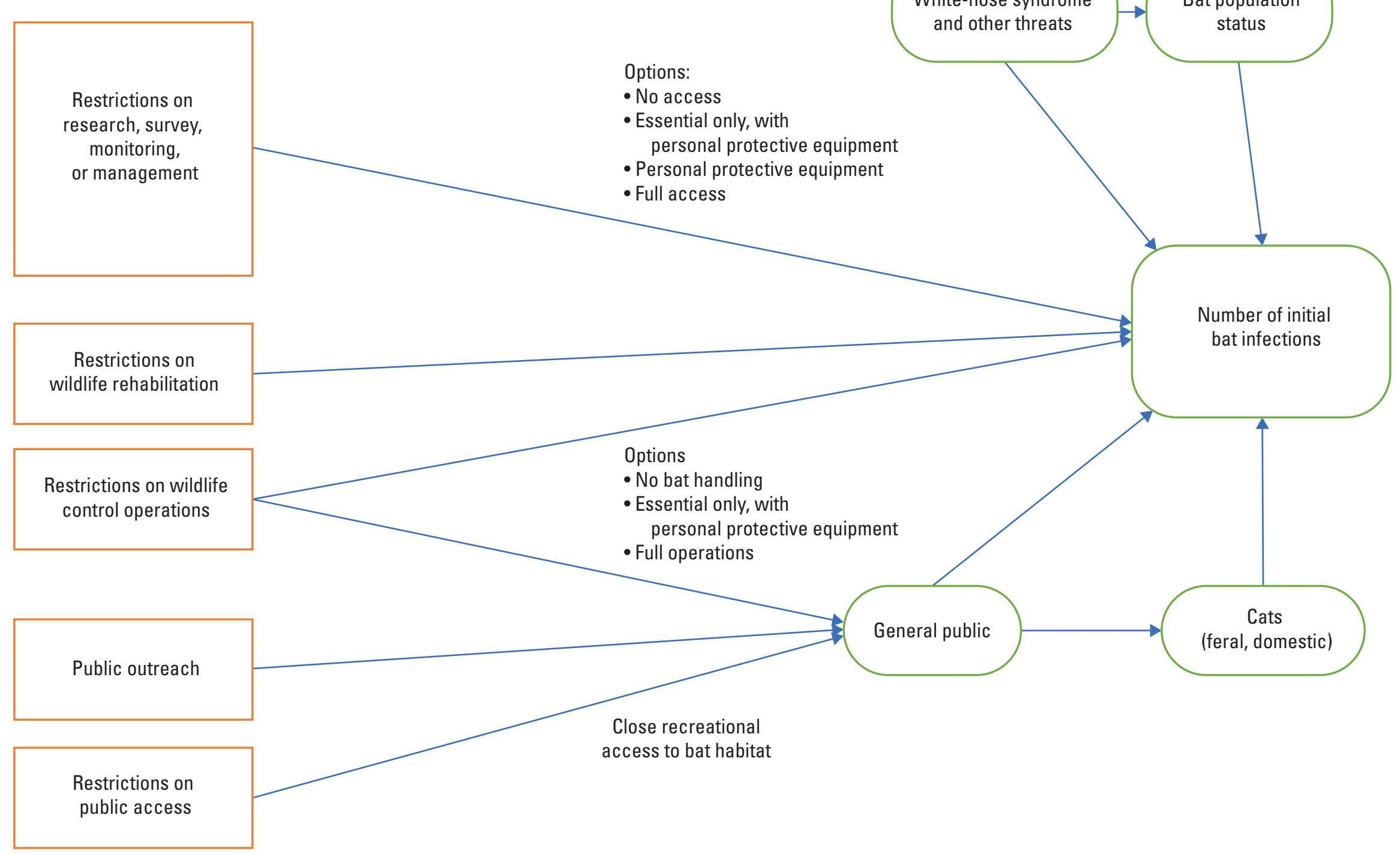

Figure 2. The part of the influence diagram that focuses on the routes of initial transmission of SARS-CoV-2 from humans to bats, with different levels of potential mitigation strategies. 


\section{Focal Questions}

For this initial risk assessment, we wanted to answer the following five questions, recognizing that they are only a small subset of all the questions embedded in figure 1. These initial questions focus on the transmission risk from humans to bats and begin to address the likelihood of sustained transmission in bats. As a starting point, we chose the little brown bat as a surrogate for North American bats because of their widespread distribution (most of the United States north of Arizona, New Mexico, Texas, and Louisiana, and most of Canada south of the Northwest Territories and Nunavut) and frequent contact with humans (Fenton and Barclay, 1980). We chose to focus on the transmission pathways that may be directly affected by guidance from wildlife and research agencies. Finally, we chose to focus our assessment of risk over the near term (that is, the next 6 months), between April and the end of the 2020 bat active season (November, the beginning of hibernation). The five questions below focus on the baseline probability of transmission and the potential effect of mitigation strategies on the transmission probability. In later sections, we use these questions to motivate a mathematical model, then to develop methods, including expert elicitation, to estimate the relevant parameters.

1. Thinking specifically about little brown bats throughout their range in North America, how many individual wild bats would be infected by SARS-CoV-2 directly from humans undertaking research, survey, monitoring, or management (RSM) activities between now (April 2020) and the initiation of hibernation in autumn 2020, in the absence of any new restrictions, regulations, or protocols?

2. Thinking specifically about little brown bats throughout their range in North America, how many individual bats would be infected by SARS-CoV-2 and released into the wild by humans engaged in wildlife rehabilitation (WR) between now (April 2020) and the initiation of hibernation in autumn 2020, in the absence of any new restrictions, regulations, or protocols?

3. Thinking specifically about little brown bats throughout their range in North America, how many individual wild bats would be infected by SARS-CoV-2 directly by humans engaged in wildlife control (WC) between now (April 2020) and the initiation of hibernation in autumn 2020 , in the absence of any new restrictions, regulations, or protocols?

4. (a) How much might the transmission risk owing to research, survey, monitoring, and management (Question 1) be reduced if the fieldwork protocols and guidance included all the following:

- restriction of fieldwork to people without symptoms or contact with someone who had symptoms of COVID-19 in the last 14 days,
- training and oversight in the proper use of PPE,

- proper use of Tyvek or other dedicated clothing,

- proper use of an appropriate N95 respirator, and

- proper use of gloves when handling bats.

(b) Is there any reason to believe this reduction in transmission risk owing to proper use of PPE would be different for wildlife rehabilitators or wildlife control operators compared to scientists or biologists engaged in research, survey, monitoring, and management?

5. What is the basic reproduction number, $R_{0}$, for SARSCoV-2 in little brown bats during the active season? That is, for each infected little brown bat within a naïve population, how many other little brown bats would become infected with the virus?

Questions 1-3 are designed to estimate the probability of infection of bats with SARS-CoV-2 via three pathways of exposure (research and management, wildlife rehabilitation, and wildlife control operations), in the absence of any new guidance (status quo). Question 4 addresses the efficacy of PPE to reduce exposure and subsequent infection. Question 5 provides a rough estimate of the likelihood of sustained bat-to-bat transmission.

This initial focus leaves out many questions of interest. It does not address indirect pathways of transmission (for example, via the general public's contact with bats in their homes or during recreation, or via domestic and feral cats). The initial focus here is on pathways that State and Federal wildlife agencies may be able to directly interrupt. Likewise, this initial focus does not address the effects on the other fundamental outcomes of interest (including mortality of bats, transfer back to humans; fig. 1), but it does address the primary node through which the risk flows (exposure and infection of bats from humans). Because of the urgency to consider interventions available to management agencies in preventing transmission of SARS-CoV-2 from humans to bats, the focus of this assessment is on the pathways that can be affected in the near term.

\section{Model for the Direct Transmission Pathways}

The first three questions listed above address three transmission pathways. Because the pathways are fairly complex, we believed that it would be difficult for experts to directly answer Questions 1-3. Instead, for these questions, we have developed a mathematical model for the component elements of the pathways, and we focused elicitation on those elements.

For the first pathway, we consider the following model, which describes individual components of the transmission process from humans engaged in research, survey, monitoring, or management of bat populations. The model combines the average infection status of humans working with bats, the number of bats encountered (directly through handling and 
indirectly by proximity), the probability of exposing the bats to the virus, and the probability a bat can be infected once it is exposed:

$$
I_{M L}^{R S M}=p_{R S M}^{+}\left(H_{M L}^{R S M} \beta_{H}^{R S M}+E_{M L}^{R S M} \beta_{E}^{R S M}+P_{M L}^{R S M} \beta_{P}^{R S M}\right) \sigma_{M L}
$$

where $I_{M L}^{R S M}$

is the number of little brown bats (ML) directly infected over the course of the 2020 active season by people engaged in research, survey, monitoring, or management (RSM);

$p_{R S M}^{+} \quad$ is the probability that someone conducting such work is actively shedding SARSCoV-2 virus on any given day of the 2020 active season;

$H_{M L}^{R S M} \quad$ is the total number of little brown bats physically handled by any RSM scientist over the course of the 2020 active season; $\beta_{H}^{R S M} \quad$ is the probability that a bat handled by an RSM scientist who was actively shedding virus would be exposed to the virus (an "exposure probability"), in the absence of any new restrictions, regulations, or protocols, taking into account the handling time typical of RSM activities;

$E_{M L}^{R S M} \quad$ is the total number of little brown bats encountered by any RSM scientist within a 6-foot proximity in an enclosed space (such as a cave or attic), without handling, over the course of the 2020 active season;

$\beta_{E}^{R S M} \quad$ is the probability that a bat in an enclosed space within a 6-foot proximity of (but not handled by) a RSM scientist who was actively shedding virus would be exposed to the virus (an "exposure probability"), in the absence of any new restrictions, regulations, or protocols;

$P_{M L}^{R S M} \quad$ is the total number of little brown bats encountered by any RSM scientist within a 6-foot proximity but not in an enclosed space, without handling, over the course of the 2020 active season;

$\beta_{P}^{R S M} \quad$ is the probability that a bat not in an enclosed space but within a 6-foot proximity of (and not handled by) an RSM scientist who was actively shedding virus would be exposed to the virus (an "exposure probability"), in the absence of new restrictions, regulations, or protocols; and

$\sigma_{M L} \quad$ is the probability that a little brown bat exposed to a sufficient viral dose of SARSCoV-2 would actually become infected by the virus (the "infection probability").

Note that we are separating the processes of exposure and infection. By exposure probability, we mean the likelihood that a particular interaction between an average bat and a person who is actively shedding SARS-CoV-2 virus will result in exposure of the bat to a sufficient viral dose to cause infection. By infection probability, we mean the probability that the virus replicates in the host (bat) tissue, conditional on that bat having been exposed to a sufficient viral dose. That is, the exposure process is about whether enough virus was transferred to make an infection possible; it is a property of the interaction between the biologist and that bat. The infection process is about the molecular, cellular, immunological, and physiological conditions that allow viral replication in the bat; it is a property of the interaction of the bat and the pathogen.

The parameters $H_{M L}^{R S M}, E_{M L}^{R S M}$, and $P_{M L}^{R S M}$ were estimated by surveying State, Federal, and research agencies engaged in bat research (see "Methods" section). The parameter $p_{R S M}^{+}$was estimated from human epidemiological models of COVID-19, accounting for the expected cumulative incidence over the 2020 active season and the average length of time that an infected person is shedding virus (see "Methods" section). At this time, in the absence of empirical data, the three exposure probabilities, $\beta_{H}^{R S M}, \beta_{E}^{R S M}$, and $\beta_{P}^{R S M}$, and the infection probability, $\sigma_{M L}$, had to be estimated through expert elicitation.

We describe the second pathway of transmission, through the activities of wildlife rehabilitators, in a similar manner:

$$
I_{M L}^{W R}=p_{W R}^{+}\left(H_{M L}^{W R} \beta_{H}^{W R}+P_{M L}^{W R} \beta_{P}^{W R}\right) \sigma_{M L}
$$

where the subscripts and superscripts now refer to people engaged in wildlife rehabilitation (WR), but the parameters are analogous to equation 1 . Note that in this equation, there are only two "distances" of interaction: handling $(H)$ and proximity $(P$, here meaning within a 6-foot proximity, whether enclosed or not); we expect that, in most cases, the interactions that wildlife rehabilitators have with bats involve handling, typically for more extensive periods of time than in other pathways.

The parameters $H_{M L}^{W R}$ and $P_{M L}^{W R}$ were estimated by surveying State agencies that permit or otherwise authorize wildlife rehabilitation organizations (see "Methods" section). At this time, the two exposure rates, $\beta_{H}^{W R}$ and $\beta_{P}^{W R}$, were estimated through expert elicitation because we lack empirical estimates.

We assumed that the parameter $p_{W R}^{+}$did not differ from $p_{R S M}^{+}$. Likewise, we assumed the infection rate conditional on exposure, $\sigma_{M L}$, is the same in equations 1 and 2 , as well as equation 3 below. That is, we assumed the infection rate is a function of the bat species but does not differ on the basis of the route of transmission because the effects of the route of transmission are captured in the exposure rates.

We describe the third pathway of transmission, through the activities of wildlife control operators, in a similar manner:

$$
I_{M L}^{W C}=p_{W C}^{+}\left(H_{M L}^{W C} \beta_{H}^{W C}+P_{M L}^{W C} \beta_{P}^{W C}\right) \sigma_{M L}
$$

where the subscripts and superscripts now refer to people engaged in wildlife control operations (WC), but the parameters are analogous to those in equation 2. 
The parameters $H_{M L}^{W C}$ and $P_{M L}^{W C}$ were estimated by surveying State agencies that permit or otherwise authorize wildlife rehabilitation organizations (see "Methods" section). The two exposure rates, $\beta_{H}^{W C}$ and $\beta_{P}^{W C}$, were estimated through expert elicitation. As in equation 2, we assume that $p_{W C}^{+}$is identical to $p_{R S M}^{+}$and that $\sigma_{M L}$ is the same across transmission pathways.

Equations 1, 2, and 3 specifically focus on the transmission rates under status quo conditions, by which we mean the ways wildlife biologists and other professionals in the United States most likely would have interacted with bats prior to the arrival of SARS-CoV-2 in North America. In particular, past concern about transfer of biological agents was primarily focused on rabies virus, histoplasmosis, and the fungus $P$. destructans. Typical protocols for $P$. destructans involve decontamination of clothing and footwear between sites and wearing nitrile gloves (with disposal or decontamination between bats), but use of face masks or respirators was not implemented or continued once it was determined humans were unlikely to be harmed by the fungus. Face masks are employed only in specific situations, primarily when working amidst large amounts of bat guano. We were also interested in assessing whether updating guidance and protocols for work with bats (including use of PPE) can reduce the transmission rates. To do this, we assume that the effect of such guidance is on the exposure parameters (the $\beta$ parameters). For this initial risk assessment, we focus on guidance that allows all work with bats to continue but includes all of the mitigations described in Focal Question 4. We estimated the change in the $\beta$ parameters as a result of use of enhanced PPE through expert elicitation.

\section{Methods}

\section{Expert Elicitation}

We used expert elicitation methods to estimate 12 parameters for which data are not yet available (the seven exposure rates in equations 1,2 , and 3 ; the infection rate in equations 1,2 , and 3 ; three multipliers of exposure rate that reflect the effect of enhanced PPE; and the basic reproduction number, $R_{0}$, for SARS-CoV-2 in little brown bats; see appendix 1). To reduce the effects of expert bias and overconfidence on the results, we conducted an elicitation using a modified Delphi process that includes two rounds of elicitation with feedback and discussion in between. Six steps were followed: (1) choosing experts, (2) training experts, (3) conducting a first round of elicitation, (4) reviewing and discussing the first round with the experts, (5) allowing experts to adjust their assessments in a second round of elicitation, and (6) aggregating the estimates across experts (Hanea and others, 2017). The modified Delphi approach requires experts to share and discuss the logic behind their opinions. In addition, to capture within-expert uncertainty, the four-point elicitation procedure was used (Speirs-Bridge and others, 2010), which consists of asking experts for their lowest, highest, and best estimates, and their confidence the true value lies within the reported interval. By using the scientific judgments from multiple experts, we included uncertainty in scientific understanding in the predictions of the effect of mitigation actions on the desired outcomes.

Through literature review and the professional contacts of two of the authors of this report (P.M. Cryan and K.J. Olival), a list was compiled of 43 experts whose expertise spans bat ecology, virology (especially of coronaviruses), epidemiology, and wildlife disease, with an emphasis on experts who had a demonstrated background in one or more of those fields. Seventeen experts from this list were invited to participate in the elicitation. The invited participants were selected to produce a group with diverse and complementary scientific backgrounds, from several countries, with a balanced gender representation. The invited participants included scientists from academic institutions, government agencies, and non-governmental organizations (see Acknowledgments).

\section{Training}

Formal expert elicitation is a new process for many, so the first step was to familiarize experts with the approach. This training step increases the quality of expert judgment for unknown qualities (Cooke, 1991). Before starting the elicitation concerning the questions of interest, we provided the expert panel a chance to practice the elicitation methods. Questions were provided for which the answers were known (that is, answers were identified values from the literature, but they were unlikely to be known precisely by experts). The questions were used to ensure that the instructions were understood by the experts and to allow the experts a chance to self-calibrate their estimates of uncertainty. We asked three questions (Appendix 1) - one for which we assumed the uncertainty had a normal distribution (forearm length of a little brown bat), one for which we assumed a log-normal distribution of uncertainty ( $R_{0}$ of WNS), and one for which we assumed a logit-normal distribution (breeding probability) - which represented the kinds of parameters we would ask in the real elicitation. Experts were provided with a custom spreadsheet to record their answers, which plotted the probability distribution resulting from their responses. We did not use responses to the training questions to rank expert quality or weight experts when summarizing responses for the model.

The results of the training questions were summarized and sent to the experts prior to the first elicitation round. The exercise revealed some potential for linguistic uncertainty in two of the questions ( $R_{0}$ and breeding probability), which we discussed in feedback to the experts prior to the first elicitation round. 


\section{Round 1}

The experts were provided with a document that described the background of the study, a summary (a draft synthesis manuscript, including literature references) of the current state of knowledge on SARS-CoV-2 and bats, a detailed description of the quantities that were being elicited (see Appendix 1), instructions for completing the elicitation, and a spreadsheet that plotted the distribution that represented their uncertainty from their responses to the four-point elicitation. The experts were asked to work independently and return their responses within about 72 hours.

\section{Group Discussion}

The results from the first round of elicitation were compiled; for each question, the anonymous responses for each of the experts were shown graphically (for example, appendix fig. 2.1A), the fitted distribution for each expert was plotted (for example, fig. 2.1B), and the average distribution across experts was plotted (for example, fig. 2.1C). Two 2-hour video conference calls were held with the experts to discuss the first round of results, focusing on several topics: clarifying the interpretation of the questions, sharing insights of individual experts, discussing notable differences in how the experts answered the questions, and reinforcing the instructions for the four-point elicitation method. After the calls, the results of the first round and a brief written summary of the discussion (especially, to clarify the questions) were provided to the experts.

\section{Round 2}

The experts were asked to reconsider their responses to the questions, taking into account the group discussion, and independently provide a revised set of answers within 24 hours.

\section{Aggregation of Experts}

Separately for each parameter and expert, we fitted a probability distribution to the responses to the four-point elicitation, assuming the best estimate corresponded to the median and the confidence limits represented symmetric quantiles. We assumed that questions $1-8$ were best represented with logit-normal distributions because the quantities elicited were proportions bounded by 0 and 1 , which is the support for the logit-normal distribution. We assumed that questions 9-11 and 13 were best represented with lognormal distributions because the quantities elicited were bounded by 0 on the lower end and unbounded on the upper end, like the lognormal distribution. We fitted the distributions by regressing the inverse cumulative distribution function of the quantiles against the corresponding values.
The individual probability density functions (PDF) were aggregated, with equal weight, across experts. We then found the parameters of a logit-normal or lognormal distribution that best fit the average PDF, as measured by the Kullback-Leibler distance (Kullback and Leibler, 1951). The fitted, aggregated distribution provided an estimate, with uncertainty, for each parameter.

\section{Other Parameters}

\section{Bat Handling Data}

Seven State and four Federal wildlife agencies were queried for bat research or permit records to estimate the number of bats handled over the course of a field season for the three transmission pathways. The State and Federal agencies queried were a non-random sample, composed primarily of agencies that were on the guidance committee (see Acknowledgments).

\section{Probability an Individual Human is Shedding SARS-CoV-2}

To estimate the probability that a bat worker would be shedding SARS-CoV-2 at the time of an encounter with a bat ( $p_{R S M}^{+}$in equation 1$)$, three components were considered: cumulative incidence, which is the probability of the worker becoming infected with SARS-CoV-2 in the United States from April 15 through November 15, $2020\left(C^{+}\right)$; the length of time an individual with COVID-19 is shedding virus $(s)$; and the length of the period of time in question $(t, 214$ days, April 15-November 15, 2020). Then, the desired probability is given by

$$
p_{R S M}^{+}=C^{+} * \frac{s}{t} .
$$

Regarding the cumulative incidence $\left(C^{+}\right)$, although a number of models can forecast SARS-CoV-2 infection in humans, most report only the peak number of hospital admissions and intensive care unit beds or provide forecasts for only the next approximately 4 months. We could not find any models that specifically forecast the cumulative incidence between April 15 and November 15, 2020, but there are a few models that forecast the incidence through the course of the epidemic. If we assume that the bulk of the epidemic in the United States will occur by November 15, those forecasts are helpful benchmarks. The forecast of Moghadas and others (2020) was used, which estimates the cumulative incidence for the COVID-19 epidemic in the United States under two values of $R_{0}$ for SARS-CoV-2 in humans $(2.0,2.5)$, resulting in 177 million and 233 million people infected through the end of the epidemic (representing 53.8 and 70.5 percent, respectively, of the total U.S. population infected, assuming a U.S. population 
size of 329.1 million, https://www.census.gov/popclock/; accessed April 20,2020). These estimates are similar to the forecast of Ferguson and others (2020) for 81 percent of the U.S. population to be infected, if $R_{0}$ is 2.4 . We treated the two estimates from Moghades and others (2020) as the median and 90th quantile of a logit-normal distribution. For the 10th quantile for the cumulative incidence of COVID-19 disease in the United States by November 15, 2020, we divided the number of reported cases on April 20 (788,172; https://www.cdc.gov/ coronavirus/2019-ncov/cases-updates/cases-in-us.html) by a reporting rate of 0.066 for the United States (Bommer and Vollmer, 2020) and multiplied by 3 (assuming mid-April was roughly the peak infection rate in the United States, and the decline will involve three times as many cases as the increase); the result is a 10.9-percent cumulative infection rate through November 15. We fitted these three quantiles (10th, 50th, and 90th) to a logit-normal distribution.

We estimated shedding duration $(s)$ using results from Zhou and others (2020a) who found a median duration of SARS-CoV-2 shedding for hospitalized patients to be 31.0 days from illness onset (interquartile range [IQR]: 24.0-40.0, minimum: 18, maximum: 48). Other studies (Wölfel and others, 2020; Zou and others, 2020) report shorter maximum durations, but that may be related to the lengths of observation studies. A linear model fitted to figure $1 \mathrm{~b}$ of Wölfel and others (2020) estimates a maximum of 25 days of shedding. Zou and others (2020) report detection of viral DNA up to the end of the study period ( 21 days). To capture uncertainty in the duration of viral shedding, we fitted a normal distribution to the values $(18,31,48)$, associating those values with three quantiles $(0.025,0.50,0.975)$.

\section{Simulation Model}

To integrate the estimates of the parameters in equations 1,2 , and 3 , and to propagate the uncertainty in those estimates through to the results, we built a Monte Carlo simulation model for those equations. We sampled each parameter from the probability distribution that represented its estimate. In most cases, the parameters were sampled independently, but for the 3 RSM exposure probabilities (the $\beta$ parameters in equation 1), the 2 WR exposure probabilities (the $\beta$ parameters in equation 2), the $2 \mathrm{WC}$ exposure probabilities (the $\beta$ parameters in equation 3), and the three PPE multipliers, we assumed that the parameters in those sets had a correlation of 0.50 to reflect the assumption that, for example, if the true exposure probability for RSM handling was on the high end of its uncertainty distribution, the true exposure probability for RSM proximity is likely to be at the high end of its uncertainty distribution. For each of 1,000 replicates in the simulation, equations 1 through 3 were used to calculate the number of infected bats from the sampled parameter values.

\section{Results}

\section{Encounters with Bats}

Federal and State wildlife agencies do not have a common system for documenting and recording encounters with bats by scientists, rehabilitators, and wildlife control operators, so the data on encounters were difficult to compare across agencies (table 1). Bat research, survey, monitoring, and management activities span all three types of encounter, with a mixture that depends on how the agency in question permits such activities, as well as the needs of the specific setting. Across agencies that were able to report encounters for all three modes, 45.8 percent involved handling, 11.5 percent involved proximity in an enclosed space, and 42.7 percent involved proximity in an unenclosed space. Wildlife rehabilitation invariably involves handling, not simply proximity to bats. Wildlife control operations have a mixture of activities that involve handling or proximity. Across the States that reported totals for two modes of encounter, 22.9 percent of the encounters by wildlife control operators involved handling, and 77.1 percent involved proximity without handling.

After the data in table 1 were collected from the wildlife agencies, we realized several unanticipated challenges: (1) the data are not collected in a common way across agencies, making comparisons difficult; (2) there may be duplicate counts because the same activity may be recorded by multiple agencies (for example, a research project conducted on USFS land in Virginia may be recorded both by USFS and by the Virginia Department of Game and Inland Fisheries); (3) it was not possible, in the available time frame, to gather comprehensive data across the United States; and (4) in many cases, the data on encounters are recorded for all bats, not by species. In light of these challenges, we were not able to estimate the $H, E$, and $P$ parameters in equations 1,2 , and 3 . Instead, we used the data in table 1 to estimate relative values for $H, E$, and $P$. Thus, in the results that follow, the number of potential infections is not expressed on an absolute scale, but on a relative scale, reflecting the probability of infection.

\section{Expert Judgment}

Of the 17 experts invited to participate in the elicitation process, 13 participated in either Round 1 or Round 2; in the final analysis, we included only the 12 experts who had participated in the group discussions and who submitted Round 2 estimates afterwards. The four-point responses, the individual fitted distributions, the mean aggregate distribution, and the fitted aggregate distribution are shown for each question in Appendix 2. 
Table 1. Number of bats encountered during the 2019 active bat season.

[The entries show the number of bats of any species encountered during the 2019 active bat season, April 15-November 15, 2019, as reported through direct requests to the agencies listed in the table. Blank entries indicate only that the quantity was not recorded or estimated by the agency, whereas an entry of 0 indicates that the agency recorded no encounters in that category. Gray cells indicate no encounters were expected because the agencies in question do not oversee rehabilitation or wildlife control operations. CO CPW, Colorado Parks and Wildlife; CT DEEP, Connecticut Department of Energy and Environmental Protection; KY DFWR, Kentucky Department of Fish and Wildlife Resources; NYSDEC, New York State Department of Environmental Conservation; OR DFW, Oregon Department of Fish and Wildlife; VA DGIF, Virginia Department of Game and Inland Fisheries; WI DNR, Wisconsin Department of Natural Resources; FS, Forest Service; NPS, National Park Service; USGS, U.S. Geological Survey; WNS, white-nose syndrome surveillance program; k, thousand]

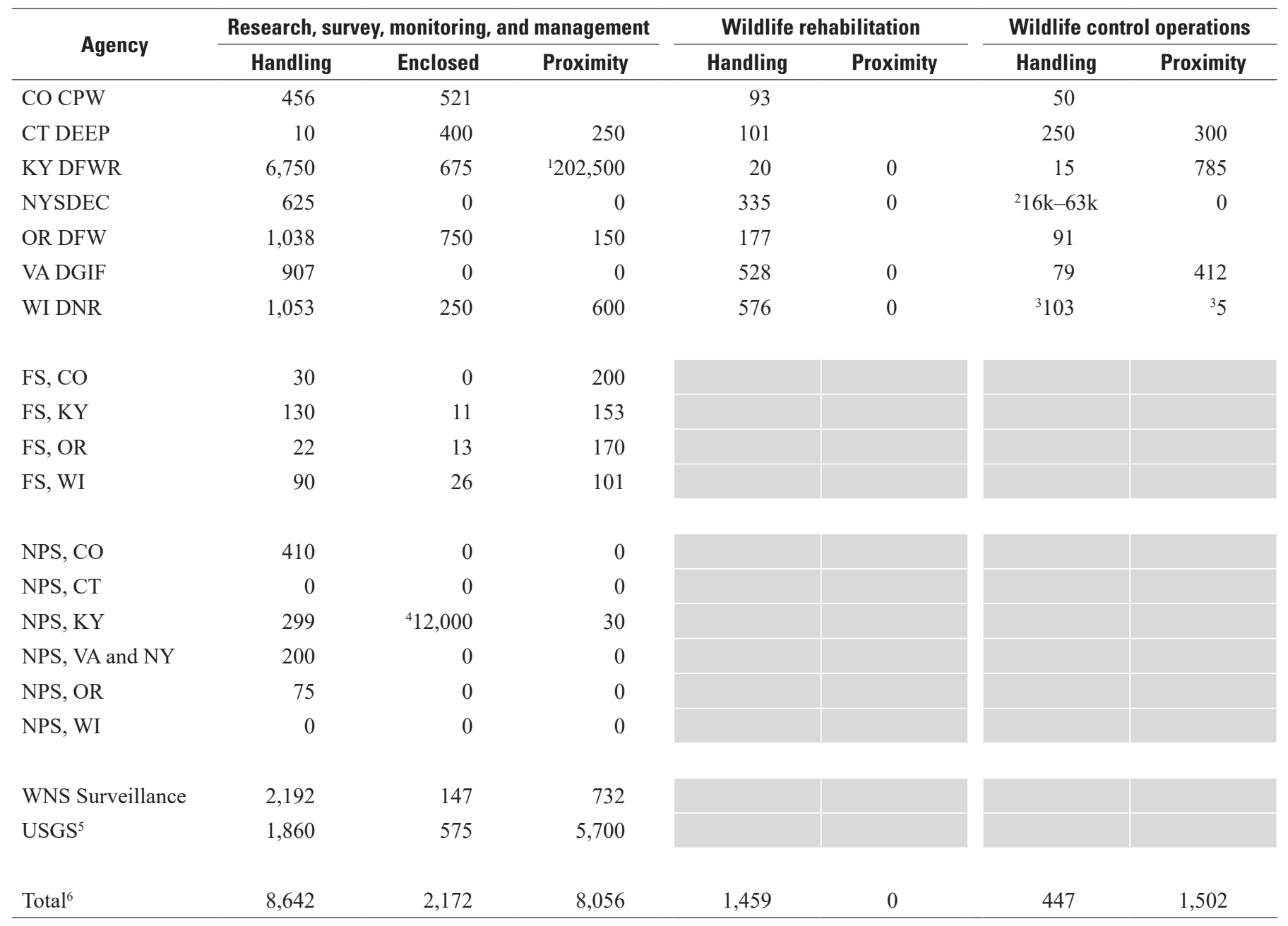

${ }^{1}$ The large number of bats in the proximity of scientists arises primarily from emergence counts from roost sites.

${ }^{2}$ This estimate includes bats handled by homeowners as well as wildlife control operators.

${ }^{3}$ Includes only wildlife control operators with permits to handle State threatened and endangered species (possibly only 25-30 percent of operators).

${ }^{4}$ This includes bats within 6 feet of tour guides at Mammoth Cave National Park over the duration of the summer.

${ }^{5}$ This includes all USGS scientists within the Ecosystems Mission Area who work on bats.

${ }^{6}$ For all columns within each category (Research, survey, monitoring, and management; Wildlife rehabilitation; Wildlife control), the total includes those agencies that reported entries for each mode of handling in the category, without a noted exception as to how the encounters were interpreted. That is, to be included in the totals for a category, an agency needed to report entries for each of the handling modes, and there needed to be no exceptions noted on any of the entries. If these conditions were not met, then none of the entries for that agency were included in the totals for that category. 
For RSM workers, the detailed responses for the three exposure probabilities (Q1, Q2, and Q3) are shown in Appendix figures 2.1, 2.2, and 2.3, and the fitted aggregate distributions are summarized in figure 3 . Using the fitted aggregate distribution, we estimated that a median 49.7 bats per 100 handled by actively shedding SARS-Co- $\mathrm{V}-2$ virus $(\mathrm{CoV}+)$ RSM workers would be exposed to a sufficient dose of virus for infection (80-percent interval, 15.3-84.3); this is close to a uniform distribution between 0 and 100 , indicating the experts had considerable uncertainty about the degree of this exposure. For Q2, the aggregated expert judgment is that a median 19.4 bats per 100 encountered within an enclosed space by CoV+ RSM workers would be exposed to a sufficient dose of virus to possibly lead to infection (80-percent interval, 2.2-72.4). For Q3, the aggregated expert judgment is that a median 6.4 bats per 100 encountered within 6 feet in an unenclosed space by $\mathrm{CoV}+\mathrm{RSM}$ workers would be exposed to a sufficient infectious dose of virus (80-percent interval, 0.6-43.8). Thus, although the experts expressed considerable uncertainty in the range of these exposure probabilities, the pattern showed a consistent decrease as the nature of the encounter became less proximal (fig. 3). Similar types of results were estimated for the exposure rates through the wildlife rehabilitation (figs. 2.4 and 2.5) and wildlife control (figs. 2.6 and 2.7) transmission pathways.

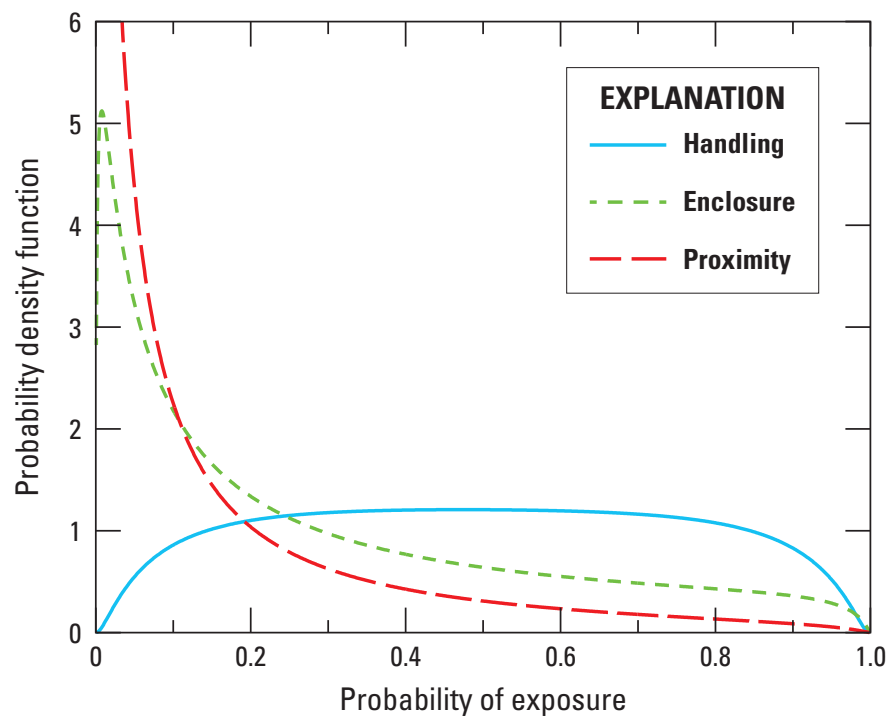

Figure 3. Aggregate probability distributions for the probability of exposure of bats from a SARS-CoV-2-positive research, survey, monitoring, and management worker, referenced in expert judgment questions 01,02 , and 03 .
Conditional on exposure to a sufficient dose of SARS$\mathrm{CoV}-2$, the experts estimated that the median probability a little brown bat would develop an infection was 0.44 (80-percent interval, 0.08-0.88; fig. 2.8). During discussion, the experts noted their particular uncertainty about this parameter (represented by the nearly uniform aggregate distribution) and noted that this parameter is a critically important nexus in the causal diagram (fig. 1).

The experts expected that proper use of PPE (that is, an appropriate N95 respirator and other protective gear) would be effective at substantially reducing the exposure probability. The detailed responses of the experts are shown in figures 2.9, 2.10, and 2.11 (Appendix 2) as proportional multipliers on exposure probability. The fitted aggregate distribution, expressed as the percent reduction in exposure (1 minus the quantity elicited), did not differ substantially among the three modes of handling (fig. 4), ranging from a mean of 94 to 96 percent effective. When asked an open-ended question about whether the effectiveness of PPE would differ among scientists (RSM), rehabilitators (WR), and wildlife control operators (WC), the experts were inconclusive; about half thought there would not be tangible differences in the effectiveness of PPE, and the other half thought that differences in compliance among the three groups could possibly affect the amount by which exposure was reduced.

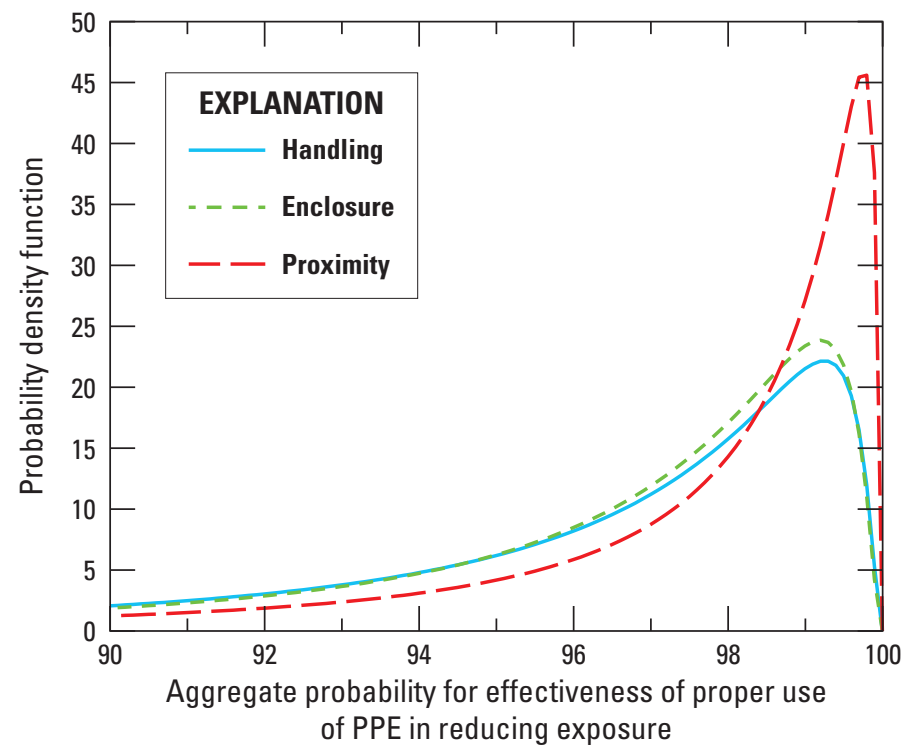

Figure 4. Aggregate probability distributions for the effectiveness of proper use of personal protective equipment in reducing exposure of bats to SARS-CoV-2 by a research, survey, monitoring, and management worker, referenced in expert judgment questions 09,010 , and 011 , expressed as the percent reduction in exposure. 


\section{Probability a Worker is Shedding Virus}

The probability of a worker shedding SARS-CoV-2 virus during an encounter with bats over the course of summer 2020 (April 15-November 15), absent a change in field work practices, was estimated to be 0.057 (that is, 5.7 percent, median; 80-percent interval, 0.022-0.112; fig. 5). This is based on a cumulative incidence of 0.39 (median; 80-percent interval, $0.17-0.71$ ) and a shedding duration of 33 days (median; 80-percent interval, 23-42) within a 214-day field season.

\section{Probability of Bats Being Infected}

Combining the probabilities of exposure and infection, for multiple modes of encounter under status quo conditions, we estimate that a $\mathrm{CoV}+\mathrm{RSM}$ scientist would infect 107 (median) per 1,000 bats (80-percent interval, 16-348); a CoV+ WR worker would infect 252 (median; 80-percent interval, 41-654) per 1,000; and a $\mathrm{CoV}+\mathrm{WC}$ worker would infect 50 (median; 80-percent interval, 5-268) per 1,000 (fig. 6).

Combining the probabilities of exposure and infection (fig. 6) with the probability that a worker is $\mathrm{CoV}+$ (fig. 5),

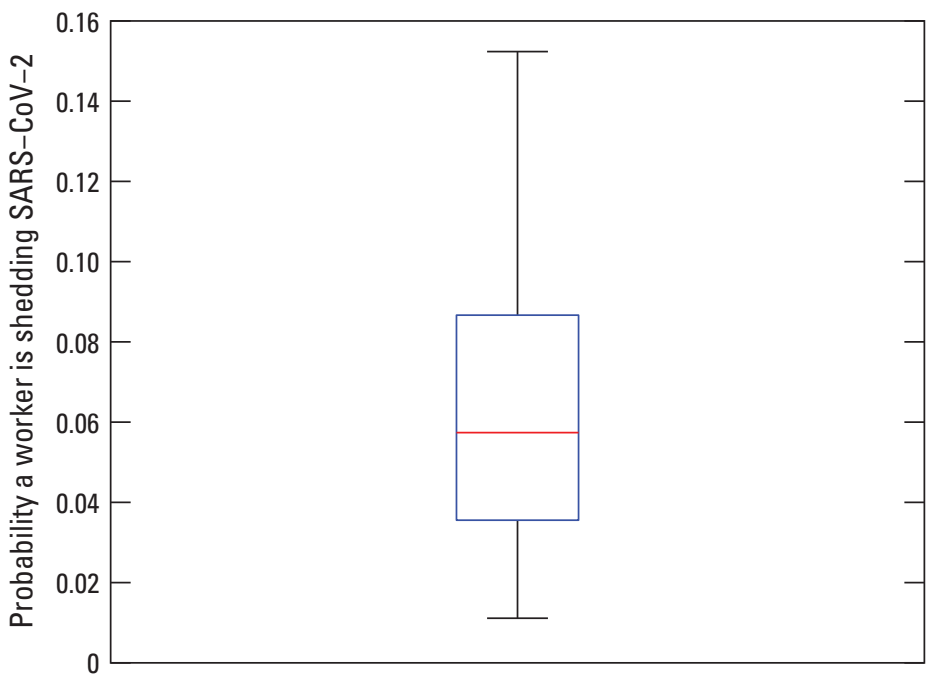

EXPLANATION

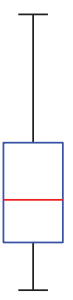
99th percentile

75th percentile

Median Interquartile 25th percentile range

Figure 5. Probability that a worker is shedding SARS-CoV-2 during an encounter with a bat.

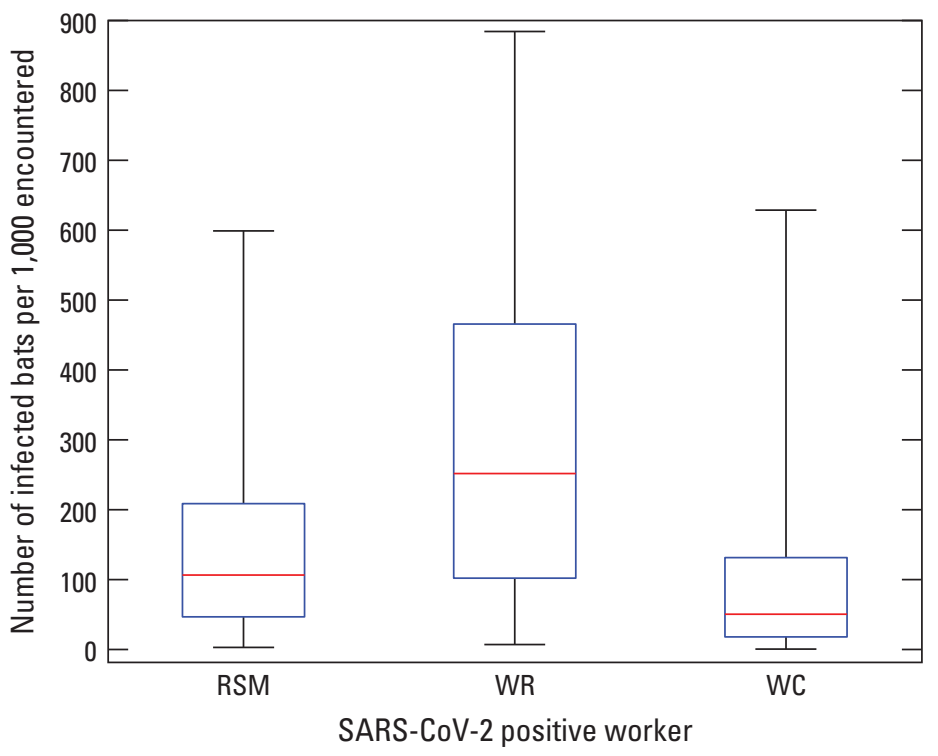

EXPLANATION

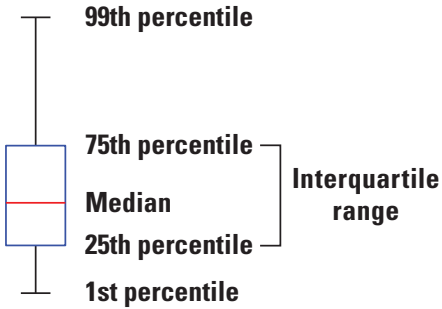

Figure 6. Number of bats per 1,000 exposed to and infected by virus from a SARS-CoV-2-positive worker, by transmission pathway, under status quo working conditions. (RSM, research, survey, monitoring, and management activities; WR, wildlife rehabilitation; WC, wildlife control operations.) These results were obtained with the assumed ratio of encounter modes (handling, enclosure, and proximity) estimated from the totals in table 1. 
we estimate that an average RSM worker would infect 6.0 (median) per 1,000 bats (80-percent interval, 0.8-22.2); an average WR worker would infect 12.7 (median; 80-percent interval, 1.8-42.8) per 1,000; and an average WC worker would infect 2.7 (median; 80-percent interval, 0.3-16.6) per 1,000 (fig. 7), under status quo conditions. But if PPE protocols and training are implemented, the respective probabilities of infection drop to $0.2,0.4$, and 0.1 bats per 1,000 , respectively (median values, with narrow uncertainty ranges; fig. 7).

Another way to understand the results in figure 7 is to consider the probability that the number of infections exceeds some threshold value. For example, the probabilities that at least 5 per 1,000 bats are infected is 55 percent for RSM (1.3 percent with PPE), 75 percent for WR (4.6 percent with PPE), and 35 percent for WR ( 0.6 percent with PPE). We use the exceedance threshold 5 per 1,000 to demonstrate this approach; the relevant threshold value reflects the decision maker's risk tolerance for introduction of infected bats to the wild population.

The effect of the mode of encounter on the infection rate in the RSM transmission pathway is notable (fig. 8). The combined probability that at least 5 bats per 1,000 are exposed to and infected by SARS-CoV-2 from an average RSM worker is 66 percent if handling is involved (3.1 percent with PPE), 40 percent if the bats are encountered in an enclosed space without handling (1.3 percent with PPE), and 24 percent if the bats are encountered in an unenclosed space $(0.4$ percent with PPE).

\section{Probability of Transmission in Bat Populations}

The experts expressed considerable uncertainty about the basic reproduction number $\left(R_{0}\right)$ for SARS-CoV-2 in little brown bats (fig. 9; expert panel responses in fig. 2.12). The bulk of the fitted aggregate distribution was less than 1.0, with a median of 0.45 and an 80 -percent interval of $(0.05,4.38)$, but the experts could not rule out the possibility that SARSCoV-2 could be effectively transmitted in a little brown bat population. For the fitted aggregate distribution, the probability that the basic reproduction number $\left(R_{0}\right)$ is greater than 1 is 32.6 percent.

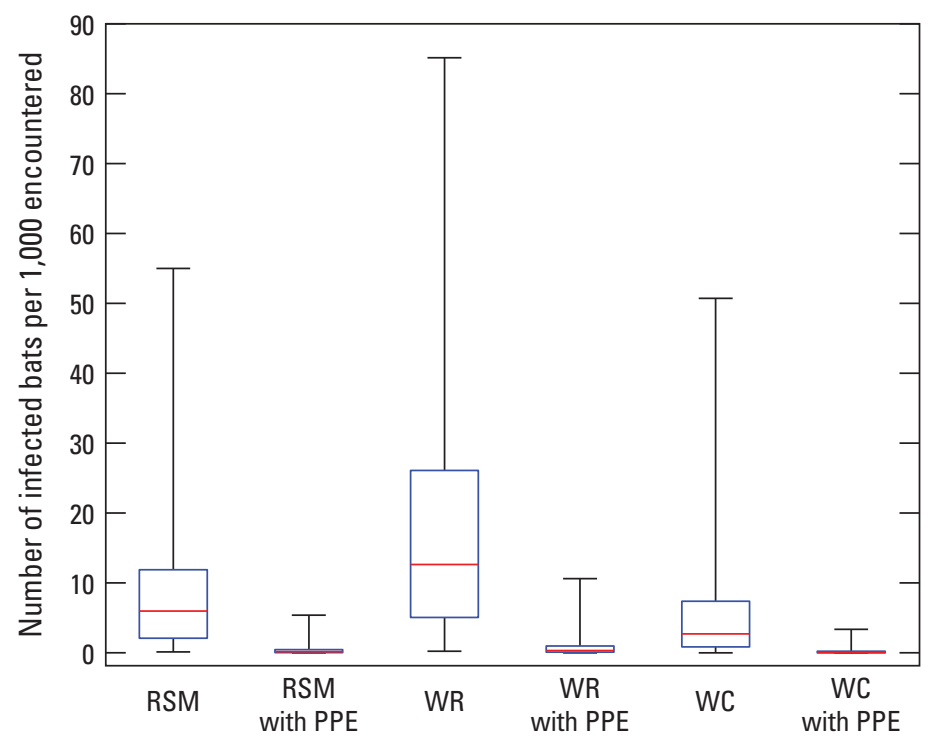

EXPLANATION

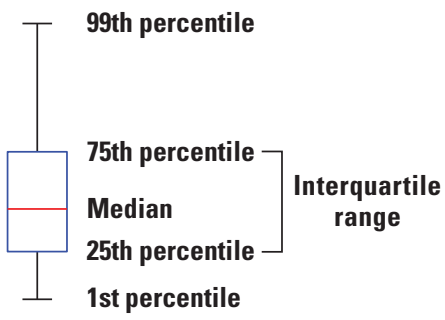

Figure 7. Number of bats per 1,000 exposed to and infected by SARS-CoV-2 from an average worker, by transmission pathway, without and with the proper use of personal protective equipment. (RSM, research, survey, monitoring, and management activities; WR, wildlife rehabilitation; WC, wildlife control operations; PPE, personal protective equipment) 


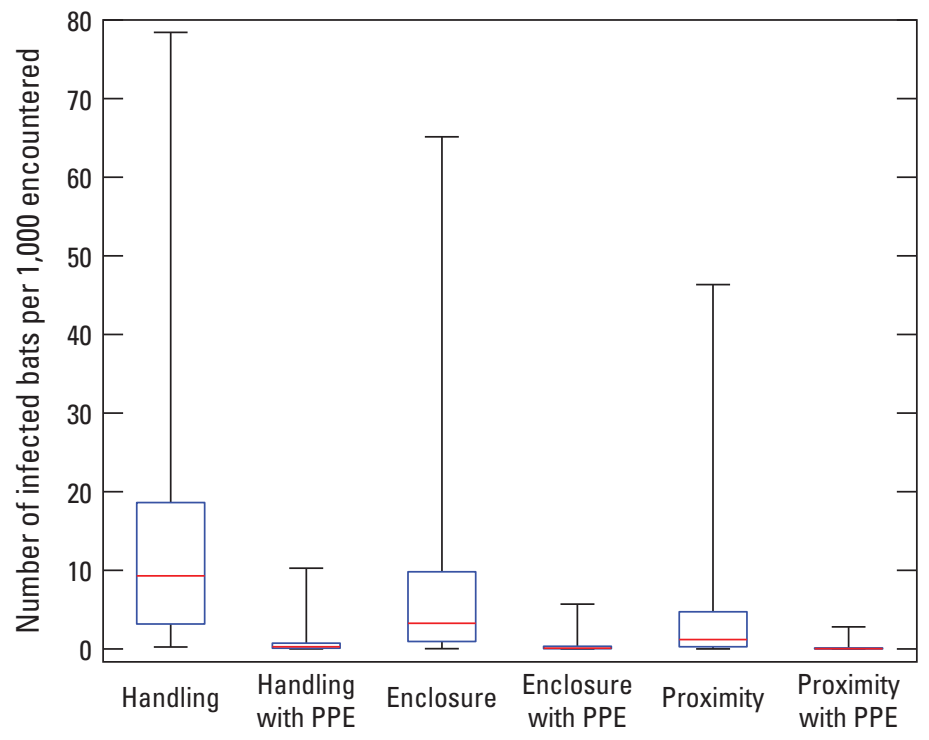

\section{EXPLANATION}

- 99th percentile

\begin{tabular}{|l|l}
\hline & $\begin{array}{l}\text { 75th percentile } \\
\text { Median } \\
25 \text { th percentile }\end{array}$ \\
\hline & $\begin{array}{l}\text { Interquartile } \\
\text { range }\end{array}$ \\
\hline & 1st percentile
\end{tabular}

Figure 8. Number of bats per 1,000 exposed to and infected by SARS-CoV-2 from an average scientist in the RSM pathway, by encounter type, without and with proper use of personal protective equipment. (RSM, research, survey, monitoring, and management activities; PPE, personal protective equipment)

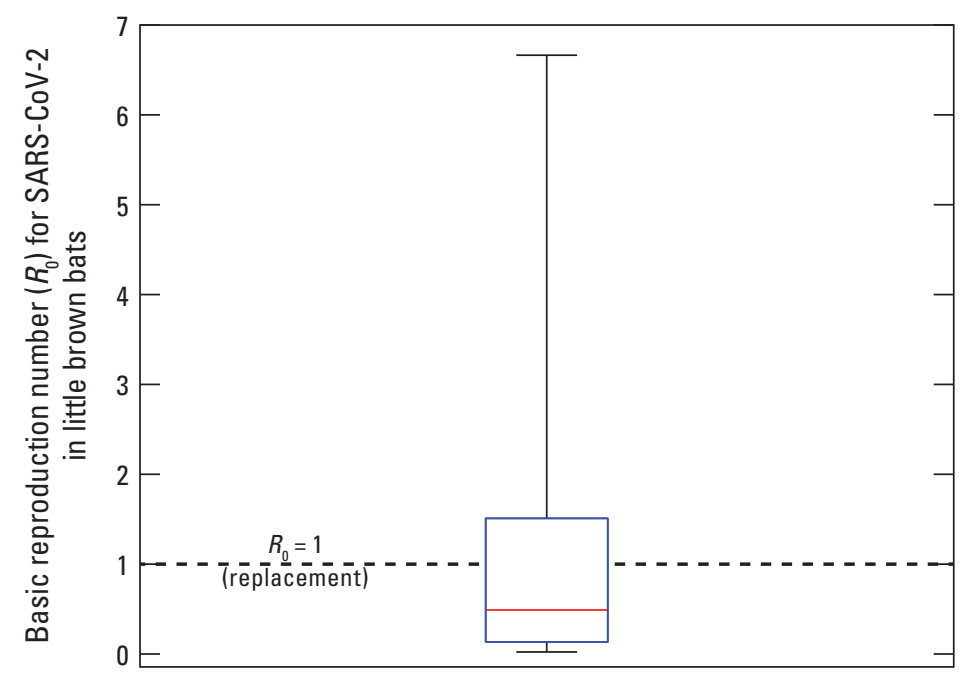

EXPLANATION

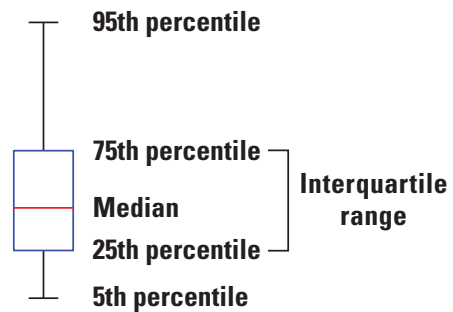

Figure 9. Basic reproduction number, $R_{0^{\prime}}$ for SARS-CoV-2 in little brown bats, as estimated by an expert panel. 


\section{Discussion}

This risk assessment was a response to the concern that the novel $\beta$-coronavirus, SARS-CoV-2, could be transmitted to naïve North American bat populations through human contact, and once infected, bats could experience mortality and become a reservoir for the virus with the potential to re-infect people in the future. The goal of this assessment is to provide timely scientific information to guide State and Federal wildlife management agency response to this potential risk. The rapid emergence of SARS-CoV-2 in human populations created significant uncertainty in forecasting the risk of transmission to native bat populations. Along with decision makers from State and Federal agencies, we framed the management decision using principles of decision analysis, identified important objectives, and articulated potential mitigation actions. By framing the decision explicitly, we were able to provide information specifically useful for risk mitigation.

The World Organisation for Animal Health (OIE) Aquatic Animal Health Code defines a risk analysis as being composed of four parts: hazard identification, risk assessment, risk management, and risk communication (World Organisation for Animal Health, 2019, Chapter 2.1). The first step is the identification of a pathogen which may pose a hazard to wildlife. The second step, which is the aim of this report, provides decision makers with an objective and defensible method of assessing the risk associated with the pathogen. The third step is the implementation of risk management actions to mitigate the identified risk. Communication occurs throughout the process.

Uncertainty is the central challenge of risk management for decision makers - how to estimate the uncertainty and how to understand risk tolerance in choosing an action in the face of uncertainty. A quantification of risk combines the uncertainty in the range of outcomes and some measure of the potential harm under those outcomes. By framing the mitigation decisions with wildlife management agencies, we identified the outcomes that agencies wanted to avoid (the objectives articulated in the "Decision Framework" section). The task described in this report was to estimate the probability of several early steps in the causal chains that lead to those undesirable outcomes. Because of uncertainties about the distribution of the SARS-CoV-2 pathogen in the human population, the likelihood of transmission to bats, and the probability of infection given exposure, we combined expert elicitation and a model for the number of infected bats to estimate the current risk of transmission and the potential level of mitigation provided by the expanded use of PPE for individuals that conduct work with bats. Here, we considered risk as the combined probability of an event happening (that is, the transmission of SARS-CoV-2 from an infected human to a bat) and the magnitude of the undesired outcome (that is, persistence of the coronavirus within bat populations). The level of risk mitigated by a management intervention is determined by the reduction in likelihood of an undesired event and in the reduction of magnitude of the effect of the undesired event. Our aim was to provide decision makers with a risk assessment that could inform their risk management decisions in the near term, for activities that could affect bats over the next 6 months (April-November 2020).

\section{Summary of Results}

Our analysis finds a non-negligible risk of transmission of SARS-CoV-2 to bats from humans conducting research, survey, management, rehabilitation, and wildlife control activities. For example, our expert panel estimated that if a research scientist is shedding virus while handling bats under status quo protocols, 50 percent (15-84 percent) of those bats will be exposed to virus, and 17 percent (3-51 percent) will become infected. Although there were differences in exposure potential among the three transmission pathways (RSM, WR, and WC) and the three encounter types, without additional protective measures the probability of transmission resulting in infected bats cannot be ruled out. We found that the type of work being conducted changed the underlying risk; as expected, conducting work in proximity to bats (but in an unenclosed space) had a much lower risk of exposure than direct handling of bats.

We found that the use of PPE is expected to significantly reduce the exposure probabilities for all three modes of encounter with bats. The expert panel estimated that exposure risk from research scientists could be reduced by $94-96$ percent (uncertainty, 86-99 percent) through proper use of appropriate N95 respirators and dedicated clothing and gloves.

Finally, we estimate that the median likelihood of bat-to-bat transmission is lower than the value for sustained transmission (that is, the median $R_{0}$ value is less than 1.0). However, there was significant uncertainty for this rate, and there remains a reasonable probability (approximately 33 percent) that sustained bat-to-bat transmission will be possible should SARS-CoV-2 be introduced into a wild free-ranging bat population. Further research to better understand bat-to-bat SARS-CoV-2 transmission is warranted.

\section{Scope of Inference}

There are some potential limitations to our assessment. We used the little brown bat as a surrogate species for all North American bats for this risk assessment. The little brown bat is one of the most widespread species of Myotis, a genus which is diverse and widespread in North America (O'Shea and Bogan, 2003). This species frequently inhabits buildings (Fenton and Barclay, 1980; Kunz and Reynolds, 2003) and is commonly captured for scientific field work (for example, Frick and others, 2010), which leads to a potential for virus transmission from humans to bats. We asked the expert panel to think specifically of little brown bats while they were responding to the elicitation. Our ability to extend the conclusions of this study to other North American species of 
bats depends on the similarity of behavior, ecology, physiology, cellular biology, and genetics of those species to little brown bats.

The scope of inference for this analysis was limited to the active period for little brown bats, which we defined as the period from April 15 (when the bats leave hibernacula for maternal roost sites) through November 15 (when individuals return to hibernacula). This is when we expected the greatest contact rates with humans to occur. Because the active season for bat research, wildlife rehabilitation, and wildlife control activities was imminent, our assessment was designed to provide information to management agencies to update interim guidance to these groups. The expert panel and the modeling included herein focused on the active season. It is reasonable to think that the dynamics of exposure, infection, and subsequent transmission may be different during the hibernation season. Subsequent work may be needed to assess the risks for winter bat field work.

For PPE, we considered proper training, oversight, and use of dedicated clothing, gloves, and N95-type respirators. We expected use of PPE to have the largest effect on reducing bat exposure probability. In the elicitation, we did not make a distinction between vented and non-vented respirators, but from the discussion we assumed the experts were thinking about N95 respirators designed to filter exhaled particles. We did not specifically ask the expert panel about the use of alternative face coverings (like surgical masks), nor did we ask the experts to estimate the difference in efficacy of PPE among RSM, WR, and WC workers. In an open-ended question, some members of the expert panel did expect that proper and consistent use of appropriate N95 respirators might vary among groups. Note that other face coverings, including surgical masks, may be effective for reducing the transmission of SARS-CoV-2 because they have been found to be effective for human coronaviruses (Leung and others, 2020), but we do not know whether they will mitigate the transmission risk to the same level as expected for N95 respirators.

\section{Key Assumptions}

The inference in this report extends to regions with populations of little brown bats. The analysis contains an assumption that the number and modes of exposure reported by the 7 states, 3 agencies, and the WNS surveillance program represent the proportional encounter rates across the range of the little brown bat. Estimates of human exposure, shedding duration, and the basic reproduction number for SARS-CoV-2 in humans are all currently uncertain. We used estimates of cumulative incidence in humans from the emerging literature, assuming that the cumulative incidence over 7 months (April-November 2020) will be between 0.17 and 0.71 with 80-percent confidence. The forecasts of Moghadas and others (2020) that form the upper end of this interval assume no selfisolation of symptomatic individuals and an infectious period of 4.6 days, although their forecasts including self-isolation have little effect on reducing the epidemic size and peak timing. We thus assume that the current practices of stay-at-home, quarantine, and social distancing may reduce the duration of the epidemic but do not affect the cumulative incidence; given the current uncertainty in the ability to enforce or maintain such social distancing we believe this to be a reasonable baseline estimate to include in the risk assessment. Further, the reported cases do not include the asymptomatic individuals, and the estimated reporting rate (Bommer and Vollmer, 2020) is for actual cases attributed to COVID-19, so if the (adjusted) reported cases represent approximately 50 percent of the total infections (including mildly symptomatic and asymptomatic individuals; Nishiura and others, 2020; Li and others, 2020; World Health Organization, 2020; Ferguson and others, 2020; Mizumoto and others, 2020), then we believe this estimate to be reasonable for this risk assessment. We note that the asymptomatic portion of the population is a critical uncertainty, discussed in the section "Critical Uncertainties." The experts were asked to assume that a worker who is infected (positive for SARS-CoV-2) is not showing symptoms. We used an estimated median shedding duration of 33 days - asymptomatic individuals would shed virus for the entire period and would still contribute to bat exposure; we assumed that symptomatic individuals would cease work within 1 day of symptom onset, and would not return to work until 14 days after symptoms resolved. This means that symptomatic individuals would expose bats for 3-4 days before stopping work because they are infectious before symptom onset (He and others 2020).

Experts were asked about their belief in the compliance of the different groups for proper use of PPE. We assume for this analysis that the average effect of PPE was identical across all individuals regardless of profession. Experts were uncertain whether the proportional change in the handling and proximity exposure probabilities for wildlife rehabilitators and wildlife control operators, owing to the same protocol guidance, would be different than for scientists.

\section{Critical Uncertainties}

Critical uncertainties are those uncertainties which, if resolved, would change the selection of a mitigation strategy. Because we framed this problem as a decision-identifying the management agencies, authorities, objectives, and potential interventions, and developing a model to link actions with the risk of bat infection - we can identify those uncertainties in the analysis that we would expect to change the risk assessment. At this stage, we have not conducted a value-of-information analysis (Runge and others, 2011) to verify that these uncertainties are critical to the decisions, in part because we have not yet evaluated the full causal pathways. Instead, we discuss below (1) uncertainties we evaluated, which strongly affected the probabilities of the early steps in the causal chains, and (2) uncertainties we have not yet evaluated, but which we judge could have a strong effect on the long-term outcomes and bear future examination. 
Bat infection probability and sustained bat-to-bat transmission. These rates are related, and the probability a North American bat could become infected with SARS-CoV-2 was a significant uncertainty. Bats are known to maintain coronavirus infections, and experts expressed the opinion that North American bats were undertested for coronaviruses. New World bats from the Americas are known to host $\alpha$-coronaviruses (Osborne and others, 2011; Dominguez and others, 2007), but $\beta$-coronaviruses may be relatively rare (Anthony and others, 2013; Góes and others, 2013). For the infection rate, $\sigma_{M L}$, there is some evidence that the probability of infection may be low (based on sequence matching of the ACE2 receptor; Damas and others, 2020; Luan and others, 2020) but possible; at this time we do not have results from experimental virus challenge trials in bats that would provide the most useful information. SARS-CoV-2 is a member of a group of viruses that is prone to host switching and recombination (Woo and others 2009), so transmission among species may be possible if it establishes in a population of a single species.

Species and regional differences. We also considered in our discussions Eptesicus fuscus (big brown bats) and Tadarida brasiliensis (Brazilian free-tailed bats), which also have widespread distributions but may have different human contact rates, infection probabilities, basic reproduction numbers, and transmission rates. The important questions to ask in extending this analysis to other species will be which parameters might differ and whether those differences might affect the baseline risk or the effect of proposed mitigation strategies. Species, communities, roost sites, availability, adherence to PPE guidelines, and the modes of interaction between bats and humans may all differ regionally.

The human probability of infection, shedding, and the amount of viral particles shed. The probability of transmission from humans to bats increases with both the duration of exposure and the quantity of viral particles shed. Shedding of viral particles changes over the course of infection and may peak even before symptoms manifest (He and others, 2020). It is unknown whether asymptomatic infected individuals shed virus in the same manner (duration and amount) as symptomatic individuals.

The asymptomatic frequency and shedding rate. A key uncertainty in estimating the exposure risk is the number of truly infected people that will encounter bats. We assumed for this analysis that individuals who are symptomatic are not conducting work with or near bats. Emerging evidence indicates that individuals may be infected with SARS-CoV-2 without showing symptoms. Estimates of the fraction of infections that are asymptomatic range from an average of 30.8 percent (Nishiura and others, 2020) to 86 percent (Li and others, 2020; also see World Health Organization, 2020; Ferguson and others, 2020; Mizumoto and others, 2020).

General public as a source of SARS-CoV-2. We did not estimate the potential transmission from the public at large to bats. Bats in the living spaces of houses may constitute a large fraction of the submissions for rabies testing, and it is estimated that many bats that are exposed to human spaces are released by the public rather than sent for testing. Further, people may not be aware of bats in their attics or barns and may expose bats to aerosols containing SARS-CoV-2. We assumed that contact from wildlife researchers, surveyors, managers, rehabilitators, and control operators would occur only when individuals were asymptomatic, but people recovering from infection in their homes may also expose bats roosting or trapped in homes.

\section{Future Steps}

From a rapid initial risk assessment associated with a subset of the parameters in the full influence diagram (fig. 1), we aim to provide information to decision makers that they can apply in their specific settings. Ultimately, how agencies use this decision framing and risk assessment may differ across agencies, taking into account their specific mandates. Different decision makers may tolerate varying amounts of risk (that is, agencies may have different acceptable levels of protection) and, thus, may choose to implement different sets of mitigation actions.

Future work may take several forms. First, we may improve upon the risk assessment presented here with updated parameter estimates from empirical data. There is ongoing research of human and bat systems that can update our parameter estimates (for example, challenge trials for the $R_{0}$ and $\sigma$ parameters for bats, and the components of the $p_{R S M}^{+}$parameter in humans). Second, we considered probability of exposure and infection for the active field season only; different kinds of work, with potentially different exposure and infection risks, are conducted during the winter hibernation period. Guidelines developed from this risk analysis for wildlife scientists, rehabilitators, and control operators may differ for winter work. Third, we focused on a subset of State and Federal agencies. We may expand the scope of future assessments to include a larger number of State, Federal, and tribal agencies. Fourth, we focused only on the initial transmission stages (fig. 2) and did not evaluate the remaining steps in the causal diagram (fig. 1). Work on the remainder of the system diagram would include re-transmission to humans, domesticated animals, and other wildlife, and may reveal other management actions that could reduce the risk to the full set of objectives that are important to management agencies. In addition, there are more complex dynamics within the causal diagram that might be important to study, like the interplay between WNS and coronavirus shedding in bats (Davy and others, 2018). Fifth, we consider risks to bat populations from human exposure, but there are other human-animal interactions that may present risk to wildlife populations. A node was included in the influence diagram for feral and domestic cat infection because there is increasing evidence that felids may sustain infections (Shi and others, 2020). Given that feral and free-ranging cats already pose a significant risk to wildlife populations, including zoonotic disease (Medina and others, 2014), it may be important to expand the influence diagram and estimate the probabilities along this exposure route. 


\section{Summary}

This report describes a risk assessment led by the U.S. Geological Survey, in cooperation with the U.S. Fish and Wildlife Service, to examine the possibility of reverse zoonotic transmission of SARS-CoV-2, the coronavirus that causes the human disease COVID-19, from humans to bats in North America. The study was undertaken to inform State, Federal, and tribal wildlife management agencies in the United States that manage some aspects of the interactions between humans and bats and are in the process of developing guidance.

The study was designed by first framing the decisions that concern State and Federal wildlife agencies, with a focus on the long-term outcomes the agencies desire and the near-term mitigation actions that are within their authority to implement. From these components, a causal diagram was developed to trace the linkages from the potential actions through intermediate steps to the desired outcomes. The subsequent risk analysis focused on the early steps of three transmission pathways, namely the exposure and infection of bats from research, survey, monitoring, and management activities; wildlife rehabilitation; and nuisance wildlife control operations. The assessment focused on the immediate field season, from April to November 2020, and used little brown bats as a case study and as a surrogate species for other North American bats.

From the causal diagram, a quantitative model was developed to forecast the number of infected bats using estimates of the number of bats handled through the three transmission pathways, the probability of exposure through various modes of encounter, the probability of infection conditional on exposure, and the probability that a worker is actively shedding the SARS-CoV-2 virus. The parameters in this model were estimated primarily through a formal process of expert judgment.

The expert panel estimated that, if a research scientist were shedding virus while handling bats under the protocols in use prior to the COVID-19 pandemic, 50 percent (15-84 percent) of those bats would be exposed to virus, and 17 percent (3-51 percent) would become infected. Although there were differences in exposure potential among the transmission pathways and encounter types, without additional protective measures, the probability of transmission resulting in infected bats cannot be ruled out. The expert panel expected that proper use of personal protective equipment would significantly reduce the exposure probabilities for all modes of encounter with bats. For example, the panel estimated that exposure risk from research scientists could be reduced by $94-96$ percent (uncertainty, 86-99 percent) through proper use of appropriate personal protective equipment (such as N95 respirators and dedicated clothing and gloves). Regarding the possibility of sustained bat-to-bat transmission of SARS-CoV-2 in a wild population of little brown bats, the expert panel estimated a median basic reproduction number $\left(R_{0}\right)$ of 0.45 , but expressed considerable uncertainty, such that there was a 33-percent chance that $R_{0}$ could be greater than 1 ; these results indicate that sustained transmission within bat populations is a possibility, if SARS-CoV-2 is introduced.

This research was conducted at a time when there were few empirical studies of SARS-CoV-2 in North American bats, so there was considerable uncertainty in the results. As new information becomes available, the risk model can be updated with data concerning human infection rates, bat infection rates, and other parameters. Future work could look at the risks posed by field work after November 2020 (that is, during the hibernation season), as well as later steps in the causal diagram.

\section{References Cited}

Anthony, S.J., Johnson, C.K., Greig, D.J., Kramer, S., Che, X., Wells, H., Hicks, A.L., Joly, D.O., Wolfe, N.D., Daszak, P., Karesh, W., Lipkin, W.I., Morse, S.S., PREDICT Consortium, Mazet, J.A.K., and Goldstein, T., 2017, Global patterns in coronavirus diversity: Virus Evolution, v. 3, no. 1, vex012, accessed May 19, 2020, at https://doi.org/10.1093/ ve/vex012.

Anthony, S.J., Ojeda-Flores, R., Rico-Chávez, O., NavarreteMacias, I., Zambrana-Torrelio, C.M., Rostal, M.K., Epstein, J.H., Tipps, T., Liang, E., Sanchez-Leon, M., SotomayorBonilla, J., Aguirre, A.A., Ávila-Flores, R., Medellín, R.A., Goldstein, T., Suzán, G., Daszak, P., and Lipkin, W.I., 2013, Coronaviruses in bats from Mexico: The Journal of General Virology, v. 94, no. 5, p. 1028-1038, accessed May 19, 2020, at https://doi.org/10.1099/vir.0.049759-0.

Bommer, C., and Vollmer, S., 2020, Average detection rate of SARS-CoV-2 infections has improved since our last estimates but is still as low as nine percent on March 30th: Georg-August-Universität Göttingen, accessed May 4, 2020, at http://www.uni-goetti gen.de/de/document/downloa d/0af0dcfa623053908de337e1045cf612.pdf/COVID-19\%20 update.pdf.

Cooke, R.M., 1991, Experts in uncertainty-opinion and subjective probability in science: Oxford, UK, Oxford University Press., 321 p.

Damas, J., Hughes, G.M., Keough, K.C., Painter, C.A., Persky, N.S., Corbo, M., Hiller, M., Koepfli, K.-P., Pfenning, A.R., Zhao, H., Genereux, D.P., Swofford, R., Pollard, K.S., Ryder, O.A., and Nweeia, M.T., 2020, Broad host range of SARS-CoV-2 predicted by comparative and structural analysis of ACE2 in vertebrates: bioRxiv, 2020.2004.2016.045302, accessed May 4, 2020, at https:// doi.org/10.1101/2020.04.16.045302. 
Davy, C.M., Donaldson, M.E., Subudhi, S., Rapin, N., Warnecke, L., Turner, J.M., Bollinger, T.K., Kyle, C.J., Dorville, N.A.S.-Y., Kunkel, E.L., Norquay, K.J.O., Dzal, Y.A., Willis, C.K.R., and Misra, V., 2018, White-nose syndrome is associated with increased replication of a naturally persisting coronaviruses in bats: Scientific Reports, v. 8, art. 15508(2018), accessed May 19, 2020, at https://doi. org/10.1038/s41598-018-33975-x.

Dominguez, S.R., O’Shea, T.J., Oko, L.M., and Holmes, K.V., 2007, Detection of group 1 coronaviruses in bats in North America: Emerging Infectious Diseases, v. 13, no. 9, p. 1295-1300, accessed May 19, 2020, at https://doi. org/10.3201/eid1309.070491.

Fenton, M.B., and Barclay, R.M.R., 1980, Myotis lucifugus: Mammalian Species, v. 142, no. 142, p. 1-8, accessed May 19, 2020, at https://doi.org/10.2307/3503792.

Ferguson, N.M., Laydon, D., Nedjati Gilani, G., Imai, N., Ainslie, K., Baguelin, M., Bhatia, S., Boonyasiri, A., Cucunuba Perez, Z., Cuomo-Dannenburg, G., Dighe, A., Dorigatti, I., Fun, H., and Gaythorpe, K., 2020, Impact of non-pharmaceutical interventions (NPIs) to reduce COVID-19 mortality and healthcare demand: London, UK, Imperial College London, Report 9 (March 16, 2020), accessed May 19, 2020, at https://doi.org/10.25561/77482.

Frick, W.F., Pollock, J.F., Hicks, A.C., Langwig, K.E., Reynolds, D.S., Turner, G.G., Butchkoski, C.M., and Kunz, T.H., 2010, An emerging disease causes regional population collapse of a common North American bat species: Science, v. 329, no. 5992, p. 679-682, accessed May 19, 2020, at https://doi.org/10.1126/science.1188594.

Góes, L.G.B., Campos, A.C. de A., Carvalho, C. de, Ambar, G., Queiroz, L.H., Cruz-Neto, A.P., Munir, M., and Durigon, E.L., 2016, Genetic diversity of bats coronaviruses in the Atlantic Forest hotspot biome, Brazil: Infection, Genetics and Evolution, v. 44, p. 510-513, accessed May 19, 2020, at https://doi.org/10.1016/j.meegid.2016.07.034.

Góes, L.G.B., Ruvalcaba, S.G., Campos, A.A., Queiroz, L.H., Carvalho, C. de, Jerez, J.A., Durigon, E.L., Dávalos, L.I.I., and Dominguez, S.R., 2013, Novel bat coronaviruses, Brazil and Mexico: Emerging Infectious Diseases, v. 19, no. 10, p. 1711-1713, accessed May 19, 2020, at https://doi. org/10.3201/eid1910.130525.

Hanea, A., McBride, M., Burgman, M., Wintle, B., Fidler, F., Flander, L., Twardy, C., Manning, B., and Mascaro, S., 2017, Investigate Discuss Estimate Aggregate for structured expert judgement: International Journal of Forecasting, v. 33, no. 1, p. 267-279, accessed May 19, 2020, at https:// doi.org/10.1016/j.ijforecast.2016.02.008.
He, X., Lau, E.H., Wu, P., Deng, X., Wang, J., Hao, X., Lau, Y.C., Wong, J.Y., Guan, Y., Tan, X., Mo, X., and Chen, Y., 2020, Temporal dynamics in viral shedding and transmissibility of COVID-19: Nature Medicine, v. 26, no. 5, p. 672-675, accessed May 19, 2020, at https://doi. org/10.1038/s41591-020-0869-5.

Kullback, S., and Leibler, R.A., 1951, On information and sufficiency: Annals of Mathematical Statistics, v. 22, no. 1, p. 79-86, accessed May 19, 2020, at https://doi.org/10.1214/ aoms/1177729694.

Kunz, T.H., and Reynolds, D.S., 2003, Bat colonies in buildings, in O'Shea, T.J., and Bogan, M.A., eds., Monitoring trends in bat populations of the United States and territories_-problems and prospects: U.S. Geological Survey Information and Technology Report USGS/BRD/ITR 20030003, p. 91-102.

Leung, N.H.L., Chu, D.K.W., Shiu, E.Y.C., Chan, K.-H., McDevitt, J.J., Hau, B.J.P., Yen, H.-L., Li, Y., Ip, D.K.M., Peiris, J.S.M., Seto, W.-H., Leung, G.M., Milton, D.K., and Cowling, B.J., 2020, Respiratory virus shedding in exhaled breath and efficacy of face masks: Nature Medicine, v. 26, no. 5, p. 676-680, accessed May 19, 2020, at https://doi. org/10.1038/s41591-020-0843-2.

Li, R., Pei, S., Chen, B., Song, Y., Zhang, T., Yang, W., and Shaman, J., 2020, Substantial undocumented infection facilitates the rapid dissemination of novel coronavirus (SARS-CoV-2): Science, v. 368, no. 6490, p. 489-493, accessed May 19, 2020, at https://doi.org/10.1126/science. abb3221.

Luan, J., Jin, X., Lu, Y., and Zhang, L., 2020, SARS-CoV-2 spike protein favors ACE2 from Bovidae and Cricetidae: Journal of Medical Virology, accessed May 19, 2020, at https://doi.org/10.1002/jmv.25817.

Medina, F.M., Bonnaud, E., Vidal, E., and Nogales, M., 2014, Underlying impacts of invasive cats on islands-Not only a question of predation: Biodiversity and Conservation, v. 23, no. 2, p. 327-342, accessed May 19, 2020, at https://doi. org/10.1007/s10531-013-0603-4.

Mizumoto, K., Kagaya, K., Zarebski, A., and Chowell, G., 2020, Estimating the asymptomatic proportion of coronavirus disease 2019 (COVID-19) cases on board the Diamond Princess cruise ship, Yokohama, Japan, 2020: Eurosurveillance, v. 25, no. 10, p. 2000180, accessed May 19, 2020, at https://doi.org/10.2807/1560-7917.ES.2020.25.10.2000180. 
Moghadas, S.M., Shoukat, A., Fitzpatrick, M.C., Wells, C.R., Sah, P., Pandey, A., Sachs, J.D., Wang, Z., Meyers, L.A., Singer, B.H., and Galvani, A.P., 2020, Projecting hospital utilization during the COVID-19 outbreaks in the United States: Proceedings of the National Academy of Sciences of the United States of America, v. 117, no. 16, p. 9122-9126, accessed May 19, 2020, at https://doi.org/10.1073/ pnas. 2004064117.

Nishiura, H., Kobayashi, T., Miyama, T., Suzuki, A., Jung, S., Hayashi, K., Kinoshita, R., Yang, Y., Yuan, B., Akhmetzhanov, A.R., and Linton, N.M., 2020, Estimation of the asymptomatic ratio of novel coronavirus infections (COVID-19): International Journal of Infectious Diseases, v. 94, p. 154-155, accessed May 19, 2020, at https://doi. org/10.1016/j.ijid.2020.03.020.

O'Shea, T.J., and Bogan, M.A., eds., 2003, Monitoring trends in bat populations of the United States and territoriesproblems and prospects: U.S. Geological Survey Information and Technology Report USGS/BRD/ITR 2003-0003, $274 \mathrm{p}$.

O’Shea, T.J., Cryan, P., and Bogan, M.A., 2018, United States bat species of concern: a synthesis: Proceedings of the California Academy of Sciences, 4th series, v. 65, no. Supplement 1, p. 1-279.

Osborne, C., Cryan, P.M., O'Shea, T.J., Oko, L.M., Ndaluka, C., Calisher, C.H., Berglund, A.D., Klavetter, M.L., Bowen, R.A., Holmes, K.V., and Dominguez, S.R., 2011, Alphacoronaviruses in New World bats-Prevalence, persistence, phylogeny, and potential for interaction with humans: PLoS One, v. 6, no. 5, p. e19156, accessed May 19, 2020, at https://doi.org/10.1371/journal.pone.0019156.

Pieracci, E.G., Brown, J.A., Bergman, D.L., Gilbert, A., Wallace, R.M., Blanton, J.D., Velasco-Villa, A., Morgan, C.N., Lindquist, S., and Chipman, R.B., 2020, Evaluation of species identification and rabies virus characterization among bat rabies cases in the United States: Journal of the American Veterinary Medical Association, v. 256, no. 1, p. 77-84, accessed May 19, 2020, at https://doi.org/10.2460/ javma.256.1.77.

Runge, M.C., Converse, S.J., and Lyons, J.E., 2011, Which uncertainty? Using expert elicitation and expected value of information to design an adaptive program: Biological Conservation, v. 144, no. 4, p. 1214-1223, accessed May 19, 2020, at https://doi.org/10.1016/j.biocon.2010.12.020.

Shi, J., Wen, Z., Zhong, G., Yang, H., Wang, C., Huang, B., Liu, R., He, X., Shuai, L., Sun, Z., Zhao, Y., Lui, P., Liang, L., Cui, P., Wang, J., Zhang, X., Guan, Y., Tan, W., Wu, G., Chen, H., and Bu, Z., 2020, Susceptibility of ferrets, cats, dogs, and other domesticated animals to SARS-coronavirus 2: Science, p. abb7015, accessed May 19, 2020, at https://doi.org/10.1126/science.abb7015.
Speirs-Bridge, A., Fidler, F., McBride, M.F., Flander, L., Cumming, G., and Burgman, M.A., 2010, Reducing overconfidence in the interval judgments of experts: Risk Analysis, v. 30, no. 3, p. 512-523, accessed May 19, 2020, at https:// doi.org/10.1111/j.1539-6924.2009.01337.x.

Wölfel, R., Corman, V.M., Guggemos, W., Seilmaier, M., Zange, S., Müller, M.A., Niemeyer, D., Jones, T.C., Vollmar, P., Rothe, C., Hoelscher, M., and Bleicker, T., 2020, Virological assessment of hospitalized patients with COVID-2019: Nature, accessed May 19, 2020, at https:// doi.org/10.1038/s41586-020-2196-x.

Woo, P.C., Lau, S.K., Huang, Y., and Yuen, K.Y., 2009, Coronavirus diversity, phylogeny and interspecies jumping: Experimental Biology and Medicine, v. 234, no. 10, p. 1117-1127, accessed May 19, 2020, at https://doi. org/10.3181/0903-MR-94.

World Health Organization, 2020, Coronavirus disease 2019 (COVID-19): Geneva, Switzerland, World Health Organization Situation Report-46, 9 p.

World Organisation for Animal Health, 2019, OIE Aquatic Animal Health Code (22d ed.): Paris, France, World Organisation for Animal Health, accessed May 4, 2020, at https://www.oie.int/en/standard-setting/aquatic-code/accessonline/.

Zhou, B., She, J., Wang, Y., and Ma, X., 2020a, The duration of viral shedding of discharged patients with severe COVID-19: Clinical Infectious Diseases, ciaa451, accessed May 19, 2020, at https://doi.org/10.1093/cid/ciaa451.

Zhou, P., Yang, X.-L., Wang, X.-G., Hu, B., Zhang, L., Zhang, W., Si, H.-R., Zhu, Y., Li, B., Huang, C.-L., Chen, H.-D., Chen, J., Luo, Y., Guo, H., Jiang, R.-D., Liu, M.-Q., Chen, Y., Shen, X.-R., Wang, X., Zheng, X.-S., Zhao, K., Chen, Q.-J., Deng, F., Liu, L.-L., Yan, B., Zhan, F.-X., Wang, Y.-Y., Xiao, G.-F., and Shi, Z.-L., 2020b, A pneumonia outbreak associated with a new coronavirus of probable bat origin: Nature, v. 579 , no. 7798 , p. 270-273, accessed May 19, 2020, at https://doi.org/10.1038/s41586-020-2012-7.

Zou, L., Ruan, F., Huang, M., Liang, L., Huang, H., Hong, Z., Yu, J., Kang, M., Song, Y., Xia, J., Guo, Q., Song, T., He, J., Yen, H.-L., Peiris, M., and Wu, J., 2020, SARSCoV-2 viral load in upper respiratory specimens of infected patients: The New England Journal of Medicine, v. 382, no. 12, p. 1177-1179, accessed May 19, 2020, at https://doi. org/10.1056/NEJMc2001737. 


\section{Appendix 1. Instructions for the Expert Panel}

This appendix contains that exact documents that were provided to the experts during the expert elicitation process. The contents of this Appendix have not been edited to conform to USGS editorial standards. 


\section{Introduction to Expert Elicitation for an Expert Panel}

\section{Justification for an Expert Elicitation}

Ideally, we would obtain parameter estimates from empirical data and associated mathematical models. Because these information are unavailable and time is of the essence for decision makers, we aim to use an expert panel to elicit parameter values with associated uncertainty, using techniques of expert judgment that utilize best available scientific information, account for uncertainty, and reduce bias (Morgan, 2014; Sutherland and Burgman, 2015).

Expert elicitation is a formal, structured process of obtaining expert judgment for specific questions. An expert is someone who possesses substantive information on a particular topic that is not widely known by others. We know that experts have knowledge, often privileged knowledge, that accrues as a result of their research and experience, even about processes for which data have not been collected. The question is how to extract that knowledge accurately and precisely. Expert judgment is a quantitative expression of an expert's belief based on knowledge and experience; it is an informed belief. Expert elicitation can provide improved information over single-expert inquiry when a diverse group of experts is asked to provide estimates, using a facilitated approach with discrete opportunities for information sharing, provision of estimates, and review of summarized information (Martin and others, 2012). Expert elicitation, when conducted with the same level of rigor as the collection and use of empirical data, can result in reliable predictions (for example, O'Hagan and others, 2006; Speirs-Bridge and others, 2010; Runge and others, 2011; Martin and others, 2012; Adams-Hosking and others, 2016).

An expert elicitation is governed by specific protocols to avoid inherent biases resulting from cognitive traps. These cognitive traps are shortcuts, or heuristics, that serve us well for simple decisions but result in biased estimates for more complex tasks (O'Hagan, 2019). These biases include

- Availability bias (experts will be influenced by evidence or events that are easily recalled),

- Anchoring bias (experts fail to consider possible values far from an initial estimate),

- Overconfidence (experts tend to underestimate their uncertainty, and make forecasts that are too narrow),

- Representativeness bias (a tendency to think of probabilities related to readily available examples), and
- Motivational bias (an innate desire to further our own interests).

When the number of experts is limited, we would additionally be concerned about small-sample bias.

There are additional biases that arise through the behavior of groups. To some extent, these can be collectively referred to as "groupthink," the tendency for groups to converge too quickly on consensus estimates or decisions and to ignore or forget divergent views that are held by members of the group. In this way, groups of experts can be collectively overconfident, or even biased.

So, the methodological challenge of expert judgment is to reliably extract the desired information from each member of a group of experts, without falling into the cognitive and behavioral biases that can undermine such an exercise. The best practices in an expert judgment approach have evolved by considering this challenge, testing approaches via experiments, and recommending a set of protocols for conducting an expert elicitation.

\section{Steps in an Elicitation}

We are using a protocol based on a modified Delphi method called the IDEA protocol (Hanea and others, 2017), with the four-point elicitation method (Speirs-Bridge and others, 2010). There are six steps in the process:

1. Select experts;

2. Calibrate experts (seed questions and sharing available information);

3. Elicitation of parameter values (4-point method);

4. Summary, review, and discussion (aimed at reducing linguistic uncertainty - relating to the instructions - and sharing insights, not to reach consensus);

5. Experts revise their initial values (if desired); and

6. Aggregate information across experts.

Steps 3-6 comprise a modified Delphi approach (described below).

\section{Selection of Experts}

Experts are individuals with specific subject-matter experience and knowledge. Experts should have relevant expertise which may come from formal training and be demonstrated by professional accomplishments such as peer-reviewed publications, familiarity with and knowledge of the system or related systems, willingness to participate fully and impartially in an 
elicitation process, and good interpersonal and communication skills (Ayyub, 2001; Fazey and others, 2006).

Groups of experts have been found to perform as well (in terms of providing information close to the true empirically observed data) as more specific experts (for example, Burgman and others, 2011). The expert panel should be diversepossessing knowledge of North American bats, zoonotic disease, and possible mitigation strategies; representing multiple institutions, specialization, and gender. The optimal number of experts for a structured elicitation is between 5 and 12, with decreasing marginal benefit after 12 experts (Hogarth, 1978; Hemming and others, 2018).

\section{Training Questions}

Before starting the elicitation concerning the questions of interest, we will provide the expert panel a chance to practice the elicitation methods. We will provide questions that are known (that is, we have identified values from the literature, but are unlikely to be known precisely by experts). We use these questions to ensure that the instructions are understood by experts, and to allow experts a chance to calibrate their estimates of uncertainty.

Three questions are listed below (see accompanying spreadsheet $<$ BatEE Practice Questions v2.xlsx $>$ [not included with this report]). For each question, we ask experts to provide four responses: an estimate that represents your view of the lowest reasonable value; an estimate of the highest reasonable value; an estimate that represents the best central value; and your confidence that the true value lies within the low and high values that you have provided. We have attached a spreadsheet in which you can enter these values; the spreadsheet automatically calculates a probability distribution that represents your uncertainty, as immediate feedback about whether your responses reflect your expert belief. This is a "closed book" exercise (we ask that you do not check this information in books or online). Please return your answers to us; we will use them to provide feedback to the group about your individual and collective accuracy and precision; as a means of allowing you to calibrate your thinking process prior to the elicitation for the questions of central importance.

The calibration questions are

1. What is the mean forearm length (in centimeters) of an adult little brown bat (Myotis lucifugus)?

2. What is the average number of subsequent white-nose syndrome infections resulting from a single infected little brown bat (that is, $R_{0}$ )?

3. In a population that has already experienced decline due to WNS, out of 100 adult female little brown bats, how many would you expect to breed in a given year?

\section{Elicitation of Parameters Using a Modified Delphi Approach}

To generate empirical estimates of each parameter, we use a "4-point" elicitation method. This approach has been shown to reduce overconfidence in experts (Speirs-Bridge and others, 2010) and can generate a quantitative estimate from experts who may be uncomfortable providing estimates. We derive a median and credible interval for each parameter from the following four questions:

1. Realistically, what is the lowest reasonable value for the parameter?

2. Realistically, what is the highest reasonable value for the parameter?

3. Realistically, what is the most likely reasonable value (that is, your best estimate) for the parameter?

4. How confident are you that the true value is between the lowest and highest values you provided?

We then assume that the most likely value is the median value, and combine the upper and lower estimates and the reported confidence to generate a credible interval.

Experts provide their estimates anonymously, and summaries are provided that maintain anonymity, to avoid biases associated with group thinking and dominant personalities. Experts are encouraged to discuss the information during a facilitated discussion of the summarized data, after which experts have the opportunity to revise any of their estimates.

The modified Delphi sequence (independent-group-independent) is important to preserve the unique insights held by individuals while at the same time allowing the benefit of wisdom to be shared. By asking experts to perform the first estimate independently, their own personal views are captured. By allowing the expert to share and discuss their initial estimates, we can explore whether there is residual linguistic uncertainty that needs to be corrected and we can allow insights to be shared across experts. By allowing the final estimates to be made independently, we guard against dominant voices in the group and retain the diversity of insights among the experts.

\section{Aggregation of Information Across Experts}

Following the elicitation, we will aggregate the results to produce a single probability distribution that represents an estimate, with uncertainty, for each parameter. To do this, we will first transform the four-point elicitation results into a probability distribution for each expert. We will then average these probability distributions across experts, with equal weighting. (There are involved methods for weighting experts based on sets of calibration questions, but we are both skeptical of these methods and limited on time). 


\section{Questions for the Expert Panel}

For each of the questions that ask for a quantitative response, we are asking you to provide a low estimate, a high estimate, a central estimate, and a degree of confidence that the true value is between your low and high estimates. Please see the document that provides instructions on expert elicitation that was sent by Evan Grant on April 9 [see "Introduction to Expert Elicitation for an Expert Panel"]. Please record your responses in the accompanying spreadsheet, which also provides graphical feedback.

In all the questions below, unless otherwise noted, we are thinking specifically about little brown bats (Myotis lucifugus) throughout their range in North America, with a focus on the time period between now and the initiation of hibernation in the autumn of 2020.

Questions 1-7 all involve estimation of an exposure probability in the absence of any new restrictions, regulations, or protocols, that is, under the status quo conditions for contact with bats that existed before the arrival of SARS-CoV-2 in North America. In the past, concern about biological agents has been primarily focused on rabies virus and the fungus $P$. destructans; typical protocols involve decontamination of clothing and footwear between sites, wearing nitrile gloves (with disposal or decontamination between bats), but use of face masks or respirators has not been typical.

Note that we are separating the processes of exposure and infection. By exposure probability, we mean the likelihood that a particular interaction between an average bat and a biologist who is actively shedding SARS-CoV-2 virus will result in exposure of the bat to a sufficient viral dose to cause infection. By infection probability, we mean the probability that the virus replicates in the host (bat) tissue, conditional on that bat having been exposed to a sufficient viral dose. That is, the exposure process is about whether enough virus was transferred to make an infection possible; it is a property of the interaction between the biologist and that bat. The infection process is about the molecular, cellular, immunological, and physiological conditions that allow replication in the bat; it is a property of the interaction of the bat and the pathogen. Questions 1-7 only ask if the bat will be exposed to a sufficient viral dose; Question 8 asks about the probability of developing an infection, conditional on exposure.

Questions 1-7 differ from each other in two respects: the exposure pathway (the types of work being conducted), and the degree of interaction. We consider three exposure pathways: through activities related to research, survey, monitoring, and management (RSM); through wildlife rehabilitation (WR); and through wildlife control operations (WC). We consider three degrees of interaction: handling; proximity in an enclosed spaced without handling; and proximity in an unenclosed space without handling.

1. Consider a wildlife biologist engaged in research, survey, monitoring, or management (RSM) who is actively shedding SARS-CoV-2 virus $(\mathrm{CoV}+)$, performing their routine activities in the absence of any new restrictions, regulations, or protocols. If that biologist directly handles 100 average little brown bats, how many of those bats do you estimate will be exposed to a sufficient viral dose of SARS-CoV-2 that they could become infected? (This relates to the parameter $\beta_{H}^{R S M}$ in equation 1).

2. Same setting as question $1, \mathrm{a} \mathrm{CoV}+$ biologist conducting RSM under status quo protocols. If that biologist is in an enclosed space and within 6 feet of 100 average little brown bats (but does not handle them), how many of those bats will be exposed to a sufficient viral dose that they could become infected? (This relates to the parameter $\beta_{E}^{R S M}$ in equation 1 ).

3. Same setting as question 1. If the RSM biologist is not in an enclosed space but is within a 6-foot proximity of 100 little brown bats (and does not handle them), how many of those bats will be exposed to a sufficient viral dose that they could become infected? (This relates to the parameter $\beta_{P}^{R S M}$ in equation 1).

4. Now consider a wildlife rehabilitator (WR) who is actively shedding SARS-CoV-2 virus ( $\mathrm{CoV}+)$, performing their routine activities in the absence of any new restrictions, regulations, or protocols. If that rehabilitator directly handles 100 average little brown bats, how many of those bats do you estimate will be exposed to a sufficient viral dose of SARS-CoV-2 that they could become infected? (This relates to the parameter $\beta_{H}^{W R}$ in equation 2).

5. Same setting as question $4, \mathrm{a} \mathrm{CoV}+$ wildlife rehabilitator (WR) conducting their work under status quo protocols. If that rehabilitator is within a 6-foot proximity (whether enclosed or unenclosed) of 100 average little brown bats but does not handle them, how many of those bats will be exposed to a sufficient viral dose that they could become infected? (This relates to the parameter $\beta_{P}^{W R}$ in equation 2).

6. Now consider a wildlife control operator (WC) who is actively shedding SARS-CoV-2 virus $(\mathrm{CoV}+)$, performing their routine activities that involve handling bats, in the absence of any new restrictions, regulations, or protocols. For example, a typical activity might involve capturing bats in a home or trapping and transporting bats from an attic. If that WC operator directly handles 100 average little brown bats, how many of those bats do you estimate will be exposed to a sufficient viral dose of SARS-CoV-2 that they could become infected? (This relates to the parameter $\beta_{H}^{W C}$ in equation 3).

7. Same setting as question $6, \mathrm{a} \mathrm{CoV}+$ wildlife control operator (WC) conducting their routine work under status quo protocols, but without handling the bats. For example, a typical activity might involve working in an attic to set up an exclusion device, or trapping bats without handling 
them. If that WC operator is within a 6-foot proximity (whether enclosed or unenclosed) of 100 average little brown bats but does not handle them, how many of those bats will be exposed to a sufficient viral dose that they could become infected? (This relates to the parameter $\beta_{P}^{W C}$ in equation 3 ).

The next question focuses on the probability of infection, conditional on exposure. (This relates to the parameter $\sigma_{\mathrm{ML}}$ in equations 1,2 , and 3.)

8. What is the probability that a little brown bat exposed to a sufficient viral dose of SARS-CoV-2 would actually become infected by the virus (that is, sustained viral replication would occur in their tissue)?

The next three questions focus on the efficacy of guidance and protocols to reduce the exposure rate. In all of these questions, the new guidance and protocols consist of: restriction of fieldwork to people without symptoms and without contact with someone who had symptoms of COVID-19 in the last 14 days; proper training and compliance protocols for the use of PPE; proper use of Tyvek or other dedicated clothing; proper use of an N95 respirator; and proper use of gloves for handling bats. In these questions, please assume that the biologists have proper training, have access to PPE, and are using it appropriately.

9. Consider your response to question 1, regarding exposure through handling by RSM scientists. By what proportion should this exposure probability be multiplied if the new guidance and protocols are put into place? (Note that a proportion of 1 means there would be no change in exposure probability; a proportion of less than 1 would indicate a reduction in exposure probability; and a proportion of greater than 1 would indicate an increase in exposure probability as a result of such guidance.)

10. Consider your response to question 2 , regarding exposure through proximity in an enclosed space by RSM scientists. By what proportion should this exposure probability be multiplied if the new guidance and protocols are put into place?

11. Consider your response to question 3 , regarding exposure through proximity in an unenclosed space by RSM scientists. By what proportion should this exposure probability be multiplied if the new guidance and protocols are put into place?

12. Open-ended response. Are there reasons to believe that the proportional change in the handling and proximity exposure probabilities for wildlife rehabilitators (WR) and wildlife control operators (WC), owing to the same protocol guidance, would be different than for scientists involved in research, survey, and management (RSM)? Explain.
The last question addresses the risk of sustained bat-tobat transmission of SARS-CoV-2.

13. What is $R_{0}$ for SARS-CoV-2 in little brown bats during the active season? That is, for each infected little brown bat, how many other little brown bats would become infected with the virus? Note that $R_{0}$ can be less than 1 , in which case you can think of it as the probability that an infected bat will infect one other bat, or it can be greater than 1 , in which case each infected bat infects more than one other bat. Note that the spreadsheet [not included in this report] calculates from your responses the probability that $R_{0}$ is greater than 1 .

We are grateful for your time and expertise. Thank you for your thoughtful participation in this elicitation.

\section{Clarification Provided Between Rounds of Elicitation}

During the discussion with the experts between Rounds 1 and 2 of the elicitation, the experts raised some questions about the typical activities of RSM, WR, and WC workers when encountering bats. The following clarifications were provided before the second round of elicitation was completed.

Because research on bats typically involves more than one scientist, we consider the number encountered by each member of a research team; the $\beta$ parameters in equation 1 describe the exposure probability per scientist while conducting each of the activities. The description of typical handling procedures for researchers working with bats includes: 1-2 minutes of contact per bat, holding a bat within 12 inches of the face, taking morphometrics, and blowing on a bat to aid in determining reproductive condition or to discourage biting. Some research and management activities may involve longer holding periods for collection of metabolic measurements, attachment of radiotransmitters and other sampling, but these interactions are less common. The definition of enclosed space includes caves and mines with various sizes and morphologies that may result in variation in airflow among sites. We assumed that activity in enclosed space may be greater than 1 hour, and bats in these spaces may be a mixture of stationary (roosting) and in flight. Typical activities near bats but in an unenclosed space include a management agency conducting emergence counts outside a cave or mine entrance or under a bridge.

Typical activities of wildlife rehabilitators were assumed to include repeated contact with a small number of bats, involving hand feeding (especially for little brown bats), medical management of injuries, with a contact duration of weeks to months. We assumed that most rehabilitators typically dedicate an enclosed room for rehabilitation activities, with facilities that may range from a shed or garage to a purpose-built structure. 
The definition of enclosed space includes attics of various sizes and dimensions that may result in variation in airflow among sites. Wildlife control operators typically do not enter enclosed spaces during the summer season, so as not to disturb bats who may be rearing pups. For bats within a home's living space, a wildlife control operator may catch a bat for release. We assumed that activity in enclosed space may be greater than 1 hour, and bats in these spaces may be a mixture of stationary (roosting) and in flight. Typical activities near bats but in an unenclosed space include a wildlife control operator working to exclude bats from a home (that is, installing an excluder device near soffits or eaves after young bats are flying and not likely to be trapped inside when their mothers go out to forage).

\section{References Cited}

Adams-Hosking, C., McBride, M.F., Baxter, G., Burgman, M., de Villiers, D., Kavanagh, R., Lawler, I., Lunney, D., Melzer, A., Menkhorst, P., Molsher, R., Moore, B.D., Phalen, D., Rhodes, J.R., Todd, C., Whisson, D., and McAlpine, C.A., 2016, Use of expert knowledge to elicit population trends for the koala (Phascolarctos cinereus): Diversity \& Distributions, v. 22, no. 3, p. 249-262 accessed May 19, 2020, at https://doi.org/10.1111/ddi.12400.

Ayyub, B.M., 2001, Elicitation of expert opinions for uncertainty and risks: Boca Raton, Fla., USA, CRC, 302 p.

Burgman, M.A., McBride, M., Ashton, R., Speirs-Bridge, A., Flander, L., Wintle, B., Fidler, F., Rumpff, L., and Twardy, C., 2011, Expert status and performance: PLoS One, v. 6, no. 7, p. e22998, accessed May 19, 2020, at https://doi.org/10.1371/journal.pone.0022998.

Fazey, I., Fazey, J.A., Salisbury, J.G., Lindenmayer, D.B., and Dovers, S., 2006, The nature and role of experiential knowledge for environmental conservation: Environmental Conservation, v. 33, no. 1, p. 1-10, accessed May 19, 2020, at https://doi.org/10.1017/S037689290600275X.

Hanea, A., McBride, M., Burgman, M., Wintle, B., Fidler, F., Flander, L., Twardy, C., Manning, B., and Mascaro, S., 2017, Investigate Discuss Estimate Aggregate for structured expert judgement: International Journal of Forecasting, v. 33, no. 1, p. 267-279, accessed May 19, 2020, at https:// doi.org/10.1016/j.ijforecast.2016.02.008.

Hemming, V., Walshe, T.V., Hanea, A.M., Fidler, F., and Burgman, M.A., 2018, Eliciting improved quantitative judgements using the IDEA protocol - A case study in natural resource management: PLoS One, v. 13, no. 6, p. e0198468, accessed May 19, 2020, at https://doi.org/10.1371/journal. pone. 0198468 .
Hogarth, R.M., 1978, A note on aggregating opinions: Organizational Behavior and Human Performance, v. 21, no. 1, p. 40-46, accessed May 19, 2020, at https://doi. org/10.1016/0030-5073(78)90037-5.

Martin, T.G., Burgman, M.A., Fidler, F., Kuhnert, P.M., LowChoy, S., McBride, M., and Mengersen, K., 2012, Eliciting expert knowledge in conservation science: Conservation Biology, v. 26, no. 1, p. 29-38, accessed May 19, 2020, at https://doi.org/10.1111/j.1523-1739.2011.01806.x.

Morgan, M.G., 2014, Use (and abuse) of expert elicitation in support of decision making for public policy: Proceedings of the National Academy of Sciences of the United States of America, v. 111, no. 20, p. 7176-7184, accessed May 19, 2020, at https://doi.org/10.1073/pnas.1319946111.

O’Hagan, A., 2019, Expert knowledge elicitation-subjective but scientific: The American Statistician, v. 73 sup. 1, p. 69-81.

O’Hagan, A., Buck, C.E., Daneshkhah, A., Eiser, J.R., Garthwaite, P.H., Jenkinson, D.J., Oakley, J.E., and Rakow, T., 2006, Uncertain judgements-Eliciting experts' probabilities: West Sussex, UK, John Wiley \& Sons, accessed May 19, 2020, at https://doi.org/10.1002/0470033312

Runge, M.C., Converse, S.J., and Lyons, J.E., 2011, Which uncertainty? Using expert elicitation and expected value of information to design an adaptive program: Biological Conservation, v. 144, no. 4, p. 1214-1223, accessed May 19, 2020, at https://doi.org/10.1016/j.biocon.2010.12.020.

Speirs-Bridge, A., Fidler, F., McBride, M.F., Flander, L., Cumming, G., and Burgman, M.A., 2010, Reducing overconfidence in the interval judgments of experts: Risk Analysis, v. 30, no. 3, p. 512-523, accessed May 19, 2020, at https:// doi.org/10.1111/j.1539-6924.2009.01337.x.

Sutherland, W.J., and Burgman, M., 2015, Policy adviceUse experts wisely: Nature, v. 526, no. 7573, p. 317-318, accessed May 19, 2020, at https://doi.org/10.1038/526317a. 


\section{Appendix 2. Expert Elicitation Results}

Results of responses to Questions 1-13 are shown in illustrations. 
$\boldsymbol{A}$
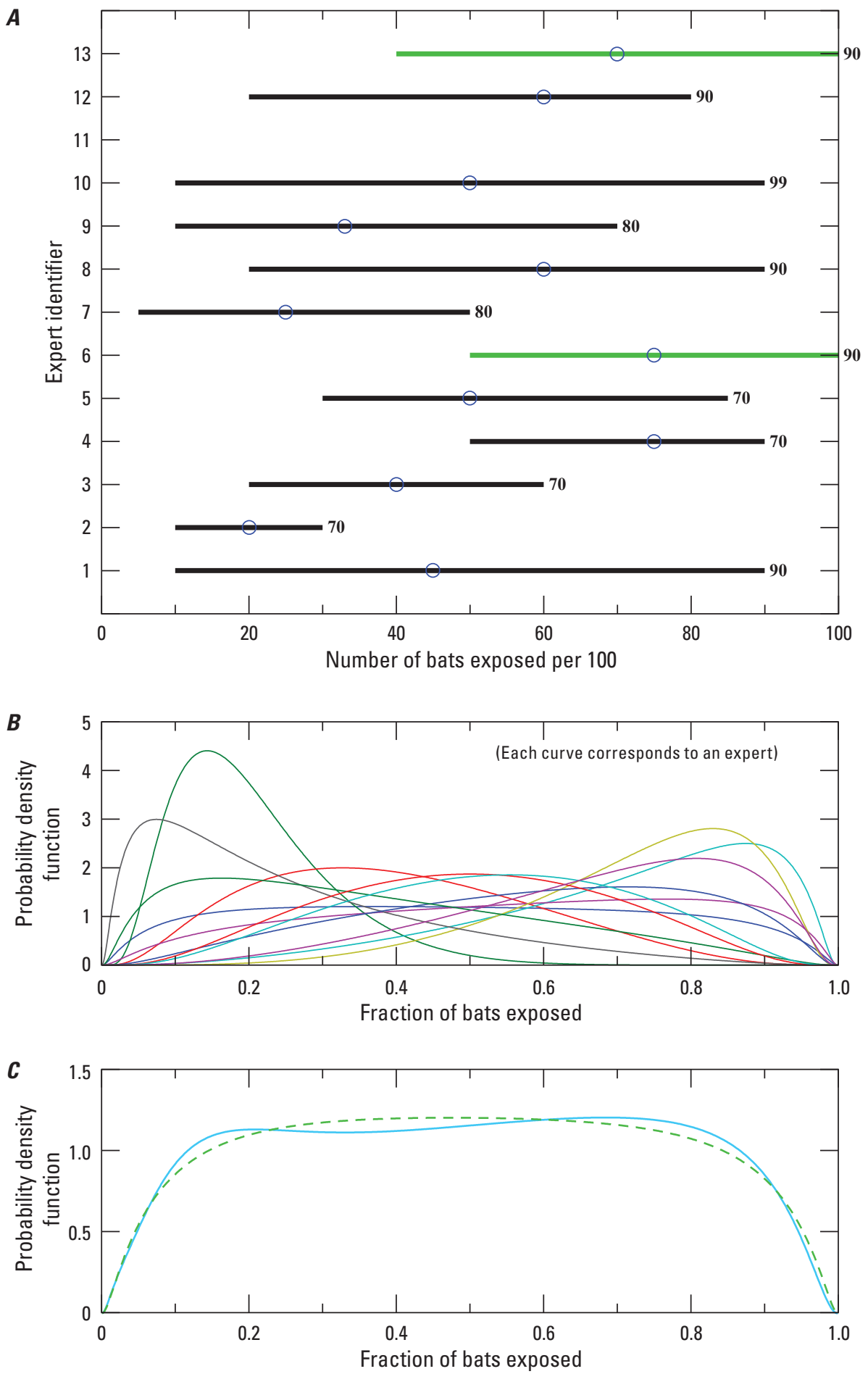

\section{EXPLANATION}

Two-sided confidence interval One-sided confidence interval

○ Median estimate

90 Confidence level

\section{EXPLANATION}

Average across experts

Fitted aggregate distribution across experts

Figure 2.1. Expert panel responses to Question 1-number of bats exposed to virus by a SARS-CoV-2-positive scientist handling bats. $A$, Four-point-elicitation responses from the individual experts, $B$, fitted probability distributions for individual experts, and $C$, average and fitted distributions across experts. The aggregate distribution has a median of 49.7 bats and an 80 -percent confidence interval of $(15.3,84.3)$. 
$\boldsymbol{A}$

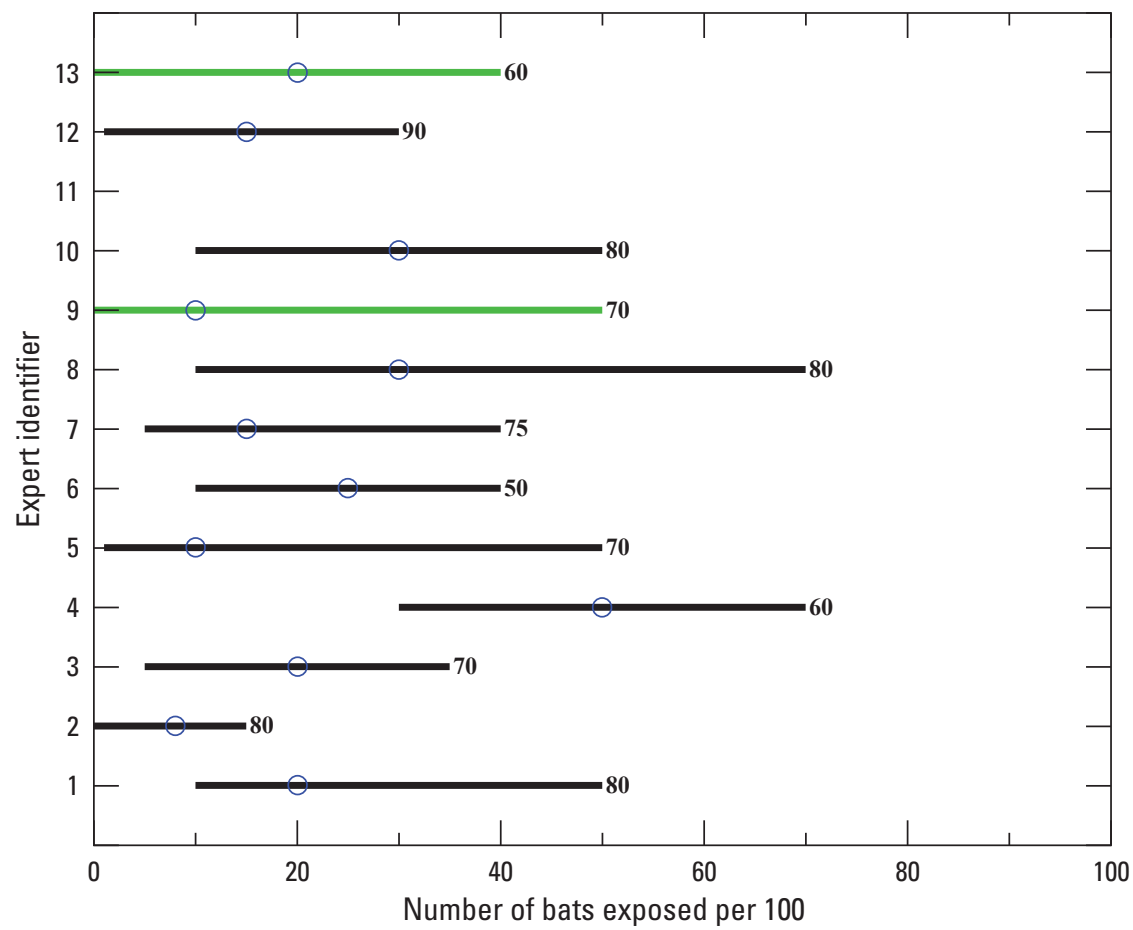

EXPLANATION

Two-sided confidence interval

One-sided confidence interval

O Median estimate

90 Confidence level
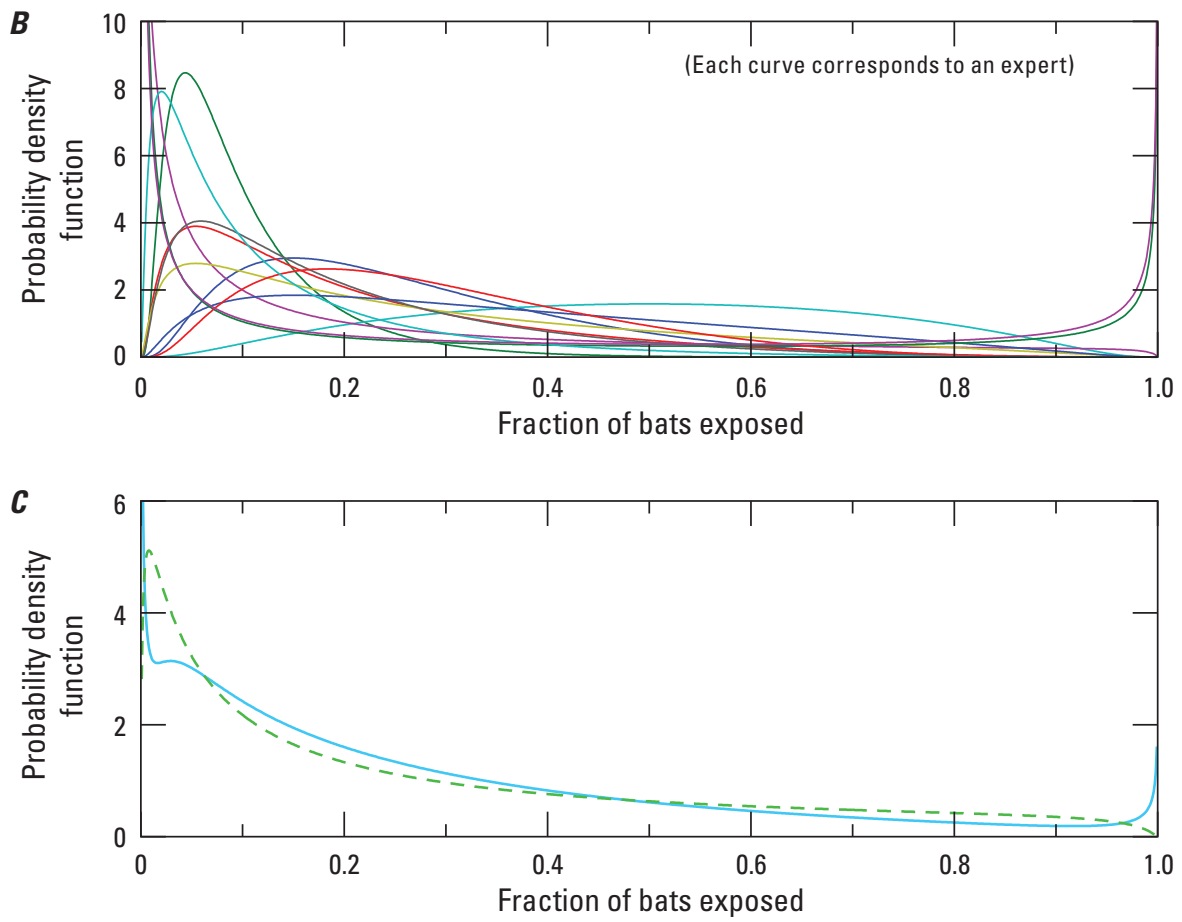

EXPLANATION

Average across experts

- - - - Fitted aggregate distribution across experts

Figure 2.2. Expert panel responses to Question 2-number of bats exposed to virus by a SARS-CoV-2-positive scientist in an enclosed space within 6 feet of bats. $A$, Four-point-elicitation responses from the individual experts, $B$, fitted probability distributions for individual experts, and $C$, average and fitted distributions across experts. The aggregate distribution has a median of 19.4 bats and an 80-percent confidence interval of $(2.2,72.4)$. 
$\boldsymbol{A}$
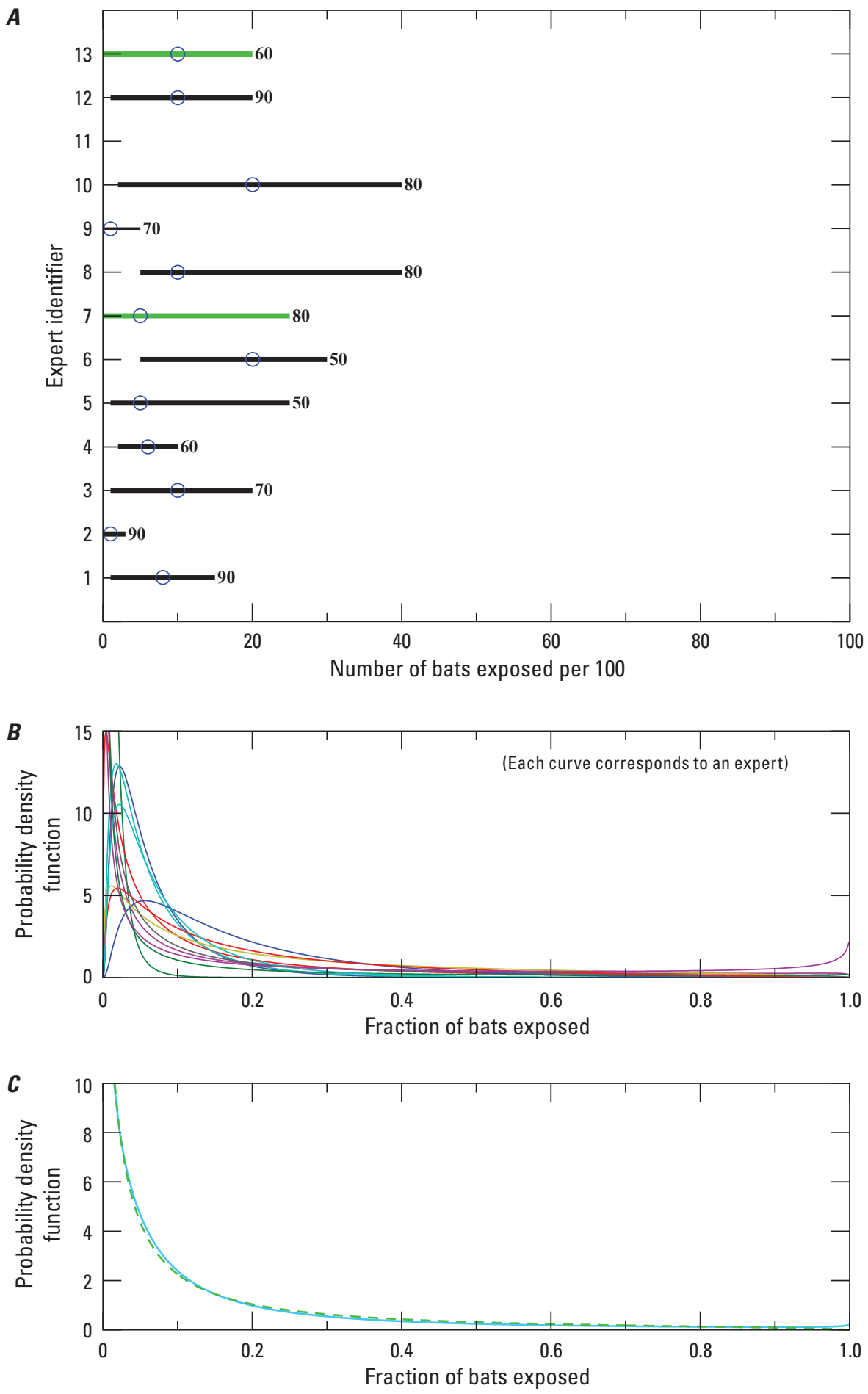

\section{EXPLANATION}

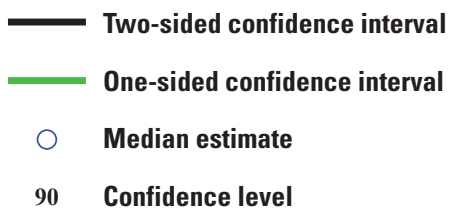

90

\section{EXPLANATION}

Average across experts

Fitted aggregate distribution across experts

Figure 2.3. Expert panel responses to Question 3-number of bats exposed to virus by a SARS-CoV-2-positive scientist in an unenclosed space within 6 feet of bats. $A$, Four-point-elicitation responses from the individual experts, $B$, fitted probability distributions for individual experts, and $C$, average and fitted distributions across experts. The aggregate distribution has a median of 6.4 bats and an 80 -percent confidence interval of $(0.6,43.8)$. 
A
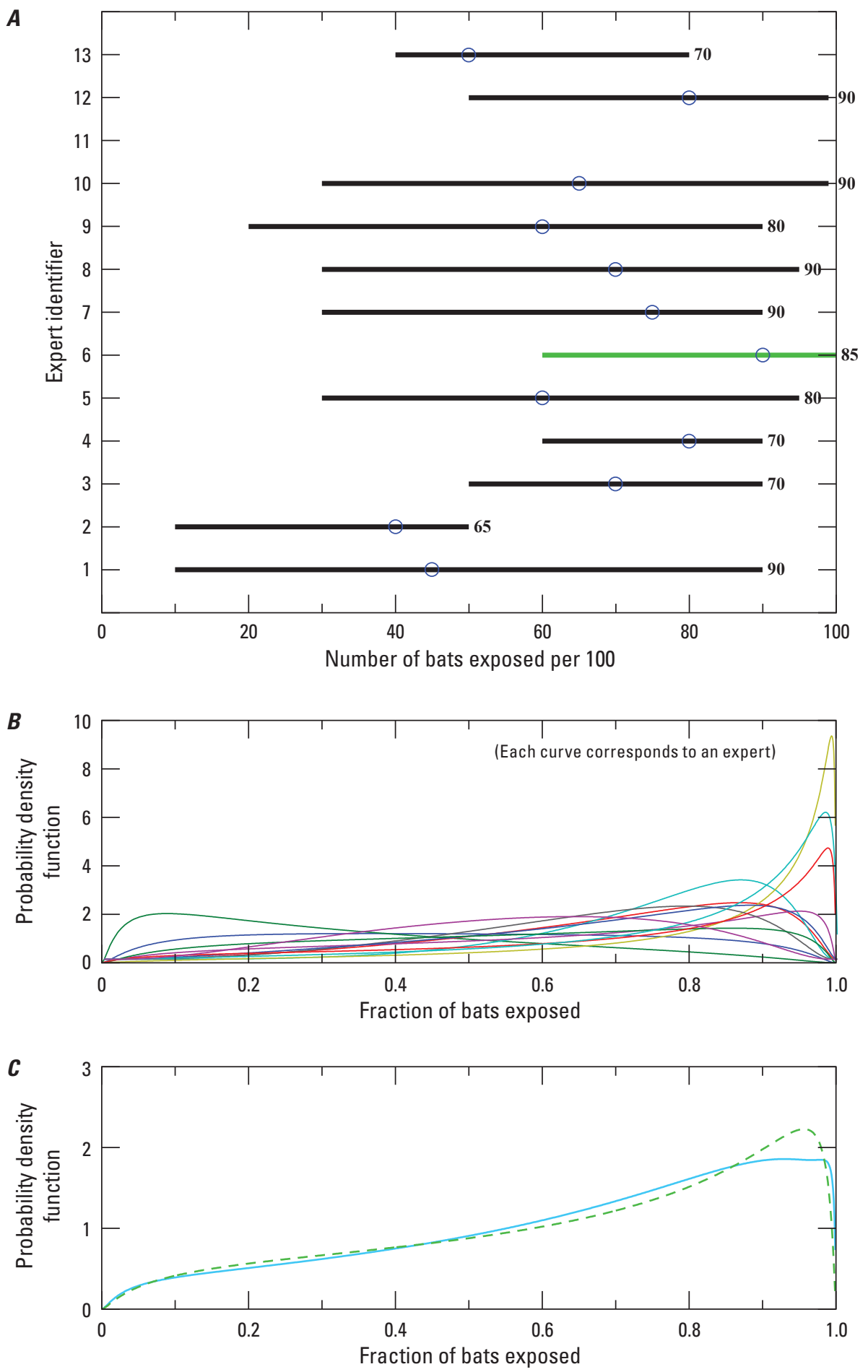

EXPLANATION

Two-sided confidence interval

One-sided confidence interval

- Median estimate

90 Confidence level
EXPLANATION

Average across experts

Fitted aggregate distribution across experts

Figure 2.4. Expert panel responses to Question 4-number of bats exposed to virus by a SARS-CoV-2-positive wildlife rehabilitator handling bats. $A$, Four-point-elicitation responses from the individual experts, $B$, fitted probability distributions for individual experts, and $C$, average and fitted distributions across experts. The aggregate distribution has a median of 70.4 bats and an 80-percent confidence interval of $(24.4,94.6)$. 
A
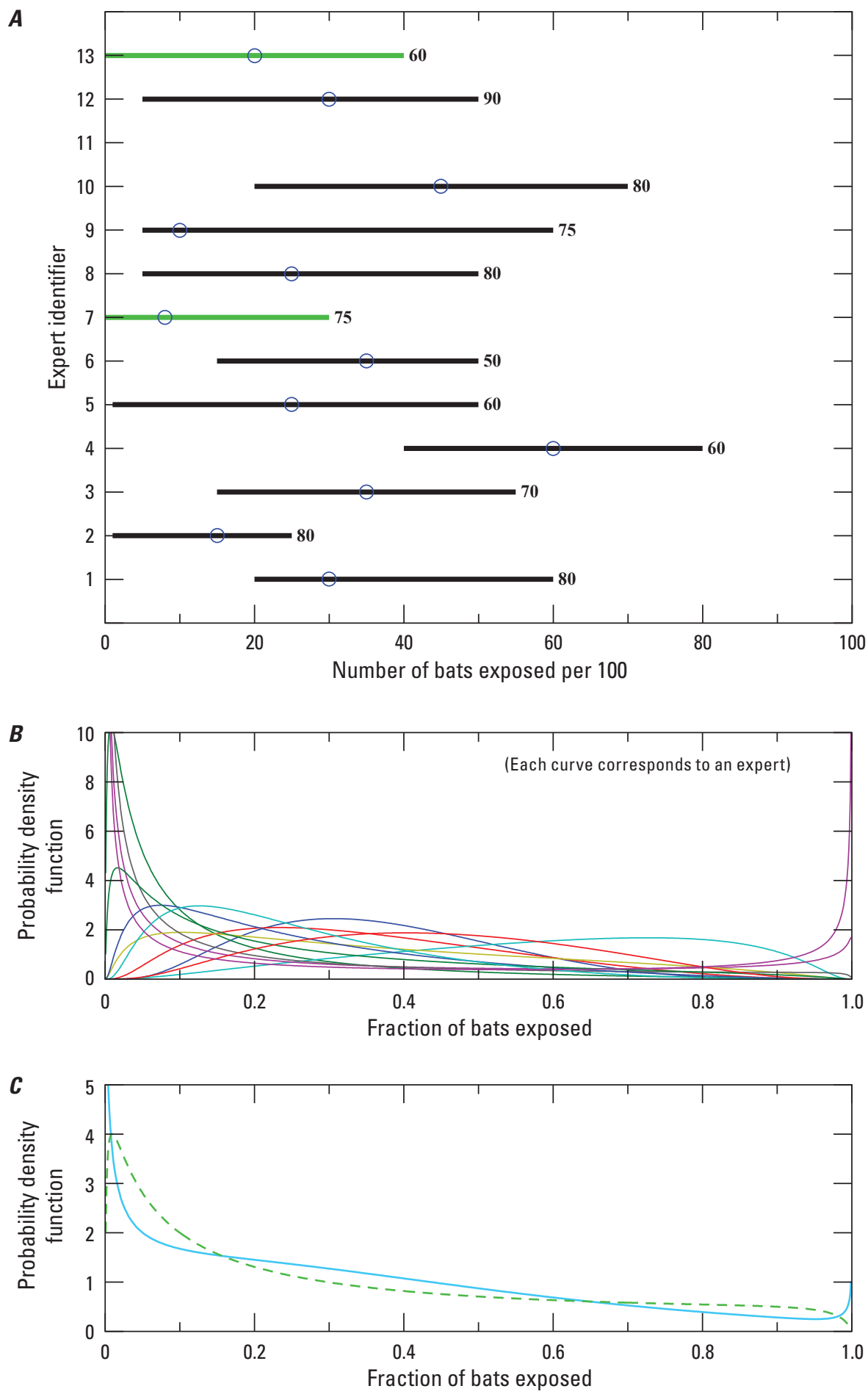

\section{EXPLANATION}

Two-sided confidence interval

One-sided confidence interval

Median estimate

90 Confidence level
EXPLANATION

Average across experts

Fitted aggregate distribution across experts

Figure 2.5. Expert panel responses to Question 5-number of bats exposed to virus by a SARS-CoV-2-positive wildlife rehabilitator within 6 feet of bats. $A$, Four-point-elicitation responses from the individual experts, $B$, fitted probability distributions for individual experts, and $C$, average and fitted distributions across experts. The aggregate distribution has a median of 24.3 bats and an 80-percent confidence interval of $(2.8,78.4)$. 
A

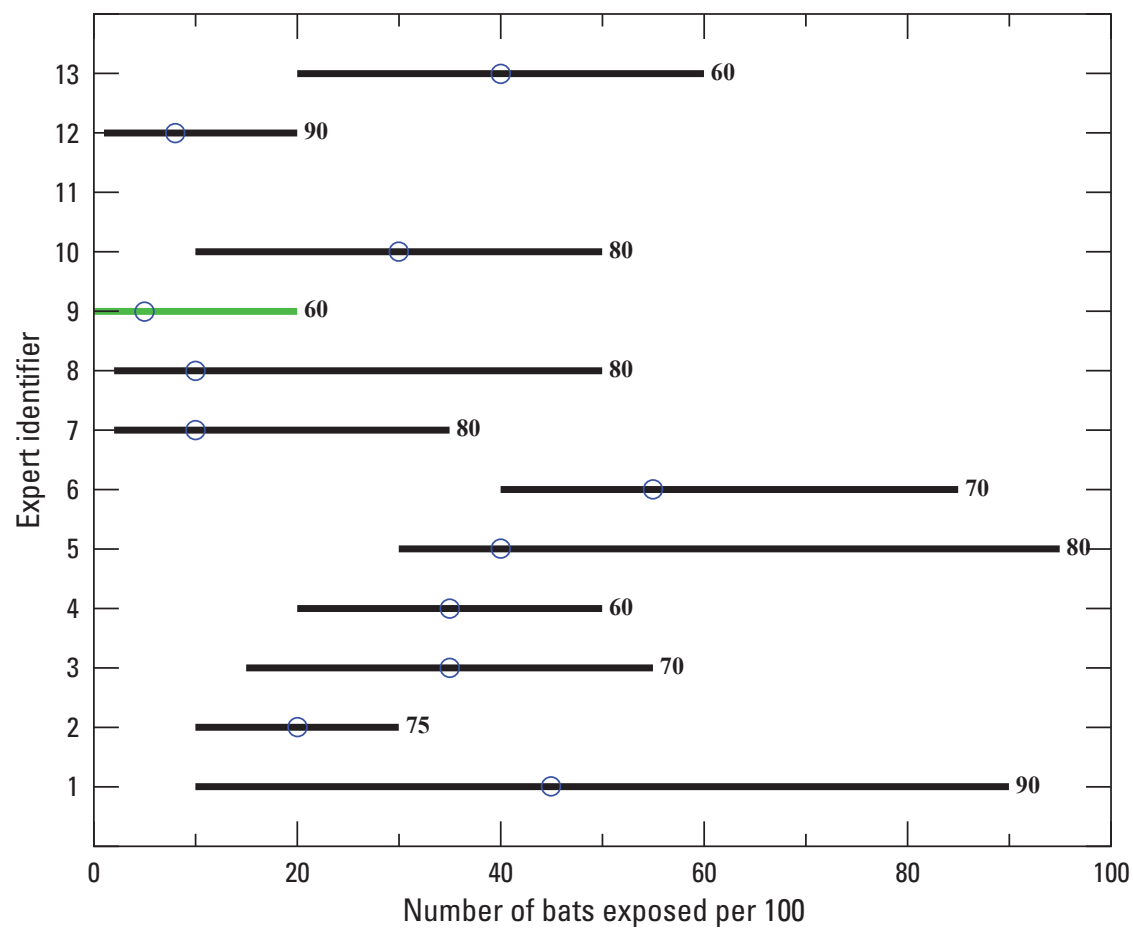

EXPLANATION

Two-sided confidence interval

One-sided confidence interval

O Median estimate

90 Confidence level
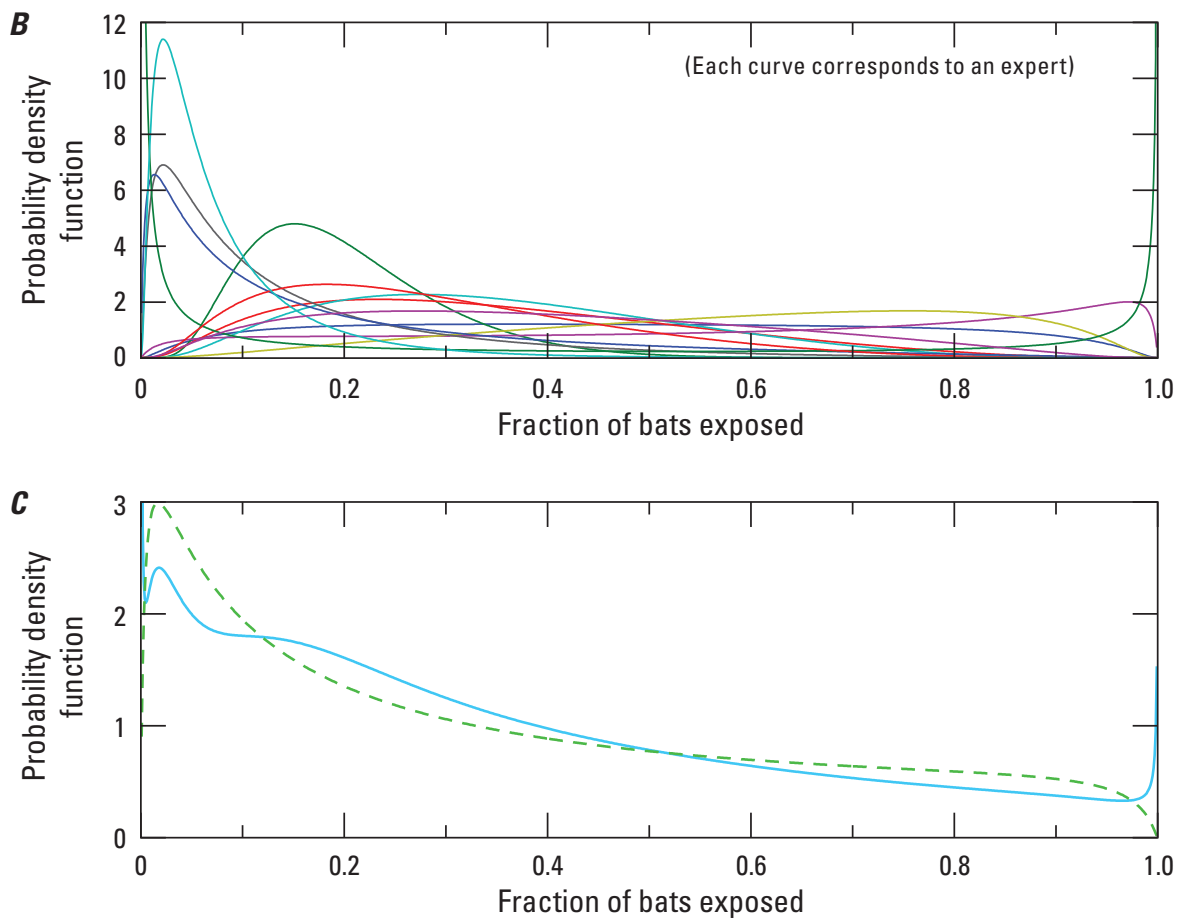

EXPLANATION

Average across experts

- - - - Fitted aggregate distribution across experts

Figure 2.6. Expert panel responses to Question 6-number of bats exposed to virus by a SARS-CoV-2-positive wildlife control operator handling bats. $A$, Four-point-elicitation responses from the individual experts, $B$, fitted probability distributions for individual experts, and $C$, average and fitted distributions across experts. The aggregate distribution has a median of 27.7 bats and an 80-percent confidence interval of $(3.7,79.2)$. 
$\boldsymbol{A}$

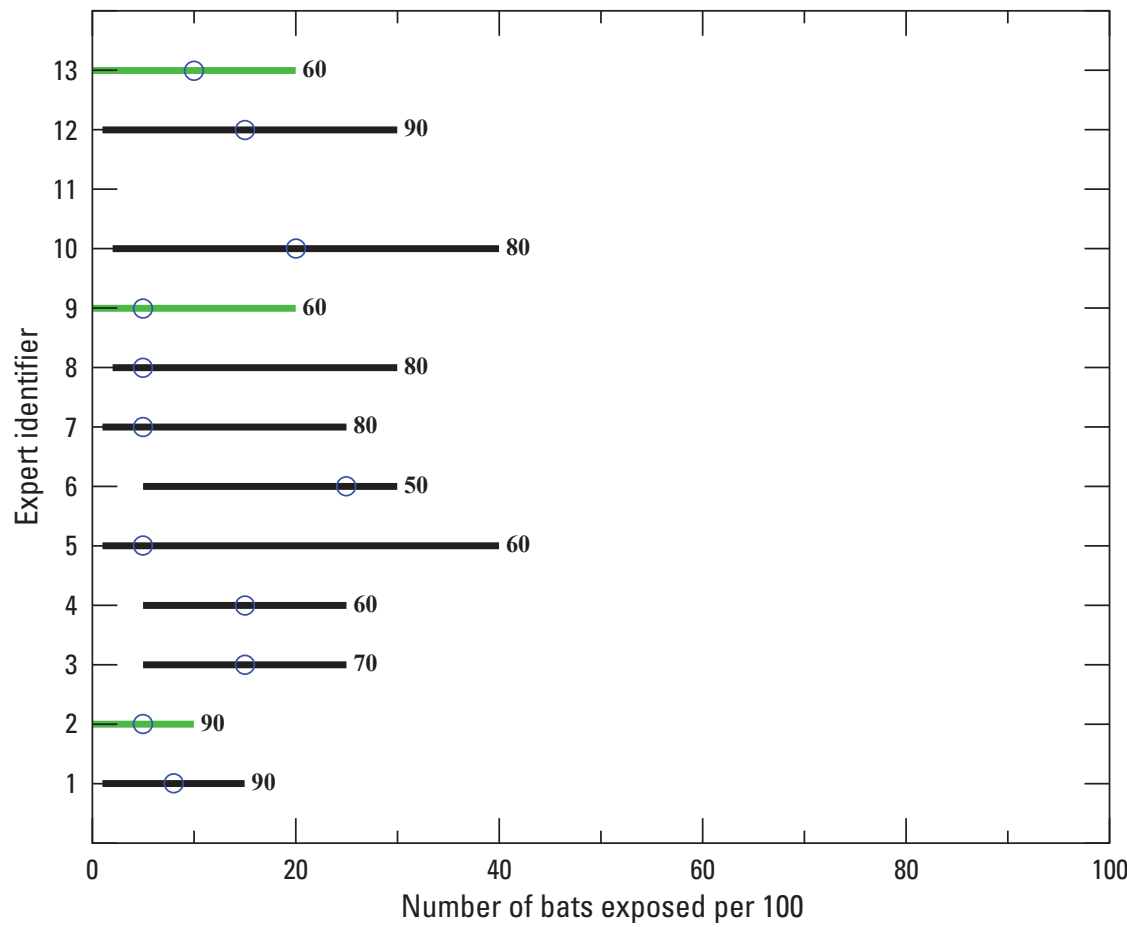

EXPLANATION

Two-sided confidence interval

One-sided confidence interval

$\bigcirc \quad$ Median estimate

90

Confidence level
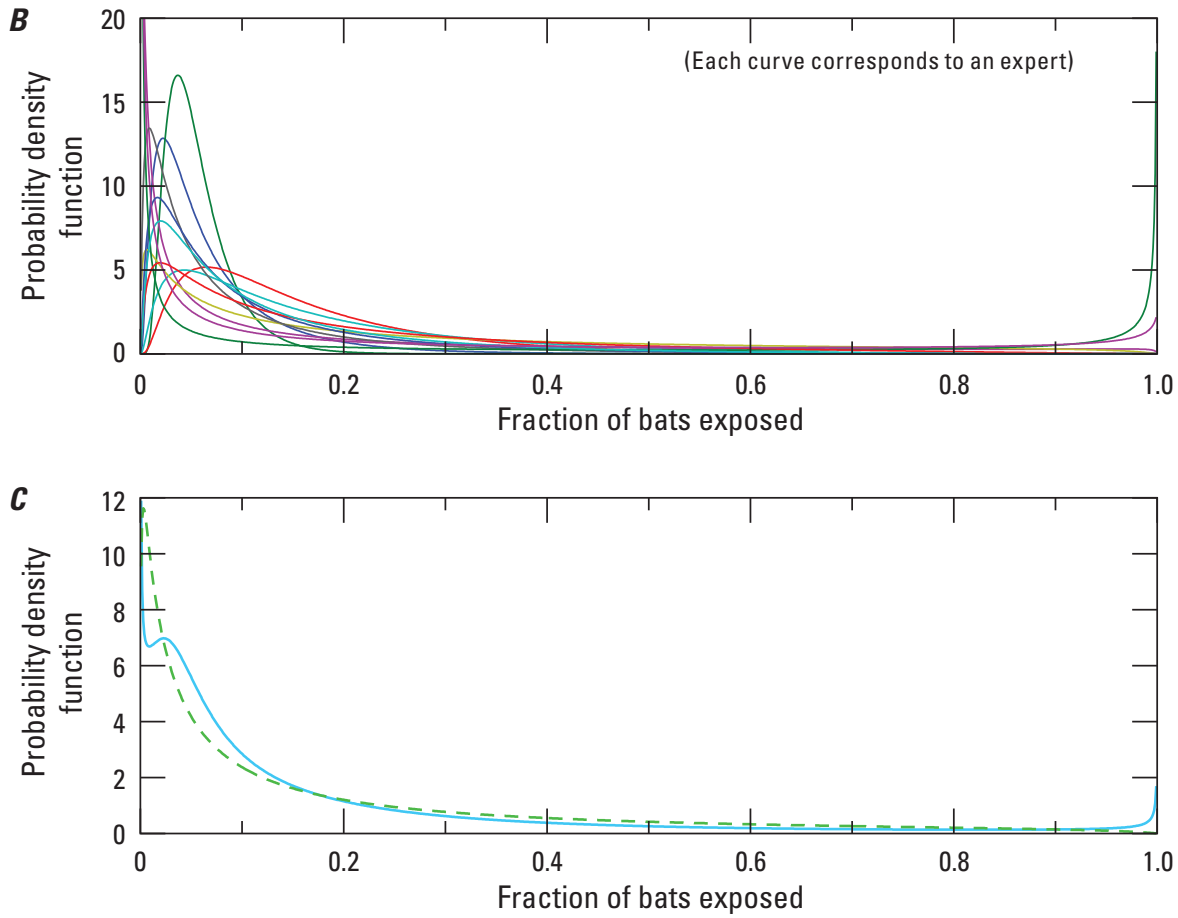

EXPLANATION

Average across experts

- - - Fitted aggregate distribution across experts

Figure 2.7. Expert panel responses to Question 7-number of bats exposed to virus by a SARS-CoV-2-positive wildlife control operator within 6 feet of bats. $A$, Four-point-elicitation responses from the individual experts, $B$, fitted probability distributions for individual experts, and $C$, average and fitted distributions across experts. The aggregate distribution has a median of 9.6 bats and an 80 -percent confidence interval of $(1.0,53.9)$. 
A
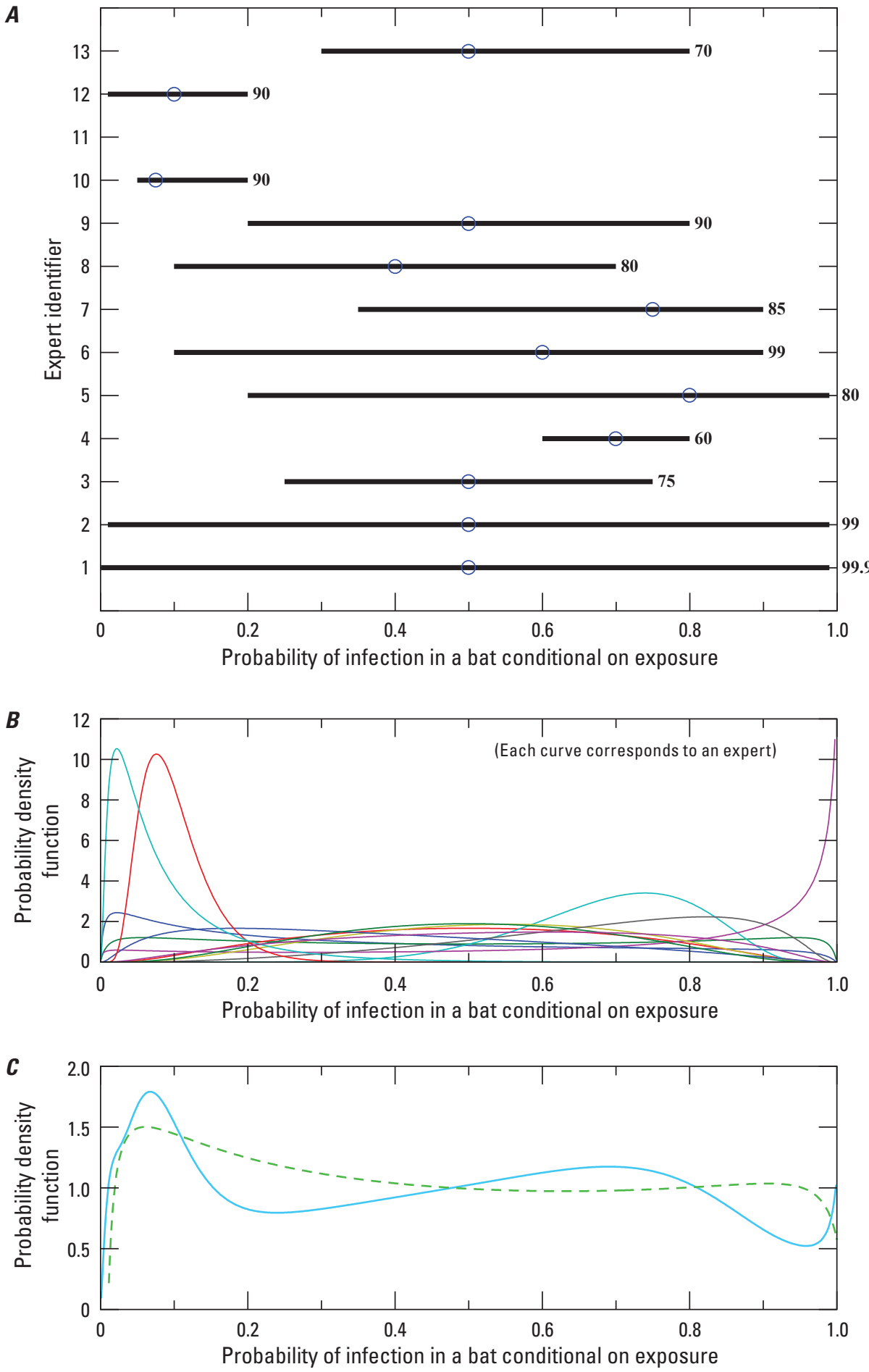

\section{EXPLANATION}

Two-sided confidence interval

$\bigcirc \quad$ Median estimate

90 
$\boldsymbol{A}$

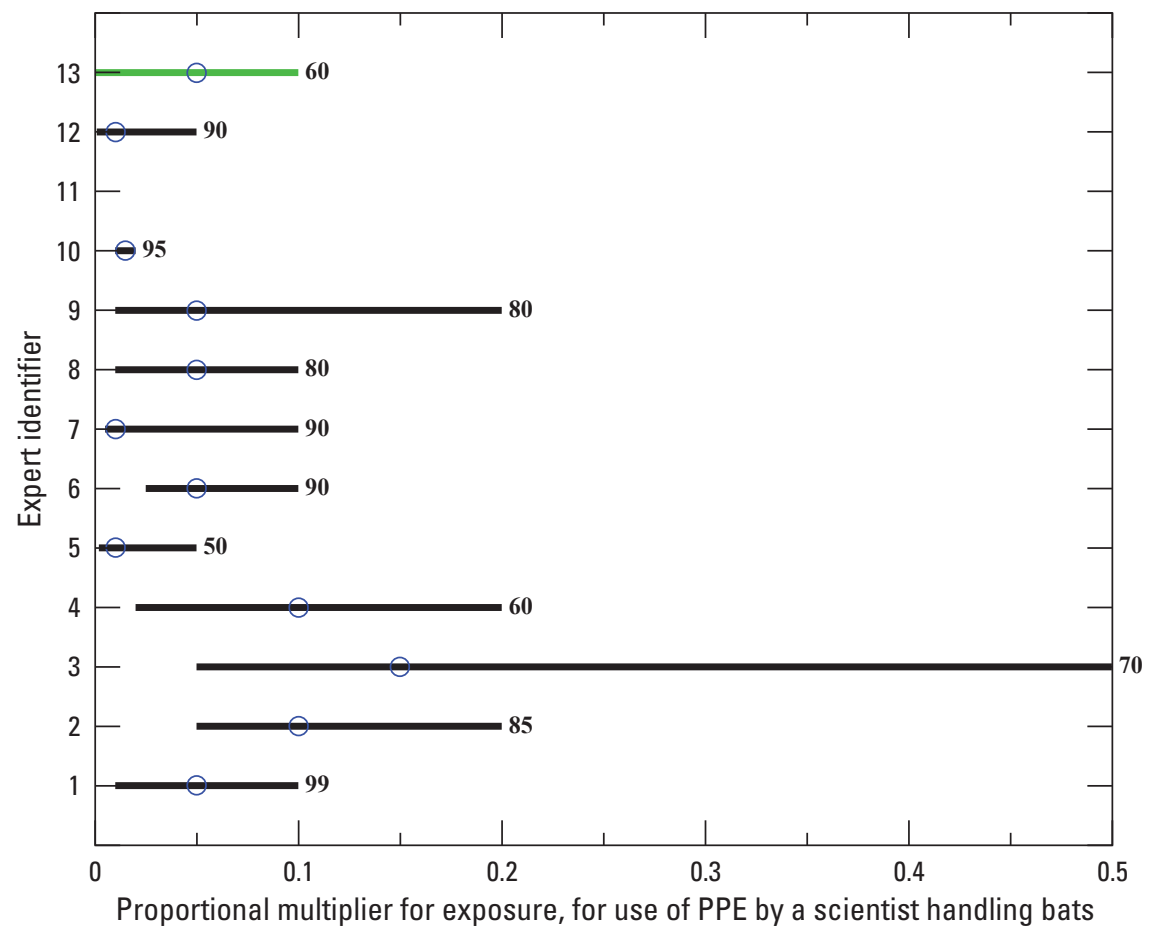

\section{EXPLANATION}

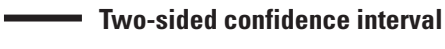

One-sided confidence interval

Median estimate

90 Confidence level
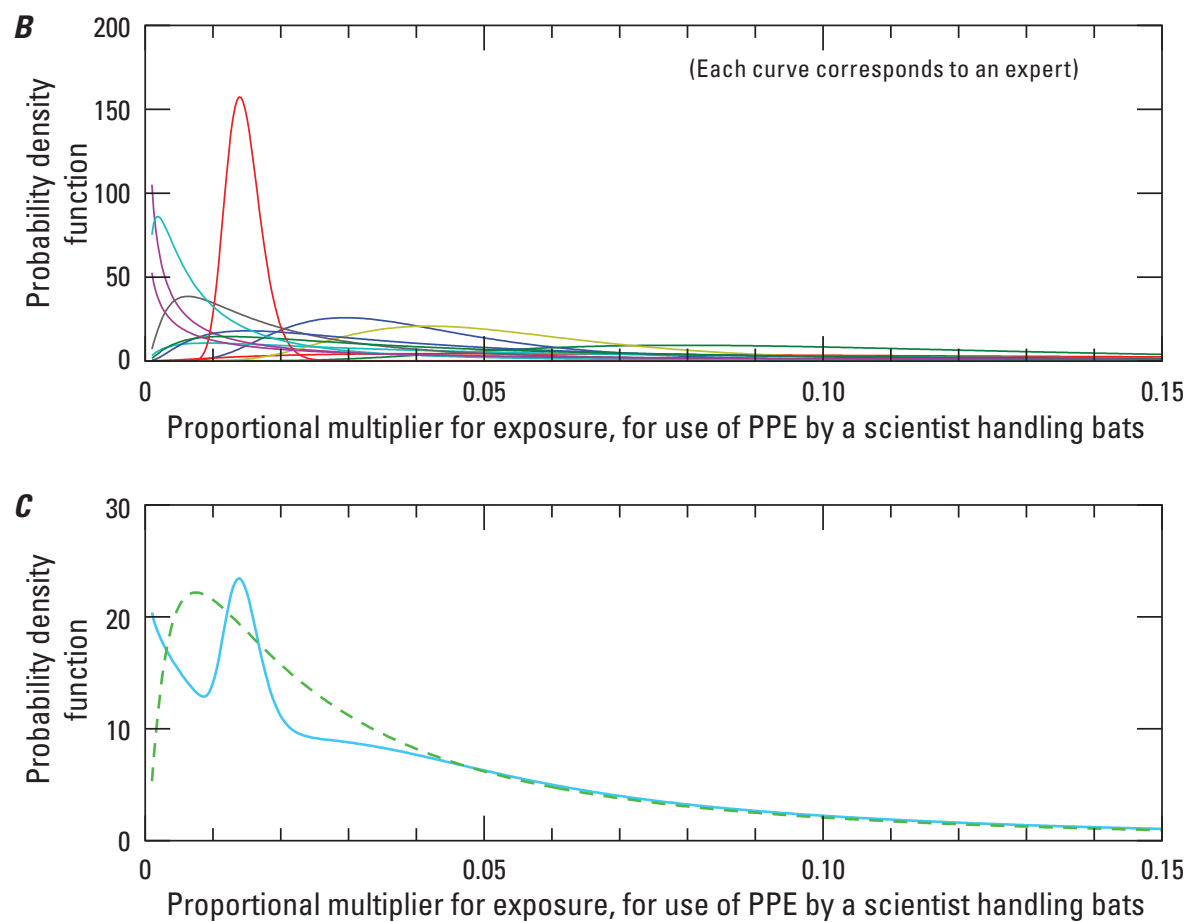

EXPLANATION

Average across experts

- - - - Fitted aggregate distribution across experts

Figure 2.9. Expert panel responses to Question 9-multiplier for exposure, when using personal protective equipment, for a research scientist handling bats. $A$, Four-point-elicitation responses from the individual experts, $B$, fitted probability distributions for individual experts, and $C$, average and fitted distributions across experts. The aggregate distribution has a median of 0.031 and an 80 -percent confidence interval of $(0.007,0.141)$. PPE, personal protective equipment. 
$\boldsymbol{A}$

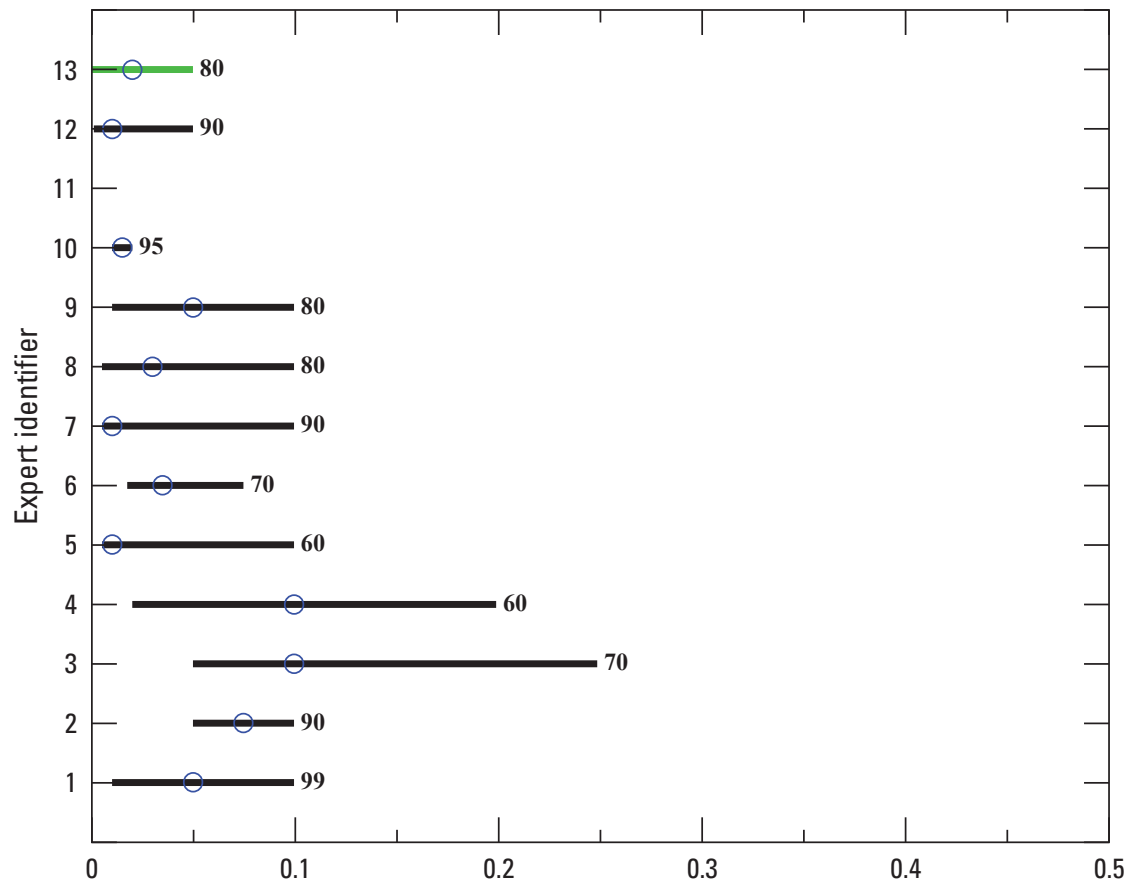

EXPLANATION

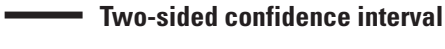

One-sided confidence interval

O Median estimate

$90 \quad$ Confidence level

Proportional multiplier for exposure, for use of PPE by a scientist in an enclosed space

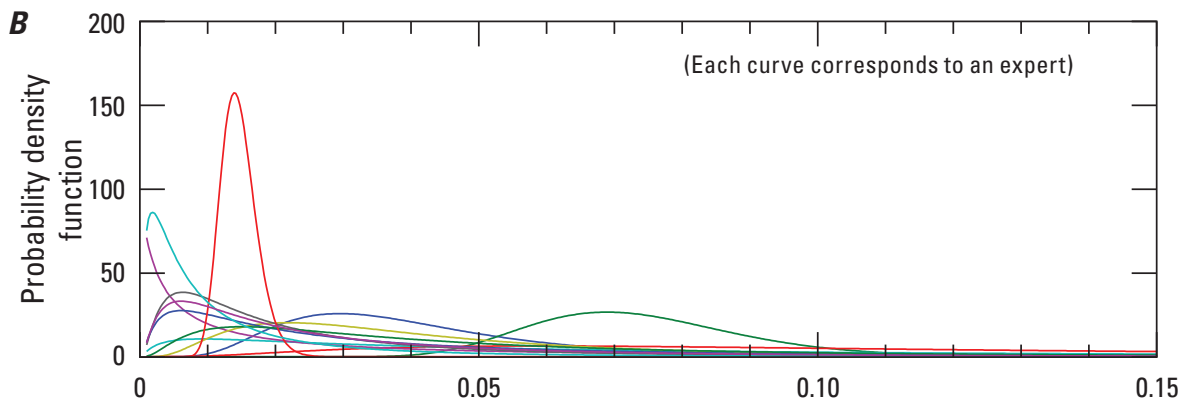

Proportional multiplier for exposure, for use of PPE by a scientist in an enclosed space

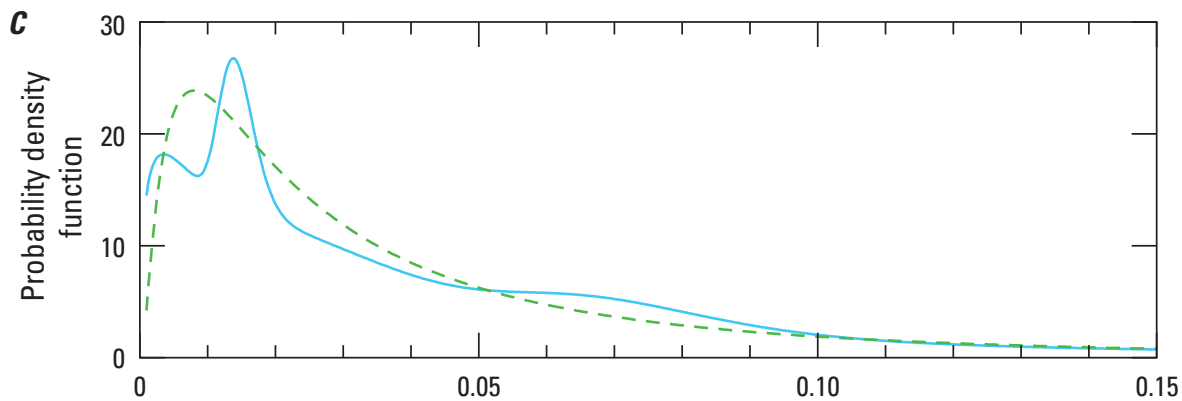

EXPLANATION

Average across experts

- - - - Fitted aggregate distribution across experts

Proportional multiplier for exposure, for use of PPE by a scientist in an enclosed space

Figure 2.10. Expert panel responses to Question 10-multiplier for exposure, when using personal protective equipment, for a research scientist in an enclosed space within 6 feet of bats. $A$, Four-point-elicitation responses from the individual experts, $B$, fitted probability distributions for individual experts, and $C$, average and fitted distributions across experts. The aggregate distribution has a median of 0.028 and an 80 -percent confidence interval of $(0.007,0.117)$. PPE, personal protective equipment. 
$\boldsymbol{A}$
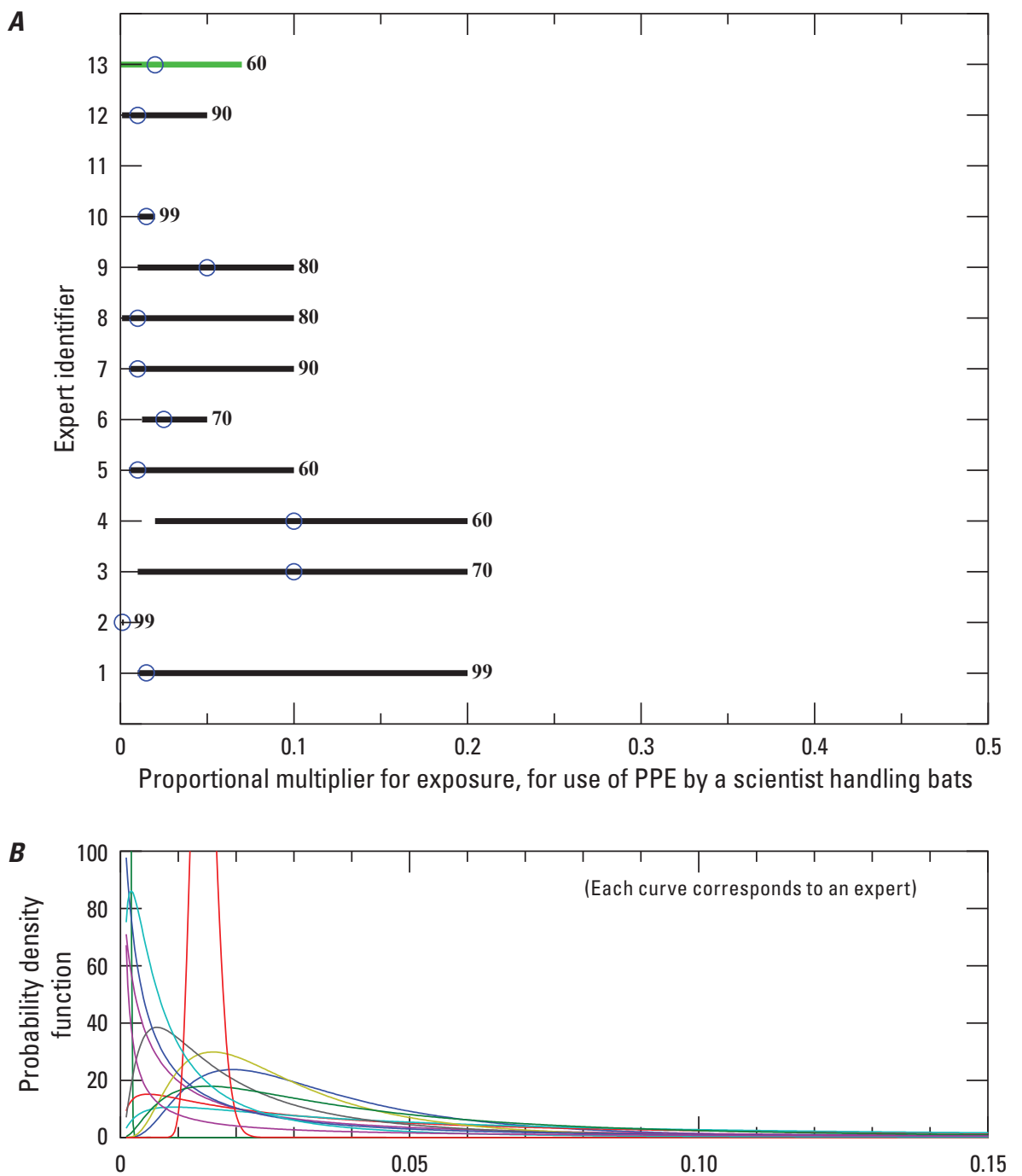

Proportional multiplier for exposure, for use of PPE by a scientist in an unenclosed space

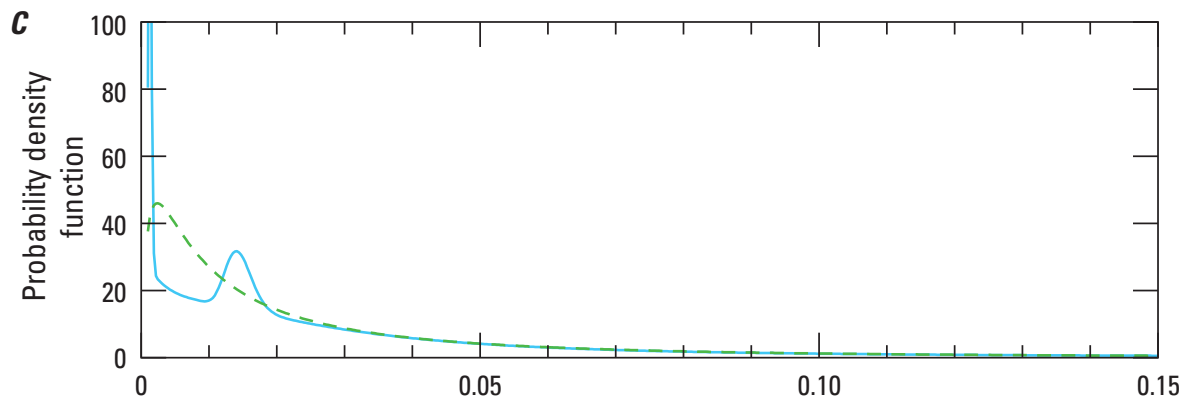

Proportional multiplier for exposure, for use of PPE by a scientist in an unenclosed space

\section{EXPLANATION}

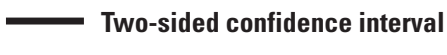

One-sided confidence interval

Median estimate

90 Confidence level

\section{EXPLANATION}

Average across experts

Fitted aggregate distribution across experts

Figure 2.11. Expert panel responses to Question 11-multiplier for exposure, when using personal protective equipment, for a research scientist in an unenclosed space within 6 feet of bats. $A$, Four-point-elicitation responses from the individual experts, $B$, fitted probability distributions for individual experts, and $C$, average and fitted distributions across experts. The aggregate distribution has a median of 0.016 and an 80 -percent confidence interval of $(0.003,0.096)$. PPE, personal protective equipment. 
A

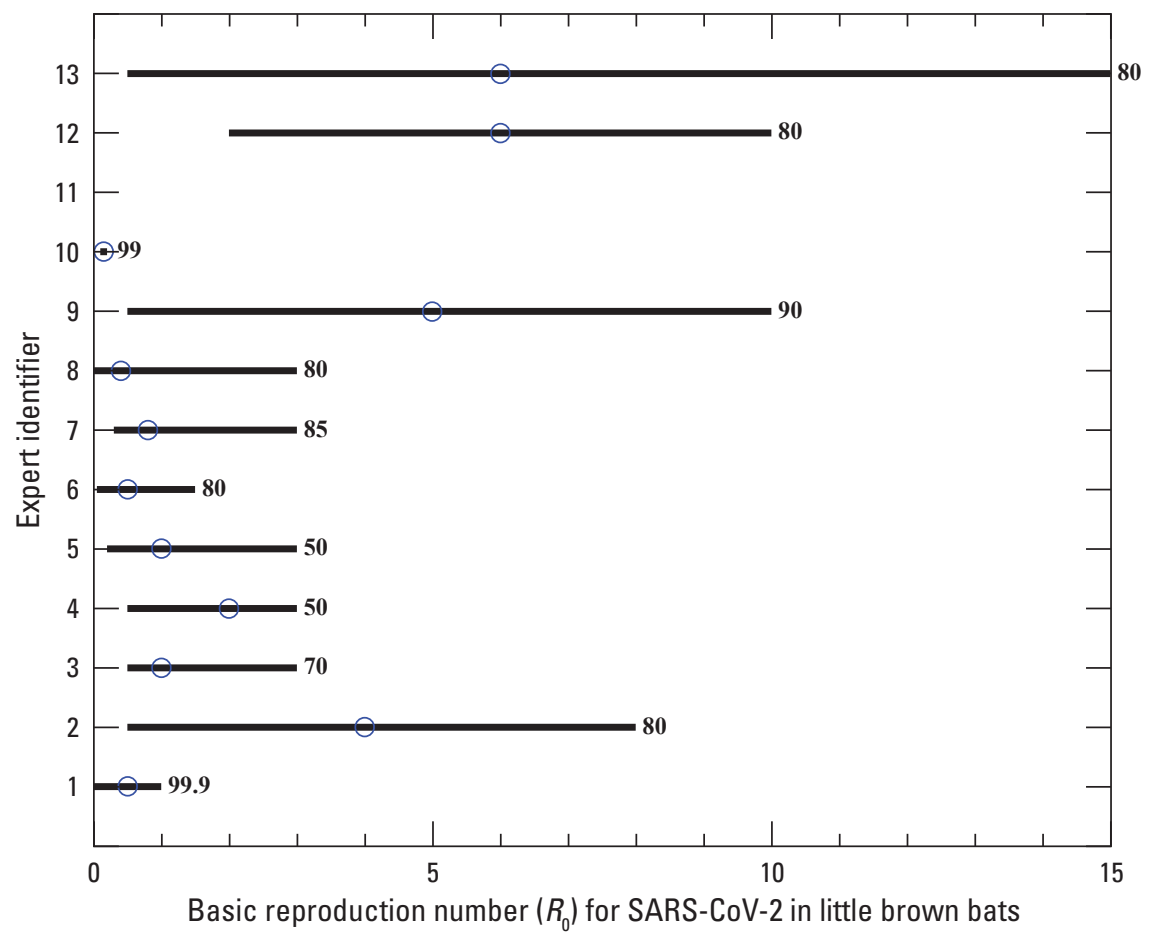

EXPLANATION

Two-sided confidence interval

O Median estimate

90

Confidence level
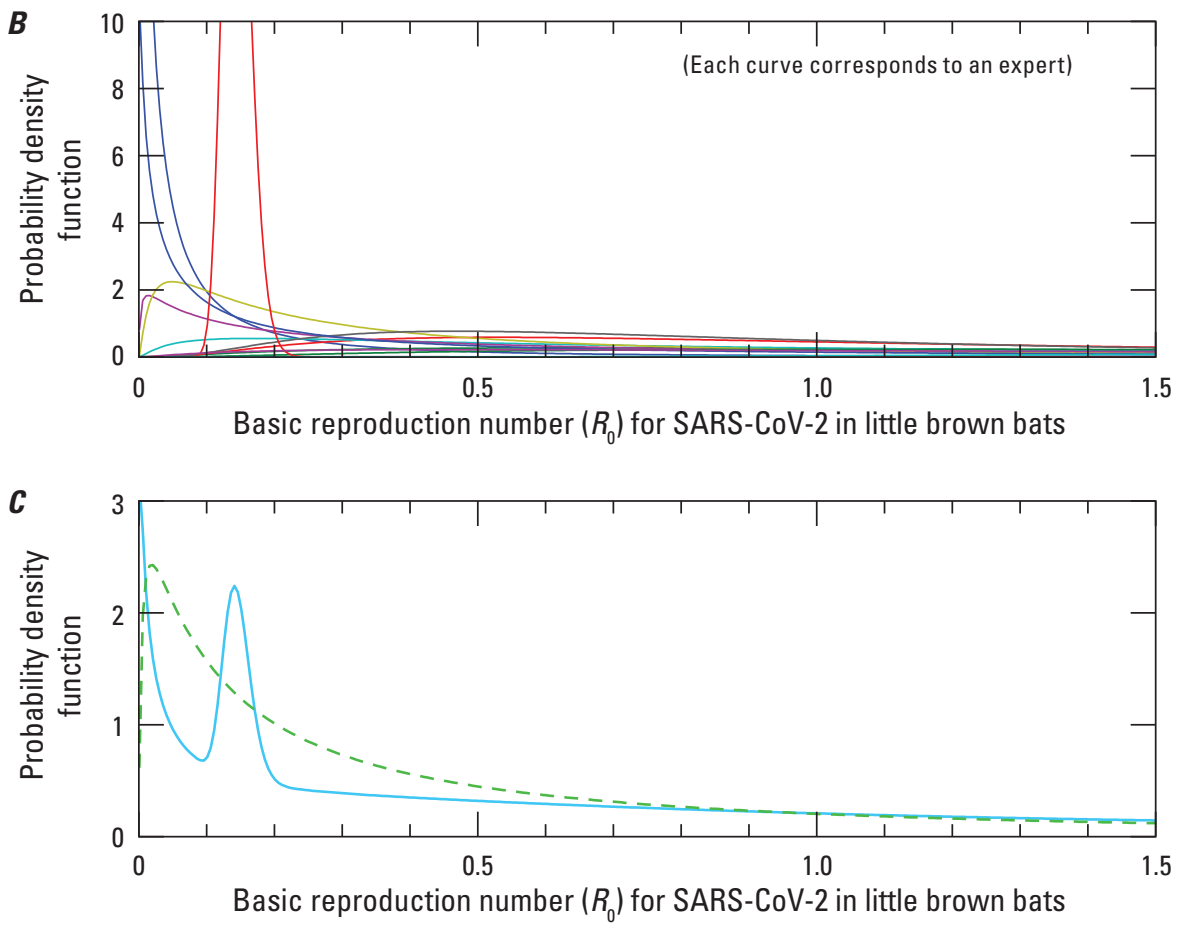

EXPLANATION

Average across experts

- - - - Fitted aggregate distribution across experts

Figure 2.12. Expert panel responses to Question 13-SARS-CoV-2 $R_{0}$ in little brown bats. $A$, Four-point-elicitation responses from the individual experts, $B$, fitted probability distributions for individual experts, and $C$, average and fitted distributions across experts. The aggregate distribution has a median of 0.45 and an 80 -percent confidence interval of $(0.05,4.38)$. In the aggregate distribution, the probability that $R_{0}$ is greater than 1.0 is 0.326 . 

For additional information, contact:

Director, Patuxent Wildlife Research Center U.S. Geological Survey

12100 Beech Forest Road

Laurel, MD 20708

or visit our website at: https://www.usgs.gov/centers/pwrc

Publishing support provided by the West Trenton Publishing Service Center 
